\title{
Applications physico-chimiques des plasmas d'arc
}

\author{
P. Fauchais \\ Laboratoire Céramiques Nouvelles, LA 320, Université de Limoges, \\ U.E.R. des Sciences, 87060 Limoges Cedex, France
}

(Reçu le 3 avril 1984, révisé le 3 juillet, accepté le 13 septembre 1984)

\begin{abstract}
Résumé. - Depuis quelques années l'intérêt industriel pour les plasmas thermiques va grandissant et le nombre d'applications développées est sans cesse croissant. Il nous a donc semblé qu'il convenait, au travers de la littérature, de faire le point de la question non seulement sur les applications développées ou potentiellement développables, mais aussi et surtout sur nos connaissances actuelles des phénomènes mis en jeu ou impliqués. C'est donc pourquoi nous nous proposons de voir successivement :

- Les conditions d'équilibre des plasmas thermiques ainsi que leurs propriétés thermodynamiques et de transport.

- La génération des plasmas avec les problèmes des électrodes, de la colonne de plasma et les conséquences qui en découlent pour la réalisation des générateurs à cathodes chaudes ou froides ainsi que les problèmes liés à leur utilisation (temps de séjour, homogénéité du traitement, introduction des produits à traiter, mélange) avec la réalisation de fours à plasma destinés à améliorer le temps de séjour ou les transferts thermiques.

- La modélisation des plasmas avec l'écoulement réactif ou non et le mélange d'un gaz avec le plasma, les transferts de quantité de mouvement et de chaleur et les points essentiels qui se dégagent de cette modélisation.

- Les applications actuelles et potentielles de ces plasmas thermiques pour le chauffage, la production de gaz réducteurs, les réactions chimiques, la fusion et la purification en plasma, la vaporisation, la métallurgie extractive, la projection.
\end{abstract}

\begin{abstract}
Since a few years the interest for the use of thermal plasmas in industry is growing and the number of applications developed is increasing. In this paper we try, through the recent literature on the subject, to make an overview of the state of the art, of the developed applications and also of our actual knowledge of the phenomena involved. Thus we will see successively :

- The equilibrium conditions in thermal plasmas and the related thermodynamic and transport properties.

- The plasma generation with the electrodes phenomena, the arc column and the consequences for the practical realization of plasma torches either with hot or cold cathodes, together with the problems related to their use (residence time, homogenity of the treatment, introduction of the products to be treated, mixing...) problems partly solved by the development of plasma furnaces allowing to increase the residence time and the heat transfer between plasma and solid materials.

- The modelization of a reactive or non reactive plasma flow, of the mixing of a cold gas with the plasma, of the momentum, heat and mass transfer between plasma and particles underlying of course the main points arising from these models.

- The actual and potential applications of these thermal plasma for heating, production of reducing, nitriding, oxidizing gases, chemical reactions, melting, remelting and purification of solid materials, vaporization, extractive metallurgy, coal gazeification, spraying...
\end{abstract}

\section{Les propriétés thermiques et de transport des plasmas thermiques.}

1.1 DÉfINITION. - Nous allons restreindre notre étude au cas des plasmas produits par arc électrique à la pression atmosphérique. Dans de tels milieux, macroscopiquement électriquement neutres et constitués d'atomes, de molécules et d'ions dans l'état fondamental et dans des états excités ainsi que d'électrons, le nombre de particule est tel (supérieur à $10^{16}$ particules $/ \mathrm{cm}^{3}$ ) que les très nombreuses collisions entre les particules font que l'énergie cinétique moyenne de toutes les particules (les lourdes et les électrons) est pratiquement identique. En effet, si on ne considère que les collisions élastiques, le transfert d'énergie entre deux particules de masses respectives 
$m$ et $M$ est caractérisé [1] par le coefficient

$$
K=\frac{2 m M}{(m+M)^{2}}
$$

et l'on voit donc que si le transfert est important pour deux particules de même masse, il est très faible si $m \ll M$ comme c'est le cas des électrons par rapport aux atomes, molécules et ions (par exemple pour l'argon $K \sim 1 / 35000$ ) et qu'il faut beaucoup de collisions pour « thermaliser " les électrons. Si l'on définit alors la température comme correspondant à l'énergie cinétique moyenne des particules $\left(\frac{3}{2} k T=\frac{1}{2} \overline{m v^{2}}\right)$ pour une distribution Maxwellienne des vitesses, on peut établir facilement [2] que pour un gaz mono-atomique l'écart à l'équilibre entre la température des électrons $T_{\mathrm{e}}$ et celle des particules lourdes $T_{\mathrm{h}}$ est défini par la relation :

$$
\frac{T_{\mathrm{e}}-T_{\mathrm{h}}}{T_{\mathrm{e}}}=\frac{\pi M}{24 m}\left(\frac{\lambda_{\mathrm{e}} e E}{k T_{\mathrm{e}}}\right)^{2}
$$

où $\lambda_{\mathrm{e}}$ est le libre parcours moyen des électrons et $E$ le champ électrique. Par exemple pour un plasma d'argon atmosphérique avec $E=13 \mathrm{~V} / \mathrm{cm}, \lambda_{\mathrm{e}}=$ $3 \times 10^{-4} \mathrm{~cm}, M / m=7 \times 10^{4}$ et $T_{\mathrm{e}}=10^{4} \mathrm{~K}$, la déviation entre $T_{\mathrm{e}}$ et $T_{\mathrm{h}}$ est inférieure à $1 \%$.

D'une manière générale, nous admettons donc que, dans les plasmas thermiques à la pression atmosphérique où la densité des électrons $n_{\mathrm{e}}$ est supérieure à $10^{16} \mathrm{e} / \mathrm{cm}^{3}$, l'équilibre est réalisé. Cependant cet état d'équilibre n'est pas nécessairement réalisé soit dans le panache de tels jets où $n_{\mathrm{e}}<10^{16}$ $\mathrm{e} / \mathrm{cm}^{3}$ [3], soit lorsque les gradients de concentration ou de température sont suffisamment élevés pour que la diffusion des électrons soit rapide comparativement à celle des particules lourdes (aux frontières du jet de plasma ou au voisinage des électrodes ou de parois refroidies) [4], soit lors de l'injection d'un gaz froid dans le plasma [5].

\subsection{LES PROPRIÉTÉS THERMODYNAMIQUES DE TRANS-} PORT.

1.2.1 L'équilibre. - Au cour du jet de plasma avec des densités d'électrons supérieures à $10^{16} \mathrm{e} / \mathrm{cm}^{3}$, on peut supposer que les cinétiques de réaction sont ultrarapides par rapport aux cinétiques physiques ou mécaniques (vitesses d'écoulement, diffusion, ...) et il est donc possible de déterminer les propriétés du plasma à partir de l'équilibre thermodynamique : composition, enthalpie $h$, viscosité $\mu$, conductivité électrique $\sigma$, conductivité thermique $\kappa$.

Nous ne reviendrons pas sur le calcul des propriétés thermodynamiques (composition, enthalpie) [6 à 15] et de transport $(\mu, \sigma, \kappa)$ [16 à 31] des plasmas à l'équilibre dans la mesure où ceux-ci sont maintenant bien classiques. Signalons simplement une bibliographie récente [32] des propriétés de transport à l'équilibre ainsi qu'une étude [33] de l'influence des potentiels d'interaction sur les propriétés de transport.
Il convient cependant de souligner un des points importants de ces propriétés, à savoir leur non-linéarité avec la température. Par exemple pour la chaleur spécifique, ceci se traduit par des zones de température correspondant à l'existence de pics, pics dûs aux réactions chimiques telles que la dissociation et les ionisations successives (comme on peut le voir sur la figure 1 pour les quatre gaz plasmagènes les plus utilisés) et qui correspondent à des variations brutales de l'enthalpie.

Dans le cadre du concept de la qualité de la chaleur produite, il est très important de comparer l'enthalpie d'un plasma et celle d'une flamme. En effet, celle d'une flamme est conditionnée par la nature des réactifs et la température de combustion; elle est donc limitée. En revanche, pour un plasma, l'énergie est fournie par une source extérieure (la puissance électrique $V \times I$ peut varier de un à deux ordres de grandeur) ainsi que le débit de gaz : on peut donc atteindre des énergies très élevées $\left(10^{8} \mathrm{~kJ} / \mathrm{kg}\right)$ qui vont servir, si l'on considère le plasma uniquement comme un système thermique, à dissocier et ioniser le gaz et le porter finalement à des températures moyennes comprises entre $3000 \mathrm{~K}$ et $15000 \mathrm{~K}$.

Westinghouse [34] a montré qu'un réchauffeur à plasma à air où l'enthalpie disponible peut atteindre $9298 \mathrm{~kJ} / \mathrm{kg}$ permet de récupérer beaucoup plus d'énergie qu'un réchauffeur à flamme air-gaz naturel où l'enthalpie ne dépasse pas $3161 \mathrm{~kJ} / \mathrm{kg}$. Par exemple,

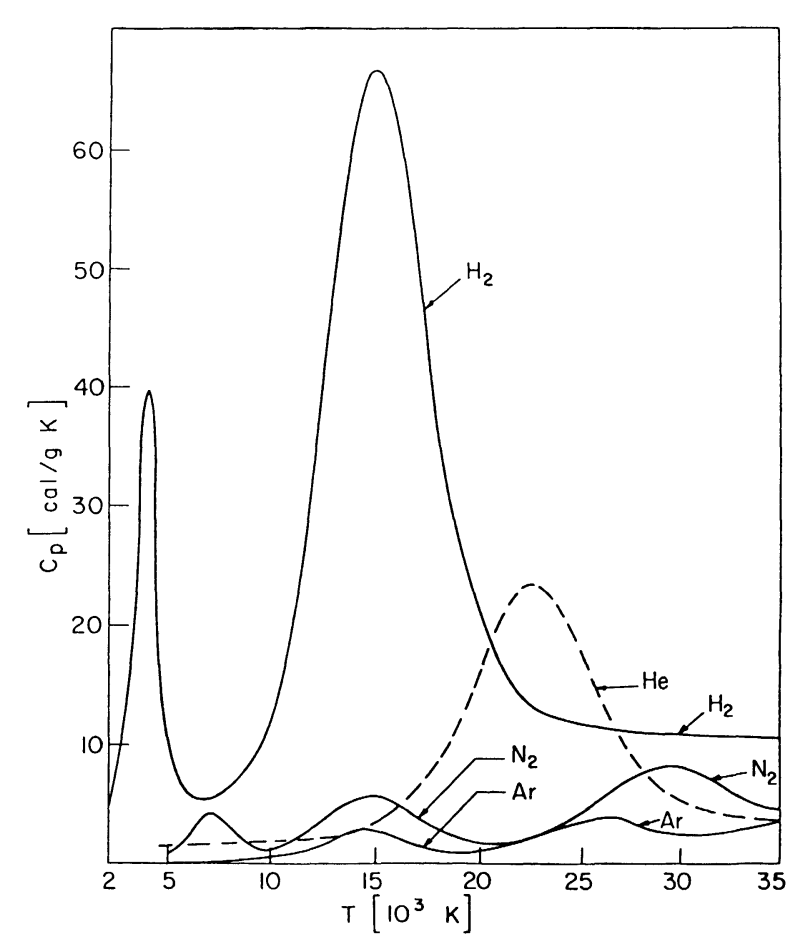

Fig. 1. - Evolution en fonction de la température et à la pression atmosphérique de la chaleur spécifique de l'argon, de l'azote, de l'hydrogène et de l'hélium $[14,15]$.

[Evolution with temperature, at atmospheric pressure, of the specific heat of argon, nitrogen, hydrogen and helium plasmas at equilibrium $[14,15]$. 
pour réchauffer des lingots de fer ou des brames à $1400^{\circ} \mathrm{C}$, le réchauffeur à combustion ne permet de récupérer que $10 \%$ de l'énergie fournie, alors que le réchauffeur à plasma permet de récupérer $70 \%$ de l'énergie introduite (cf. Fig. 2).

Il est également intéressant de souligner la valeur relative de la conductivité thermique des différents gaz les plus couramment utilisés dans les plasmas thermiques : argon, azote, hydrogène, conductivité thermique représentée $[35,76]$ sur la figure 3 . Il est clair, sur cette figure, que l'hydrogène est meilleur conducteur que l'argon. C'est, entre autres, pour cette raison que l'on utilise l'hydrogène pur ou en mélange dans les divers réacteurs plasma. La croissance importante de conductivité thermique observée pour l'hydrogène à $3500 \mathrm{~K}$ et pour l'azote à $6000 \mathrm{~K}$ est due aux phénomènes de dissociation (conductivité thermique réactive). Pour des gaz tels que $\mathrm{CO}_{2}, \mathrm{CH}_{4}, \mathrm{H}_{2} \mathrm{O}$, les températures de dissociation étant plus basses, la conductivité thermique est plus importante à basse température, ce qui facilite les transferts.

1.2.2 Les modèles à deux températures. - Une première approche du non-équilibre est développée depuis quelques années avec les modèles à deux températures : particules lourdes à la température $T_{\mathrm{h}}$, électrons à la température $T_{\mathrm{e}}$. Tous les modèles développés jusqu'alors supposent que les distributions de vitesses des particules restent maxwelliennes, que les niveaux d'excitation suivent une loi de Boltzmann due aux collisions avec les électrons pour les états électroniques (ce qui revient à négliger le déséquilibre de population observé pour les niveaux de résonance et leurs voisins) et aux collisions avec les lourds pour les distributions de rotation, vibration et translation, que l'équilibre d'ionisation n'est pas perturbé.

1.2.2.1 Propriétés thermodynamiques. - Un écart de température entre $T_{\mathrm{e}}$ et $T_{\mathrm{h}}$ entraîne une perturbation de la loi d'action de masse notamment pour les réactions d'ionization. A la suite des premiers travaux de Potapov [37], la concentration des particules chargées dans un plasma, déterminée à partir de l'équation de bilan des réactions d'ionization-recombinaison, peut être obtenue [37 à 45] enl écrivant l'équation de Saha de l'équilibre mais où le rapport des densités des ions et des particules est élevé à la puissance $1 / \theta$ avec $\theta=T_{\mathrm{e}} / T_{\mathrm{h}}$ et où la température d'ionization est prise égale à $T_{\mathrm{e}}$, les fonctions de partition électroniques étant calculées également à $T_{\mathrm{e}}$ [45]. Toutes les autres lois d'action de masse ne faisant pas intervenir les particules chargées sont conservées, la température $T_{\mathrm{h}}$ étant prise pour toutes les fonctions de partition non électronique et la température $T_{\mathrm{e}}$ pour les fonctions de partition électronique et naturellement les équations de conservation ainsi que de neutralité électrique sont conservées. Pour une valeur donnée des paramètres $\theta$ les calculs procèdent alors de la même façon qu'à l'équilibre [7 à 9]. La figure 4, tirée de [43] représente par exemple, pour différentes valeurs de $\theta$, la variation

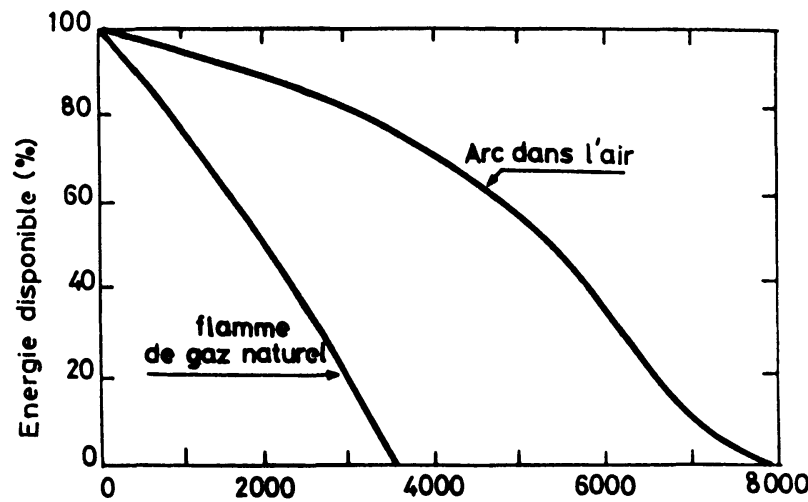

Fig. 2. - Evolution avec la température de chauffage d'une brame (en degrés Fahrenheit) du pourcentage de l'énergie disponible dans une flamme de gaz naturel et dans un plasma d'air [34].

[Evolution with the heating temperature (degrees Fahrenheit) of the percentage of available energy in a methane flame and in an air plasma at atmospheric pressure [34].]

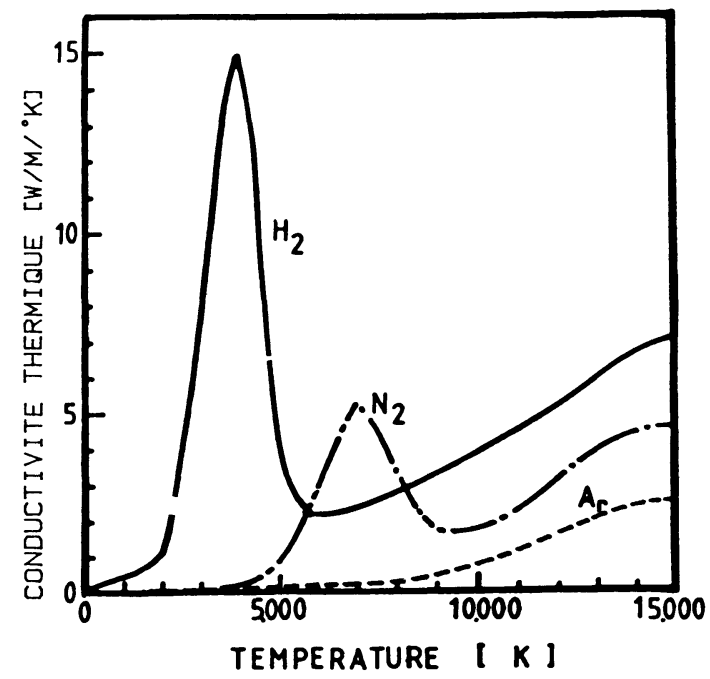

Fig. 3. - Evolution en fonction de la température et à la pression atmosphérique de la conductivité thermique de l'hydrogène, de l'azote et de l'argon $[35,36]$.

[Evolution with temperature, at atmospheric pressure, of the thermal conductivity of hydrogen, nitrogen and argon plasmas at equilibrium $[35,36]$.

avec la température des électrons $T_{\mathrm{e}}$ de la densité d'atomes et d'ions argon (à la pression atmosphérique). Les courbes de densité d'ions argon (égale à celle des électrons) se coupent toutes à environ $14000 \mathrm{~K}$ comme cela avait déjà été constaté [42]. Naturellement, pour une température électronique donnée la densité d'atome d'argon est d'autant plus élevée que $\theta$ est grand $\left(n_{\mathrm{Ar}}(\theta) \simeq n_{\mathrm{Ar}}(1) \times 1 / \theta\right)$ pour $T_{\mathrm{e}}<10000 \mathrm{~K}$, ce qui provient du fait que les densités électroniques et l'ionisation restent faibles pour ces températures (avec $\theta=10, T_{\mathrm{h}}=1000 \mathrm{~K}$ si $\left.T_{\mathrm{e}}=10000 \mathrm{~K}\right)$. 


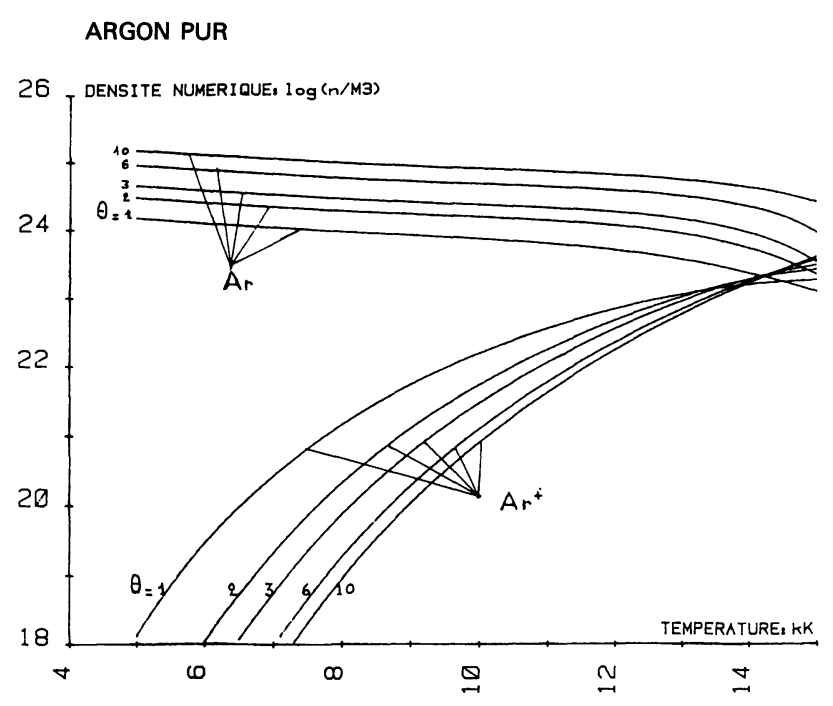

Fig. 4. - Evolution avec la température des électrons $T_{\mathrm{e}}$ et en fonction du rapport $\theta=T_{\mathrm{e}} / T_{\mathrm{h}}\left(T_{\mathrm{h}}\right.$ étant la température des particules lourdes) de la densité d'atomes et d'ions à la pression atmosphérique [43].

[Evolution with the electron temperature $T_{\mathrm{e}}$ and for various ratio $\theta=T_{\mathrm{e}} / T_{\mathrm{h}}\left(T_{\mathrm{h}}\right.$ being the temperature of the heavy particles) of the density of argon atoms and ions at atmospheric pressure [43].]

1.2.2.2 Propriétés de transport. - Le calcul de ces propriétés hors équilibre s'appuie, comme à l'équilibre, sur la résolution de l'équation intégro-différentielle de Boltzmann par la méthode d'Enskog [20].

En effet, à partir des relations établies à l'équilibre, Devoto [46] a montré que les électrons, d'une part, et les particules lourdes d'autre part sont en quasiéquilibre local à des températures différentes et que l'on peut donc chercher une solution de l'équation de Boltzmann pour les électrons d'une part et pour les particules lourdes d'autre part. Toutefois, comme l'a récemment montré Bonnefoi [43], en reprenant le calcul mathématique complet de la résolution de l'équation de Boltzmann à deux températures, les forces de diffusion utilisées par Devoto dans son modèle et établies dans les conditions d'équilibre ne peuvent être simplement généralisées au cas des deux températures et il convient d'en définir de nouvelles dominées par les gradients de pression et non plus de concentration. Cette modification, a priori non spectaculaire pour certains coefficients, introduit par contre une différence sensible au niveau de la diffusion et de la conductivité thermique réactionnelle $\lambda_{R}$, levant ainsi certains problèmes rencontrés par Hsu et al. [42] utilisant les équations de Devoto pour calculer un plasma d'argon à deux températures et obtenant un $\lambda_{R}$ augmentant considérablement lors du pic d'ionization. Il faut souligner que l'application du modèle à deux températures est relativement aisée puisqu'elle débouche sur des calculs numériques où interviennent exclusivement les intégrales de collision classiques, ce qui permet d'utili- ser ainsi des données testées (à l'équilibre) par un long usage.

A titre d'exemple, nous avons représenté sur la figure 5 tirée de [43] l'évolution, pour différentes valeurs de $\theta$, avec la température $T_{\mathrm{e}}$ de la conductivité thermique d'un mélange équimolaire hydrogène-argon. Comme on peut le constater pour $\theta=1$ le pic de dissociation de l'hydrogène qui se produit vers $3700 \mathrm{~K}$ (cf. Fig. 3) n'apparaît pas puisque les courbes commencent à $5000 \mathrm{~K}$. Par contre apparaît le pic de ionisation de l'hydrogène et de l'argon vers $14000 \mathrm{~K}$ (pics pratiquement confondus puisque les énergies d'ionisation sont similaires pour ces deux gaz). Pour $\theta=2$ le pic de dissociation de l'hydrogène se produit bien vers $T_{\mathrm{h}}=3700 \mathrm{~K}$ (soit $T_{\mathrm{e}}=6400 \mathrm{~K}$ ) car se sont effectivement les collisions entre particules lourdes qui sont responsables de la dissociation (pour $\theta=3$ ce pic se situe à $T_{\mathrm{e}} \simeq 11000 \mathrm{~K}$ ). L'énergie d'ionisation (fonction de $T_{\mathrm{e}}$ se produit toujours vers $14-15000 \mathrm{~K}$ et le pic d'ionisation est d'autant plus élevé que $\theta$ est grand (pour $\theta>3$ ) ce qui peut s'expliquer par la brutalité de l'ionisation qui se produit alors. Comme pour l'équilibre c'est encore bien la conductivité thermique réactionnelle qui domine largement.

\section{La génération des plasmas thermiques.}

2.1 REMARQUES GÉNÉRALES SUR LES ARCS. - Les générateurs à plasma d'arc ou « réchauffeur à arc», selon la dénomination de Pfender [47] sont maintenant largement répandus. Les différents gaz mis en contact avec l'arc atteignent des enthalpies de $10^{8} \mathrm{~kJ} / \mathrm{kg}$ et les quantités de gaz traitées peuvent aller jusqu'au $\mathrm{kg} / \mathrm{s}$.

HYDROGĖNE $50 \%$ - ARGON $50 \%$

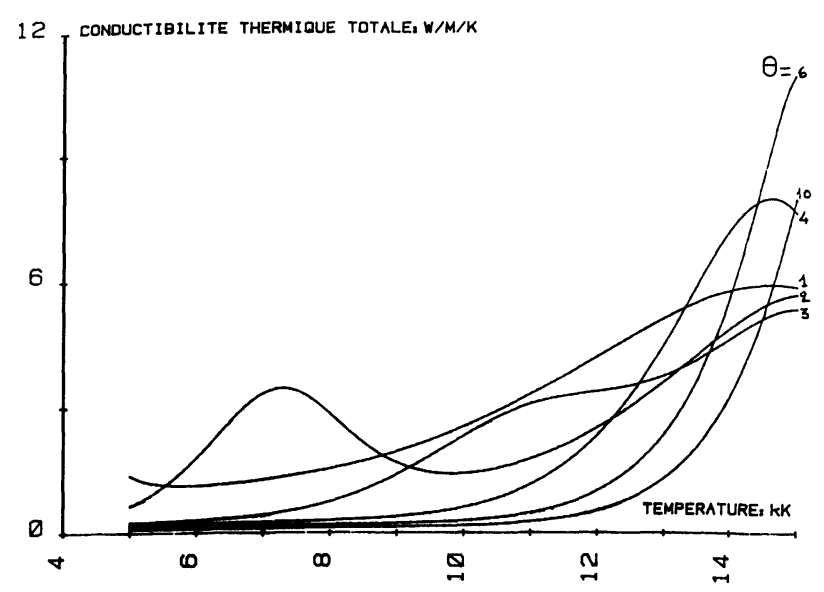

Fig. 5. - Evolution avec la température des électrons $T_{\mathrm{e}}$ et en fonction du rapport $\theta=T_{\mathrm{e}} / T_{\mathrm{h}}\left(T_{\mathrm{h}}\right.$ étant la température des particules lourdes) de la conductivité thermique d'un mélange $\mathrm{Ar}-\mathrm{H}_{2}(50 \%$ en moles) à la pression atmosphérique [43].

[Evolution with the electron temperature $T_{\mathrm{e}}$ and, for various ratio $\theta=T_{\mathrm{e}} / T_{\mathrm{h}}\left(T_{\mathrm{h}}\right.$ being the temperature of the heavy particles), of the thermal conductivity of a mixture $\mathrm{Ar}-\mathrm{H}_{2}$ (50\% in moles) at atmospheric pressure [43].] 
Nous renvoyons donc le lecteur aux nombreux articles de revues publiées sur le sujet et décrivant soit les générateurs à plasma [47 à 59], soit les fours à plasmas [60 à 69] et nous allons nous contenter de dégager brièvement les grandes lignes du fonctionnement de ces générateurs et fours à plasmas.

Dans tout ce qui suit nous allons présenter exclusivement les arcs soufflés, c'est-à-dire avec un jet de gaz en écoulement (utilisés dans les générateurs et les fours), gaz plasmagène dont la nature, le débit, la forme d'écoulement vont déterminer les caractéristiques de l'arc.

\section{Dans ces arcs :}

- la production des porteurs de charge dans la colonne d'arc est essentiellement due a l'ionisation thermique et non à l'ionisation de champ, ce dernier étant trop faible,

- les densités de courant atteignent des valeurs de $100 \mathrm{~A} / \mathrm{mm}^{2}$ et les densités d'énergie sont très grandes,

- les épaisseurs des zones de transition entre la colonne d'arc et les électrodes sont faibles : $10^{-3}$ à $10^{-4} \mathrm{~cm}$ pour la zone cathodique par exemple, d'où un mécanisme d'extraction très efficient avec des chutes cathodiques de l'ordre de $10 \mathrm{~V}$, entraînant des champs électriques de $10^{4}$ à $10^{5} \mathrm{~V} / \mathrm{cm}$,

- les densités de courant aux électrodes peuvent atteindre $10^{6} \mathrm{~A} / \mathrm{cm}^{2}$ avec des flux de $10^{6}$ à $10^{7} \mathrm{~W} / \mathrm{cm}^{2}$.

\subsubsection{La cathode. - Deux cas se présentent :}

a) Les cathodes chaudes. - L'attachement de l'arc est diffus avec l'émission thermo-ionique des électrons et des densités de courant de $10^{3}$ à $10^{4} \mathrm{~A} / \mathrm{cm}^{2}$ pour une cathode en tungstène par exemple. La forme de ces cathodes est essentiellement fonction de l'intensité de l'arc : cathodes en forme de tiges jusqu'à $1000 \mathrm{~A}$ environ; cathodes en forme de bouton noyées dans un support de cuivre énergiquement refroidi jusqu'à $5000 \mathrm{~A}$. En aucun cas ces cathodes thermoémissives ne peuvent devenir anode. La majorité de ces cathodes est en tungstène thorié avec, compte tenu des sévères problèmes d'érosion [70], une durée de vie de 100 à 200 heures pour des gaz non oxydants et à des pressions au maximum d'une dizaine d'atmosphères. Pour les gaz oxydants, on utilise le zirconium avec des cathodes de type bouton, mais la durée de vie est au mieux d'une dizaine d'heures, l'émission thermo-ionique étant due à la couche d'oxyde ou d'oxynitrure formée à la surface, couche isolante à froid nécessitant un accrochage sur le cuivre pour réinitier l'arc.

b) Les cathodes froides. - L'attachement de l'arc se fait sous la forme de un ou plusieurs points, se déplaçant rapidement et dont la surface est très petite (densité de courant entre $10^{-6}-10^{-8} \mathrm{~A} / \mathrm{cm}^{2}$ ). L'émission électronique est alors une émission de champ; c'est le cas des cathodes froides en cuivre, par exemple. Les effets secondaires dus aux vapeurs ou aux jets de plasma métalliques émis par la cathode peuvent exercer une forte influence sur les phénomènes au voisinage de la cathode ou parfois même sur l'arc dans son ensemble. La forme la plus fréquente est le tube. Du fait des densités de courant élevées à la cathode et des flux thermiques correspondants $\left(>10^{6} \mathrm{~W} / \mathrm{cm}^{2}\right)$, le refroidissement par circulation d'eau est insuffisant et le pied de l'arc doit être constamment déplacé avec un écoulement adapté et par un champ magnétique externe. Ces cathodes sont le plus souvent réalisées en cuivre et elles sont peu sensibles aux gaz agressifs. Elles n'ont pas de limitation de pression et leur durée de vie, suivant les gaz, varie de 150 à 600 h [71 à 74]. Contrairement aux cathodes chaudes, les cathodes froides peuvent devenir anode sans problème et donc fonctionner en courant alternatif. Toutefois, SKF [72] a montré que la durée de vie des électrodes cuivres était pratiquement doublées en courant continu par rapport au courant alternatif.

2.1.2 L'anode. - L'anode peut dissiper de 15 à $80 \%$ de la puissance de l'arc suivant la nature du gaz et surtout son débit, ses dimensions et sa forme géométrique, l'intensité de l'arc... Ceci implique donc une excellente protection thermique, soit par circulation d'eau (anode en cuivre correspondant pratiquement au cas général), soit par transpiration de gaz (anode poreuse en graphite). Les flux thermiques spécifiques à l'anode peuvent en effet atteindre $10^{5}$ à $10^{6} \mathrm{~W} / \mathrm{cm}^{2}$. La forme de l'anode peut être un simple tube, une tuyère ou un disque (dans ce dernier cas, l'anode est placée à l'extérieur du générateur). Afin de réduire l'érosion, on peut déplacer la zone d'attachement de l'arc par injection tangentielle de gaz et/ou par champ magnétique externe. Les durées de vie moyennes varient entre 150 à 500 heures.

Les flux de chaleur à l'anode ont été très étudiés [75 à 81]. En première approximation, l'expérience montre qu'ils sont linéairement proportionnels à l'intensité, mais que, pour une même intensité, ils varient seulement avec une puissance 0,2 ou 0,3 de la tension. Il convient donc, pour une même puissance, de travailler de préférence avec un voltage élevé, c'est-à-dire avec un arc long. Cependant l'augmentation de la longueur de l'arc est fonction de son confinement (paroi refroidie et éventuellement champ magnétique) et de son gainage gazeux. La longueur maximale que l'on peut atteindre sans pertes prohibitives dépend en fait $[50,52,57]$ de l'interaction entre les couches limites hydrodynamiques et thermiques qui s'établissent autour de l'arc et au voisinage des parois, les turbulences au-delà de cette zone de mélange augmentant très notablement les pertes aux parois.

2.1.3 La zone de constriction [47, 48]. - Dans les générateurs à basse ou moyenne enthalpie utilisés en chimie des plasmas, la stabilisation et la constriction de l'arc sont assurées soit par les parois refroidies généralement métalliques, soit par un vortex. 
La stabilisation par paroi consiste à enfermer l'arc dans un tube étroit intensivement refroidi et à un potentiel flottant. Toute excursion accidentelle de la colonne d'arc vers les parois est alors compensée par une augmentation de la conduction thermique vers la paroi entraînant une réduction de température de l'arc à cet endroit et donc une diminution de sa conductivité électrique qui le force à revenir à sa position d'équilibre. Si l'on veut augmenter la longueur de l'arc, une segmentation du tube de confinement est alors nécessaire afin d'éviter l'accrochage de l'arc entre la cathode et le tube, d'une' part, puis du tube et l'anode d'autre part, et certaines conditions de dimension (longueur, diamètre) ainsi que de débits de gaz doivent alors être respectées. Naturellement, le diamètre du tube de stabilisation doit, dans tous les cas, être inférieur au diamètre de l'arc libre si l'on veut que le phénomène de confinement intervienne. Les flux maximals admissibles aux parois étant de l'ordre de $10^{5} \mathrm{~kW} / \mathrm{m}^{2}$ environ, les tubes de stabilisation doivent être énergiquement refroidis, même par transpiration ou injection multiple de gaz.

Dans la stabilisation par écoulement tourbillonnaire, l'arc est confiné au centre d'un tube par un vortex intense de gaz ou de liquide, les forces centrifuges conduisant le fluide froid vers les parois de la tuyère-anode alors bien protégée thermiquement et confinant le plasma au centre de la tuyère.

\section{2 LES GÉNÉRATEURS.}

2.2.1 Générateurs à cathode chaude. - Dans la majorité des cas la cathode est un tungstène thorié. Ces générateurs ne peuvent donc fonctionner ni en courant alternatif, ni au-delà de $10 \mathrm{~atm}$, ni avec des gaz oxydants.

a) Cathode tige. - Ils sont essentiellement utilisés pour la projection avec des puissances de moins de $100 \mathrm{~kW}$ et ils correspondent à la première configuration proposée par Gage en 1957. Par exemple la figure 6 représente le générateur utilisé en projection au Laboratoire [82] où l'on voit que l'arc éclate entre la cathode en tungstène thorié $(3 \%$ de thorium pour faciliter l'émission thermo-ionique) et une tuyère anode en cuivre électrolytique (tuyère de forme très simple : convergent conique, cylindre dont la longueur et le diamètre doivent être adaptés au gaz utilisé [83]) refroidie par une circulation d'eau fortement laminée.

b) Cathode bouton. - Pour les puissances supérieures à $100 \mathrm{~kW}$, seules les cathodes de type bouton, noyées dans un support en cuivre fortement refroidi, sont utilisées. Le centrage sur la cathode est obtenu par l'injection des gaz en vortex comme pour le générateur russe $[84,85]$ représenté sur la figure 7 . Les cathodes peuvent être protégées par une injection annulaire d'argon en leur voisinage, ce qui autorise l'utilisation de gaz oxydant pour le vortex. Leur durée de vie (de l'ordre de $200 \mathrm{~h}$ ) est alors très supérieure à celle des cathodes en zirconium, utilisables

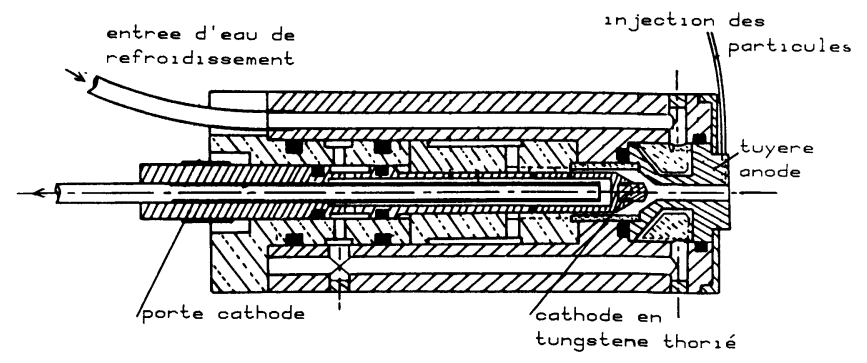

Fig. 6. - Générateur à plasma à cathode tige en tungstène thorié avec une tuyère anode en cuivre rouge refroidie par circulation d'eau utilisé entre 20 et $40 \mathrm{~kW}$ pour la projection au laboratoire [82].

[Plasma torch with a stick type cathode in thoriated tungsten with a water cooled nozzle anode in OFHC copper, used for spraying at the Laboratory $(20<P<40 \mathrm{~kW})[82]$.]

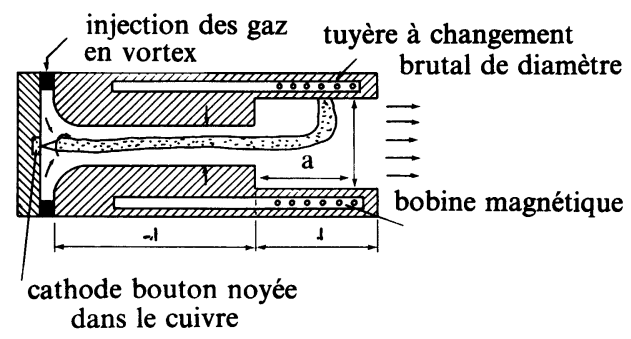

Fig. 7. - Générateur à cathode type bouton en tungstène ou zirconium avec injection des gaz en vortex et changement brutal de diamètre de la tuyère [84].

[Plasma torch with a button type cathode eitheir in tungsten or zirconium with a vortex gas injection and a steep variation of the nozzle diameter [84].]

avec les gaz oxydants, mais dont la durée de vie est inférieure à $20 \mathrm{~h}$.

Afin d'allonger l'arc et donc d'augmenter la tension à intensité égale (meilleur rendement thermique), deux solutions peuvent être utilisées :

- Une tuyère avec une variation brutale de diamètre créant, lorsque le rapport des deux diamètres est compris entre 1,5 et 2 , une zone de dépression (cf. a Fig. 7) tout de suite après le décrochement et où l'arc vient s'accrocher. De tels générateurs sont utilisés jusqu'à $1 \mathrm{MW}$ en U.R.S.S. [84].

- Une tuyère avec des injections multiples de gaz comme indiqué figure 8 . De tels générateurs sont utilisés jusqu'à $10 \mathrm{MW}[85,52,86]$. Des formules semi-empiriques permettent d'ailleurs de calculer les dimensions de ces générateurs en fonction du débit et de la nature du gaz, de la puissance et du courant électrique dissipés... [84, 85, 50 à 52].

c) Générateurs à stabilisation vortex liquide. Des générateurs fonctionnant avec de l'eau ou de l'alcool ont également été développés [87 à 89], comme, par exemple, celui d'Alu Suisse [89] de 


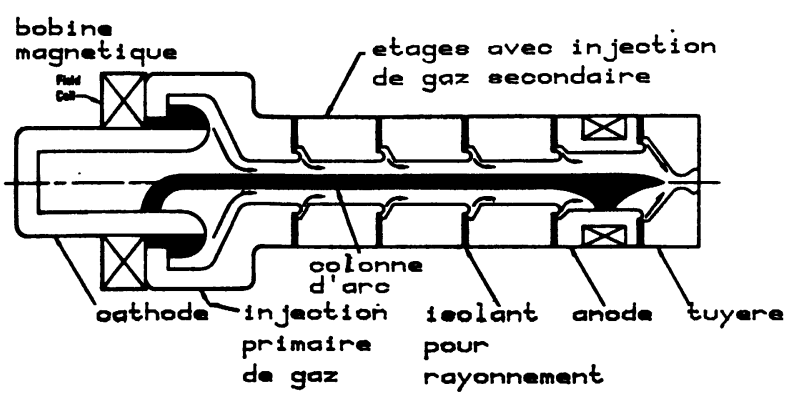

Fig. 8. - Schéma de principe du générateur d'Accurex avec sa tuyère à injection multiple de gaz afin d'allonger l'arc jusqu'à l'anode disposée à la sortie du générateur [86].

[Scheme of the Accurex plasma generator with its segmented nozzle allowing cold gas injection between the stages and thus increasing the length of the arc up to the anode disposed at the generator exit [86].]

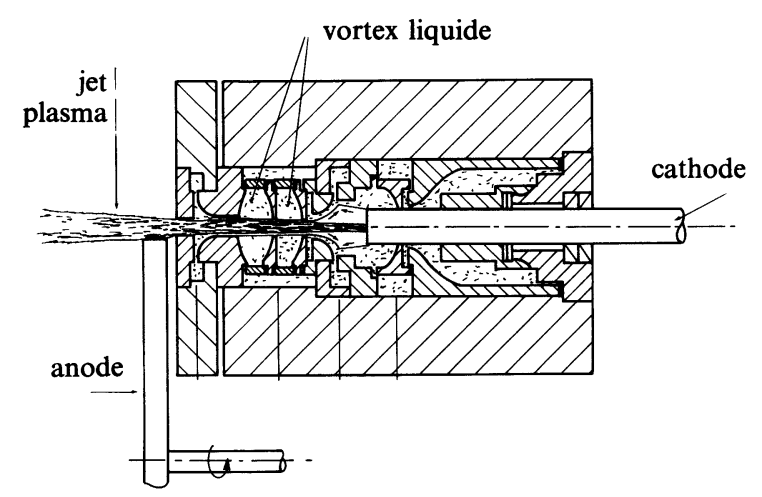

Fig. 9. - Générateur d'Alu Suisşe à vortex liquide avec une cathode en carbone consommable et une anode disque en cuivre refroidie et vaporisation du liquide au centre du vortex [89].

[Alu Suisse plasma torch with a liquid vortex, a consumable carbon cathode and an external copper rotating disk as anode. The plasma gas comes from the liquid vaporization in the vortex centre [89].]

$400 \mathrm{~kW}$ (Fig. 9). Les parois, comme nous l'avons indiqué, sont protégées par le vortex d'eau. L'anode est un disque de cuivre refroidi extérieur au générateur et la cathode en graphite consommable est avancée en continu.

\subsubsection{Les générateurs à cathodes froides.}

a) Courant continu. - Comme nous l'avons déjà dit, les électrodes tubulaires sont en cuivre et, outre leur refroidissement intensif, le pied d'arc doit être constamment déplacé par un champ magnétique et un vortex. Les puissances vont jusqu'à $2 \mathrm{MW}$ pour des débits de gaz, oxydants ou non, qui varient du $\mathrm{g} / \mathrm{s}$ au kg/s [85, 90, 91].

Signalons que ce type de générateur avec des électrodes d'acier et des puissances de $8 \mathrm{MW}$ est utilisé depuis la guerre de 40 pour la production de l'acétylène à partir des hydrocarbures par Hüls en Allemagne [92, 93]. b) Courant alternatif. - Le problème avec le courant alternatif est d'éviter l'extinction de l'arc lors du changement d'alternance. Ceci peut être fait $[94,95]$ en superposant un courant haute fréquence au courant d'arc. Cependant, il faut alors avoir des puissances HF qui atteignent 10 à $15 \%$ de la source alternative, ce qui grève notablement le prix de l'alimentation. L'autre solution consiste à faire en sorte que la distance entre les électrodes soit suffisamment petite pour qu'avec la tension d'alimentation en circuit ouvert le claquage se produise. C'est, par exemple, la solution de Westinghouse [74] dont le générateur (jusqu'à $2 \mathrm{MW}$ ) est présenté sur la figure 10. Il est intéressant de remarquer que la société S.K.F., en utilisant un générateur dérivé de celui de Westinghouse avec du courant redressé, a multiplié par 2 la durée de vie des électrodes. C'est vers la solution du continu que s'orientent également Jeumont, Schneider et SNIAS qui développent ce type de torche en France.

Signalons également des générateurs en courant triphasé où les trois électrodes sont gainées par le gaz plasmagène et où l'allumage est obtenu avec une petite torche à plasma à courant continu qui ionise le gaz entre les électrodes [96 à 98].

2.2.3 Les problèmes posés par l'utilisation de ces générateurs. - Ces problèmes sont liés aux caractéristiques des générateurs :

a) Temps de séjour. - Les températures et les vitesses des plasmas produits varient, sur l'axe en sortie de tuyères, entre 6000 et $12000 \mathrm{~K}$ et entre 30 et $1000 \mathrm{~m} / \mathrm{s}$. On conçoit donc que les produits à traiter y soient entraînés rapidement (cf. §4) et que les temps de séjour, dans des plasmas de quelques dizaines de centimètres au plus, y soient de l'ordre de grandeur de la ms, c'est-à-dire suffisants pour des réactions

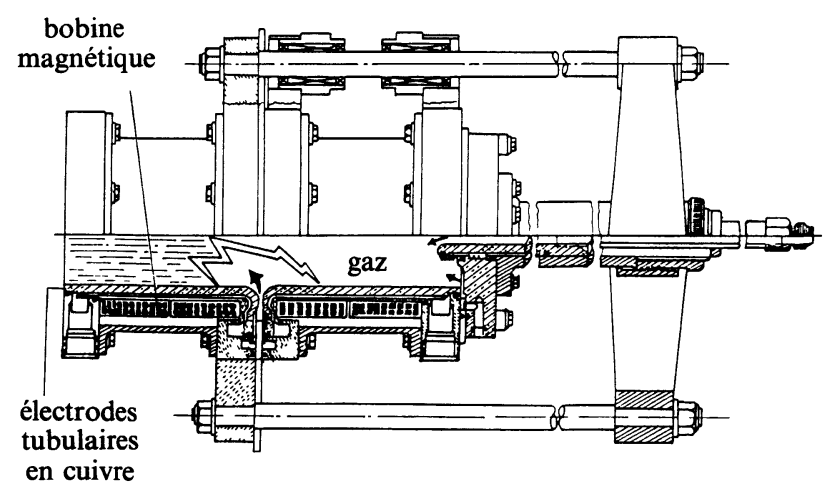

Fig. 10. - Générateur de Westinghouse à électrode tubulaire en cuivre et rotation de l'arc par injection centrale tourbillonnaire et champ magnétique (puissance 0,5 à 3,5 MW) [34, 74].

[Westinghouse plasma torch with tubular coaxial copper electrodes, the arc rotation being due to the gas injection in vortex between the two electrodes and a magnetic coil $[34,74]$.] 
chimiques en phase gazeuse $\left(10^{-6} \mathrm{~s}\right.$ en moyenne pour une réaction élémentaire), pour une fusion des particules solides, voire une évaporation si leur diamètre ne dépasse pas $10 \mu \mathrm{m}$ (cf. $\S 4$ ), mais totalement insuffisants pour leur transformation chimique complète en présence de réactifs.

b) Inhomogénéité du traitement. - Les gradients radiaux peuvent être élevés (jusqu'à $4000 \mathrm{~K} / \mathrm{mm}$ et $200 \mathrm{~m} / \mathrm{s} / \mathrm{mm}$ ), ce qui peut rendre les traitements des produits très hétérogènes suivant leurs trajectoires.

c) Réactivité du plasma. - Les vitesses de chauffage des gaz peuvent atteindre $10^{9} \mathrm{~K} / \mathrm{s}$ et dans beaucoup de cas les gaz se trouvent dans des états excités hors d'équilibre (notamment vibrationnels) pouvant favoriser certaines réactions et permettre d'obtenir des taux de conversion supérieurs à ceux de l'équilibre $[99,100]$.

d) Introduction des produits gazeux. - Le problème majeur reste cependant l'introduction des réactifs dans le plasma. En effet, celui-ci reste très visqueux (viscosité entre un et deux ordres de grandeur supérieure à celle du gaz froid), sans compter que cette viscosité est augmentée par les turbulence [101] :

- Pour le gaz plasma lui-même, on constate que seulement 30 à $40 \%$ des gaz passent dans la zone centrale chaude (peut être $60 \%$ pour les générateurs avec vortex), le reste circulant en périphérie à des températures inférieures à $2000 \mathrm{~K}$ en moyenne.

- Si l'on veut injecter un gaz froid dans le plasma en écoulement (au-delà de l'arc électrique pour éviter tout problème avec les électrodes sauf lorsqu'elles sont en cuivre pour certains gaz), on constate $[102,103]$ que les gaz froids ne pénètrent pas dans le plasma, entraînant un phénomène de striction du gaz plasma et une sorte de rebondissement du gaz froid qui ne réussit à se mélanger que bien au-delà des zones chaudes, même si on lui donne une forte énergie cinétique [102, 103].

e) Introduction des produits solides à traiter. Pour pénétrer dans le plasma, en principe, il suffit de donner aux particules une énergie cinétique suffisante pour pénétrer dans l'écoulement visqueux. Ceci cependant pose déjà un problème si l'on traite, comme c'est le cas général, des particules de granulométrie assez large. En effet, si l'on adapte la vitesse du gaz porteur aux particules du centre de la distribution, les plus fines risquent de rebondir sur le jet et les plus grosses de le traverser.

On a aussi mis en évidence récemment [104] un autre phénomène qui s'oppose à la pénétration des particules : les forces de thermophorèses dirigées en sens contraire de la vitesse d'injection et proportionnéés à $\mathrm{d} T / \mathrm{d} r$. Ces forces sont donc d'autant plus grandes que les gradients de température sont élevés et il faut donc diminuer ces derniers autant que faire se peut en augmentant, par exemple, le diamètre des tuyères et/ou en utilisant des tuyères à changement de diamètre.

Tous ces problèmes ont donc entrainé le développement des fours à plasma, mieux adaptés au traitement des phases solides que les générateurs.

2. 3 Les fours À PLASMa. - En moyenne les temps de séjour des particules solides dans les générateurs décrits au paragraphe précédent varient de 1 à $10 \mathrm{~ms}$ ce qui est tout à fait insuffisant pour transférer l'énergie du plasma au solide et réaliser une réaction chimique. Les fours ont donc été conçus pour améliorer le temps de séjour $t_{\mathrm{s}}$ et beaucoup de technologies ont été proposées au cours des quinze dernières années [60 à 69].

\subsubsection{Amélioration du temps de séjour.}

a) Fours à contre courant $\left(t_{\mathrm{s}} \sim 10^{-2}\right.$ à $\left.10^{-1} \mathrm{~s}\right)$. C'est par exemple, le cas du four à parois chaudes de Segworth pour la métallurgie extractive du fer et des ferrochromes [105] alimenté en courant triphasé avec trois électrodes creuses en carbone, donc consommables (Fig. 11). Les réactifs solides tombent par gravité le long de la colonne d'évacuation du plasma, traversent l'arc et finissent par être traités dans le bain des produits en fusion où il convient cependant d'assurer une homogénéisation, par exemple, avec un chauffage HF annexe et un brassage magnétique.

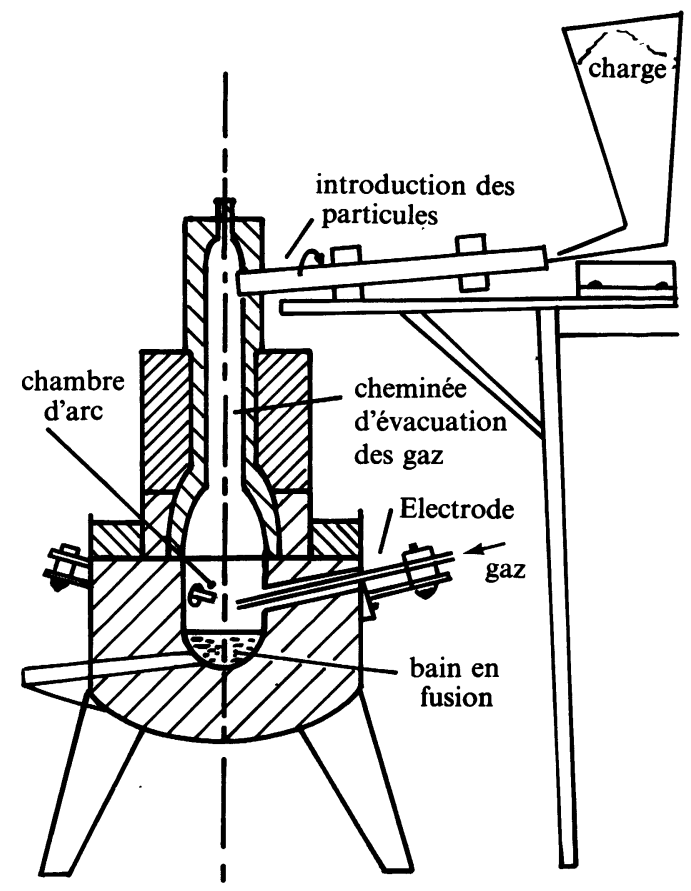

Fig. 11. - Four à plasma à contre-courant avec trois électrodes creuses en carbone développé à l'Université de Toronto [105].

[Plasma furnace with three hollow carbon electrodes, the particles being introduced countercurrent to the hot gas exhaust flow (developed at the University of Toronto) [105].] 
b) Fours à plasma expansé $\left(t_{\mathrm{s}}: 10^{-2}\right.$ à $\left.10^{-1} \mathrm{~s}\right)$. On tente d'améliorer le contact plasma-particules en forçant le plasma à occuper la plus grande partie du volume du réacteur par épanouissement, soit :

- par rotation de la paroi comme dans le réacteur de $30 \mathrm{~kW}$ développé par Wymann [106] et utilisé pour l'évaporation de réfractaires comme l'alumine;

- par rotation de la cathode comme le four de Tetronics [107] de $200 \mathrm{~kW}$ où la cathode précesse avec une anode en forme d'anneau formant un tronc de cône de plasma dans lequel les particules, injectées tangentiellement au voisinage du sommet du tronc du cône, décriraient, du fait de la viscosité du plasma, une hélice dans le cône du plasma. Les produits obtenus sont alors recueillis au fond du creuset où ils sont encore traités par le plasma (convection, rayonnement);

- par champ magnétique comme dans le procédé AVCO $[108,109]$ pour la synthèse de l'acétylène à partir du charbon;

- par champ magnétique avec des arcs sous forte tension pulsée (jusqu'à $45 \mathrm{~kW}$ ) ou sous basse tension avec des rotors auto-commutants [110].

c) Fours à arc long. - Pour faciliter la pénétration des particules et obtenir un meilleur temps de séjour on utilise l'effet Maecker [111 à 115], effet magnéto-hydrodynamique qui crée une dépression en pointe de cathode [111], permettant d'entraîner les particules dans l'écoulement plasma très visqueux. De plus en allongeant l'arc et en l'étendant pour diminuer sa vitesse, le temps de séjour augmente. C'est par exemple, les fours de sphéroïdisation réalisés par IONARC, Sheer ou Sayce où l'injection se fait autour de la cathode et où l'arc se referme, soit sur trois électrodes en carbone consommables (puissance $300 \mathrm{~kW}$ ) [112] (Fig. 12), soit sur trois anodes poreuses alimentées en gaz [113,114], soit sur trois jets de plasma produits par des générateurs à courant continu [115].

Il faut souligner que dans ce type de four l'allongement de l'arc est fonction à la fois de la vitesse de l'écoulement autour de la cathode [116] et de celle des gaz d'écran que l'on injecte autour de celle-ci (gaz d'écran qui est parfois réactif, $\mathrm{CO}_{2}$ par exemple, et qu'il convient donc de ne pas laisser entrer en contact avec la cathode).

2.3.2 Fours à charge liquide. - La charge en fusion est maintenue au contact du plasma pendant des temps supérieurs à la seconde permettant la diffusion des réactifs gazeux dans la phase condensée et donc la réaction chimique.

a) Fours à parois tournantes

- Soit à axe horizontal comme celui du CEA [117] où le matériau en auto-creuset est centrifugé à l'intérieur d'une enceinte en rotation, le plasma, généré par superposition entre deux générateurs disposés à l'extérieur du four, se développant le long de l'axe du

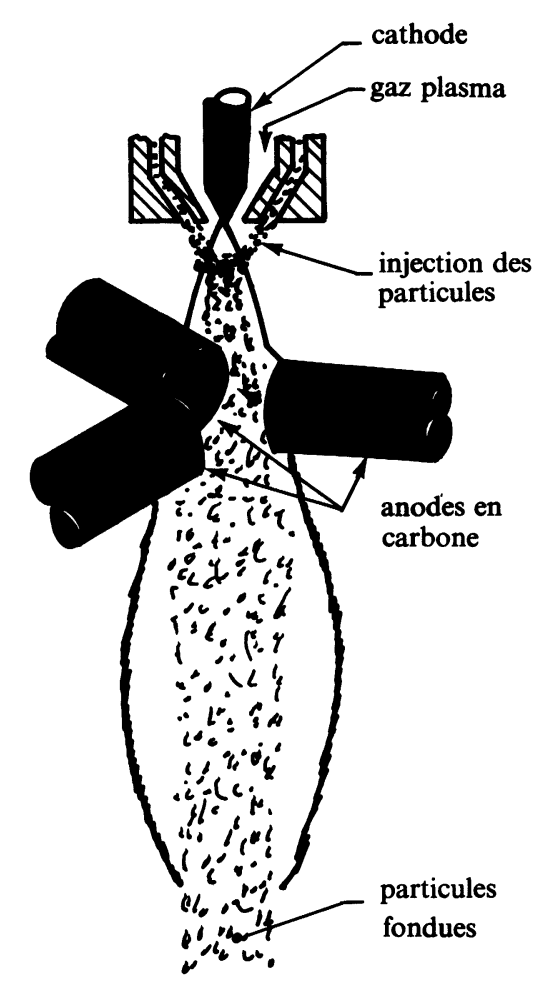

Fig. 12. - Four à effet Maecker d'Ionarc avec injection des particules au voisinage de la pointe de la cathode et fermeture de l'arc sur 3 anodes en carbone [112].

[Ionarc plasma furnace using Maecker's effect for the particles penetration near the cathode tip, the arc being struck to three consumable carbon anode [112].]

cylindre et le four étant renversé périodiquement pour permettre la coulée du matériau en fusion.

- Soit à axe vertical comme celui de Sayce [118] où les particules introduites dans un plasma généré par superposition (CC) entre deux générateurs à axes concurrents, sont centrifugées sur un paraboloïde à axe vertical. Si ce four peut être alimenté en continu sans problème (puissance de $60 \mathrm{~kW}$ ), l'énergie rayonnée vers la voûte par le paraboloïde nécessite un refroidissement énergique de celle-ci.

L'avantage de ces fours est que le confinement de la charge autour du plasma permet un très bon transfert d'énergie (jusqu'à $70 \%$ ).

b) Fours avec effet d'électrode sur la charge. Lorsque le matériau liquide sert d'anode, une grande partie de l'énergie du plasma perdue à l'anode (celle-ci peut atteindre $50 \%$ de l'énergie fournie au plasma [76 à 81]) est récupérée dans la charge, ce qui améliore d'autant le transfert thermique. 60 à $70 \%$ de l'énergie perdue à l'anode est due à la collection des électrons qui a lieu sur une petite surface de l'anode (surface de quelques $\mathrm{mm}^{2}$ ) qui se déplace assez rapidement. Le problème est d'obtenir la fusion de la charge qui, dans le cas des oxydes, devient conductrice et donc il convient de veiller particulièrement à 
l'introduction des particules dans le plasma. C'est le cas par exemple :

- du four de la Bethlehem Steel [119] où les particules injectées tangentiellement au-dessus de l'anode viennent s'accrocher sur celle-ci et former un film ruisselant (cf. Fig. 13).

Cependant, un des inconvénients de ce four est le problème de l'injection de la poudre [120] : un débit trop faible entraîne une vaporisation importante (plus de $25 \%$ du minerai), un débit trop fort diminue la conductivité électrique du film, de telle sorte que l'arc vient s'accrocher au-dessus du film ruisselant, diminuant ainsi considérablement le transfert thermique. Il faut cependant noter que Bethlehem a été un des premiers à démontrer l'effet de taille avec les réacteurs plasmas : si leur premier four de $100 \mathrm{~kW}$ consommait $5 \mathrm{kWh} / \mathrm{lb}$ de fer produit à partir d'un minerai, le four de $1 \mathrm{MW}$ ne consommait plus que $2 \mathrm{kWh} / \mathrm{lb}$ de fer produit à partir du même minerai. Soulignons également que les caractéristiques de fonctionnement de ces fours $(1000 \mathrm{~V}-1000 \mathrm{~A}$ pour $1 \mathrm{MW}$ ) sont loin des caractéristiques habituelles des fours à $\operatorname{arc}(100 \mathrm{~V}-10000 \mathrm{~A}$ pour un petit four).

- Le deuxième type de four vers lequel on semble maintenant s'orienter est celui du transfert de l'arc sur la charge : Noranda pour la production de molybdène à partir de la molybdénite [121] et depuis 1982 Davee McKee en Angleterre, à partir d'une licence

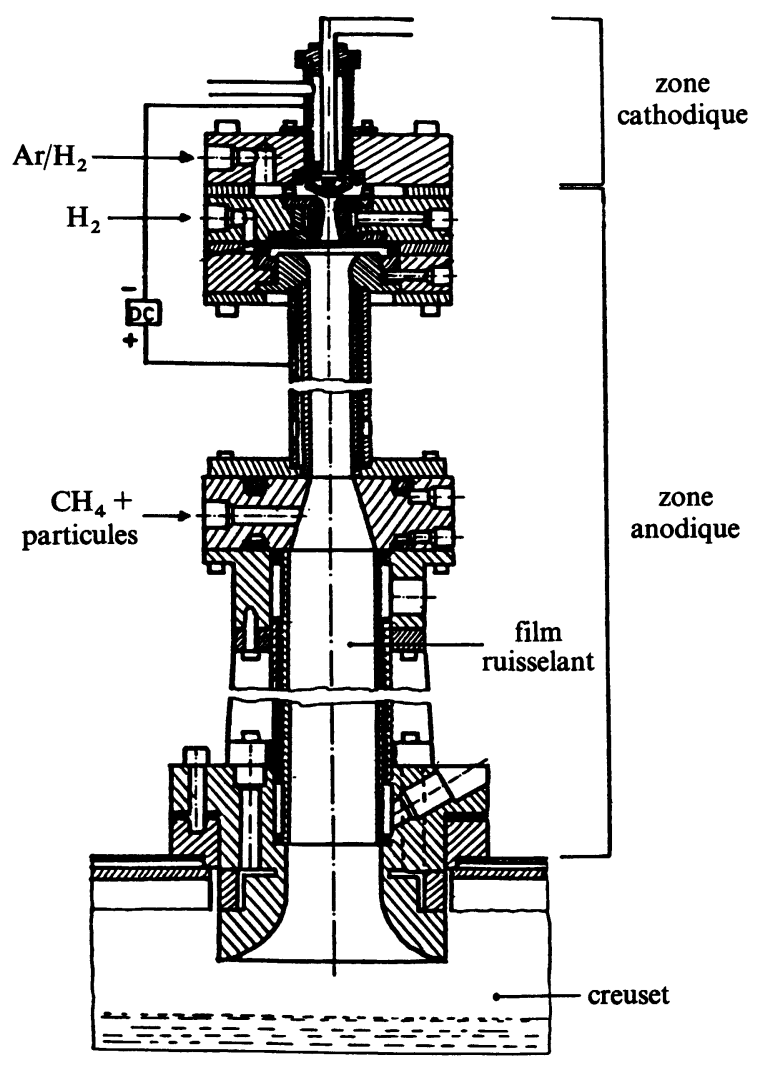

Fig. 13. - Four à film ruisselant de Bethlehem Steel [119].

[Falling film plasma furnace developed by Bethlehem Steel Co. [119].]
Noranda, pour la production de ferro-chrome, Foster Wheeler pour la refusion [122] avec la commercialisation d'un four de $8 \mathrm{MW}$ en Afrique du Sud chez Middelburg, la refusion en plasma à l'échelle de 19,8 MW chez VEP Edelstahlwerhe à Freital en R.D.A. [123 à 125], four commercialisé par Voest Alpine en Autriche, le laboratoire de Limoges pour la production de ferrochrome [120,66, 68], Mintech en Afrique du Sud pour les ferrochromes [126].

Dans tous ces fours l'arc éclate entre la pointe de la cathode, généralement en tungstène thorié ou en carbone, et le bain de fusion servant d'anode avec une électrode de fond de creuset en métal ou en carbone suivant la nature du matériau à traiter. Les particules, lorsque ces fours sont alimentés en particules (pratiquement tous sauf celui de Foster Wheeler ou des allemands de l'Est) sont introduites au voisinage de la pointe de cathode pour bénéficier de l'effet Maecker. Les arcs sont cependant très longs (de 20 à $80 \mathrm{~cm}$ ), leur longueur étant déterminée par la vitesse de l'écoulement des gaz injectés tout.autour de la cathode (et qui ne doit pas dépasser $50 \mathrm{~m} / \mathrm{s}$ pour éviter l'entraînement de la tache cathodique liquide) ou en périphérie de celle-ci en gainage. Une partie des particules (10 à $20 \%$ ) reste en périphérie de l'arc, captant une grande partie de son rayonnement qui peut atteindre facilement $50 \%$ de la puissance de l'arc [120], [116] et protégeant ainsi les parois du four. Afin de mieux répartir la puissance de l'arc sur la charge à traiter on utilise une cathode précessive autour de l'axe du four comme dans le montage de Foster Wheeler [122] (cf. Fig. 14) ou plusieurs torches comme dans le montage Est Allemand [123], de récentes études indiquant d'ailleurs la possibilité éventuelle de les alimenter avec une seule source [127], ce qui doit limiter ainsi largement les coûts de l'appareillage.

Signalons enfin des fours de refusion pour la purification de métaux à forte valeur ajoutée développés

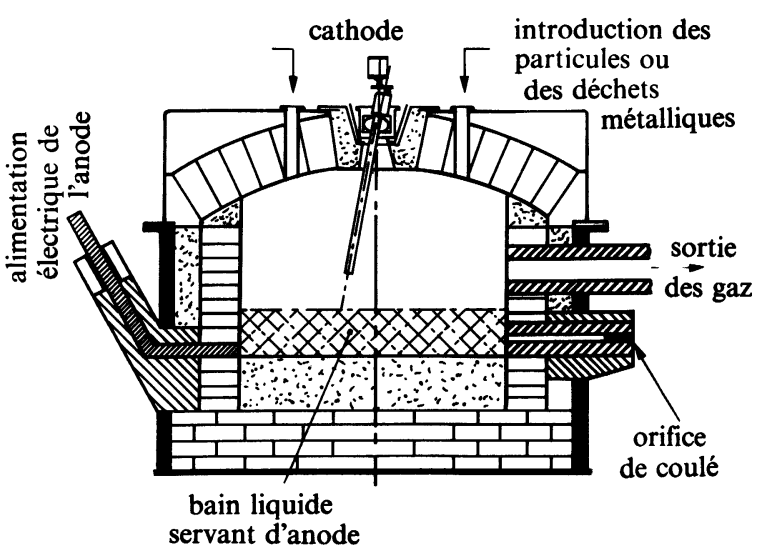

Fig. 14. - Four à cathode précessive de Foster Wheeler avec fermeture de l'arc sur le bain de fusion [122].

[Foster Wheeler's plasma furnace with a precessive cathode, the molten bath being used as anode [122].] 
en U.R.S.S., au Japon, en Europe et aux U.S.A. [128 à 132]. Dans ces fours, soit le métal à fondre sous forme de barreaux sert d'anode [129], soit les particules tombent dans un arc transféré entre une cathode gainée par écoulement de gaz et/ou un bain de fusion [131], ou l'extrémité d'un lingot refroidi et dont seule la surface est en fusion [132].

2.3.3 Fours à cavité de coke. - La société S.K.F. $[133,134,72]$, a réussi à concilier les avantages du plasma avec ceux des hauts-fourneaux en imaginant le dispositif suivant : en sortie d'un générateur du type Westinghouse, alimenté avec un mélange de gaz (mélange $\mathrm{CO}_{2}, \mathrm{CO}, \mathrm{H}_{2}$ ) porté à une température enthalpique moyenne de $4000 \mathrm{~K}$, avec une énergie de 4 à $8 \mathrm{kWh} / \mathrm{Nm}^{3}$, on injecte le minerai pulvérulent à traiter et da charbon. Ce générateur débouche dans un four rempli de coke où les gaz plasma créent rapidement une cavité (cf. Fig. 15) à une température de paroi de l'ordre de $2500 \mathrm{~K}$ [72] dans laquelle se produit la réaction endothermique de réduction entre l'oxyde et le réducteur $(85 \%$ charbon, $15 \%$ coke). Comme il n'y a pas d'admission d'air, toute la chaleur nécessaire provient du gaz plasma.

En présence d'un excès de coke, seuls $\mathrm{CO}$ et $\mathrm{H}_{2}$ sont formés et ces gaz s'élèvent dans le fourneau. Leur volume étant faible, les particules injectées en suspension sont piégées à la surface du coke et éven-

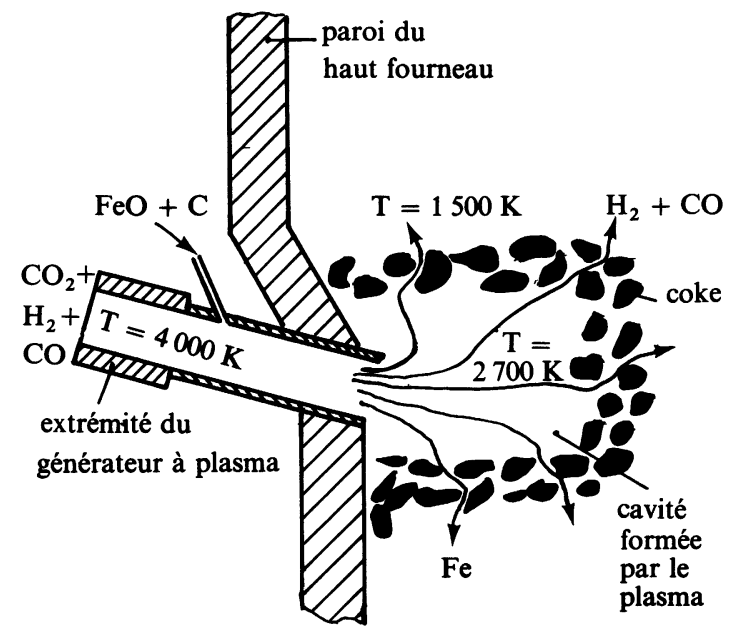

Fig. 15. - Schéma de principe du procédé Plasmamelt de S.K.F. avec un générateur à plasma alimenté en $\mathrm{CO}_{2}+\mathrm{H}_{2}+$ $\mathrm{CO}$ comme gaz plasmagène et où est injecté un mélange de fer préréduit et de carbone (charbon, huile lourde, ...) les gaz plasma forment une cavité dans le coke dont est rempli le haut fourneau, cavité où les particules à réduire viennent se piéger sur les parois [72].

[Scheme of the Plasmamelt process of S.K.F. using a plasma torch with a mixture of $\mathrm{CO}_{2}, \mathrm{H}_{2}$ and $\mathrm{CO}$ as plasma gas and where is injected a mixture of prereduced iron ore and carbon (coal, heavy oil, ...) particles. The plasma gas creates a cavity in the coke filling the furnace, cavity on the wall of which the particles to be reduced are trapped and thus have a sufficient long residence time to be reduced [72].] tuellement reviennent dans la zone de réaction qui s'étend sur une profondeur de $25 \mathrm{~cm}$ environ autour de la cavité. On peut même utiliser les gaz sortant du fourneau pour la préréduction du minerai avant son injection en sortie du générateur à plasma. Ce dispositif a été testé sur les minerais de fer et de chromite. Le temps de séjour des particules est très long et la réduction est donc complète, le métal liquide coule lentement vers le fond du fourneau. De plus, la sensibilité de la réaction au pouvoir réducteur des gaz plasmas est moins grande que dans les dispositifs à arc transféré dans la mesure où le coke joue un rôle tampon.

2.3.4 Conclusion, résumé. - Contrairement à la flamme, l'énergie fournie au plasma n'est pas limitée par la chaleur de combustion (on peut atteindre des enthalpies de $100000 \mathrm{~kJ} / \mathrm{kg}$ et la température du plasma peut atteindre facilement $10000 \mathrm{~K}$.

L'effet de transfert thermique est important (cf. $\S 3.3)$ : pour les gaz di ou polyatomiques, le coefficient de transfert présente une augmentation très brutale dès lors que la température du plasma dépasse la température de dissociation (il peut, par rapport à une flamme être multiplié par 20 avec un plasma d'hydrogène à $T>4500 \mathrm{~K}$ ).

Les réacteurs sont très compacts (on peut dissiper $1 \mathrm{MW}$ dans moins de $1 \mathrm{dm}^{3}$ ), ceci induit des mises en route et des arrêts très rapides ainsi que des cinétiques ultrarapides et souvent hors équilibre.

De plus en plus, les générateurs peuvent utiliser des fluides comme l'air, l'eau, le $\mathrm{CO}_{2}$, l'hydrogène et ils ne sont plus pénalisés par le coût de gaz comme l'argon ou l'hélium.

En contrepartie, il convient de contrôler soigneusement les phénomènes suivants :

- l'injection des réactifs afin d'être certain qu'ils pénètrent dans le plasma;

- les temps de séjour (diminution des vitesses d'écoulement) et la réduction des gradients radiaux de température et de vitesse (amélioration de l'homogénéité du traitement).

En conclusion, ces propriétés donnent au four à plasma les avantages suivants sur les fours électriques conventionnels :

- très grande vitesse de fusion

- contamination par le carbone négligeable ou nulle

- pas de consommation d'électrode

- produits ayant les qualités de ceux obtenus sous vide grâce au contrôle de l'atmosphère du four

- fonctionnement en continu

- coût d'installation plus faible que ceux des fours à vide à induction et/ou des fours électriques couplés à un dégazage sous vide

- réduction des pertes de métal à forte tension de vapeur

- refusion couplée à la déoxydation

- faible bruit, faibles variations de tension, moins de pertes de réfractaires. 
3. Modélisation et caractéristiques des écoulements plasma réactifs ou non avec ou sans particules.

3.1 L'ÉCOULEMENT NON RÉACTIF. - Dans l'état actuel des connaissances, pratiquement seuls les arcs à courant continu ont été modélisés à ce jour. En dépit d'un effort intensif de recherche au cours des trente dernières années, les phénomènes de base au voisinage des électrodes sont encore mal compris $[70,71$, 75 à 81]. La principale difficulté provient du fait qu'il est encore quasi impossible d'effectuer des mesures détaillées et précises dans ces̀ régions dont les épaisseurs sont de l'ordre de $10^{-3}$ à $10^{-2} \mathrm{~mm}$ et où les effets de non-équilibre sont considérables. Toutefois sans que les phénomènes élémentaires et nécessairement hors équilibre qui ont lieu dans la zone cathodique soient vraiment pris en compte, on commence [135] à estimer des distributions de courant depuis la pointe de la cathode, distributions dont dépendent fortement le comportement de l'arc. Des distributions de la forme

$$
J(r)=J_{\max } \exp \left[-c r^{0,5}\right]
$$

se sont avérées réalistes [136] où $J_{\max }$ est la densité de courant sur la pointe de la cathode, estimée expérimentalement à partir du diamètre de la tache cathodique, d'une cathode thermo-émissive, où $r$ est le rayon de l'arc et où $c$ est une constante calculée à partir du bilan de courant [137].

C'est pourquoi depuis 1975 le travail de modélisation a été essentiellement limité à la colonne d'arc ou au jet libre de plasma s'écoulant après la tuyèreanode.

\subsubsection{Calculs à l'équilibre.}

a) Les équations. - Les hypothèses de base sont les suivantes : équilibre thermodynamique local, l'arc ou le jet est en régime permanent et de symétrie axiale, les effets de thermodiffusion sont négligés, les effets de gravité et de dissipation visqueuse sont négligeables, les jets sont subsoniques, les écoulements sont laminaires, les propriétés thermodynamiques et de transport ne dépendent que de la température (gradients de pression négligeables).

On exprime alors les équations de conservation en coordonnées cylindriques $(z, r, \theta)$ [81] : conservation de la masse, de la quantité de mouvement (où l'on néglige le flux de courant radial/à celui axial), de l'énergie en tenant compte de la loi d'Ohm liant le courant de l'arc $I$ aux densités de courant et à la conductivité électrique du plasma.

C'est-à-dire par exemple [137] en négligeant les gradients de vitesse et de température axiaux par rapport aux radiaux, ainsi que le champ magnétique propre.

Conservation de la masse :

$$
\frac{\partial}{\partial z}(\rho u)+\frac{1}{r}(r \rho v)=0 .
$$

Conservation du moment axial :

$$
\rho u \frac{\partial u}{\partial z}+\rho v \frac{\partial u}{\partial r}=-\frac{\partial p}{\partial z}+\frac{1}{r} \frac{\partial}{\partial r}\left(r \mu \frac{\partial u}{\partial r}\right) .
$$

Conservation de l'énergie :

$$
\begin{aligned}
\rho u \frac{\partial h}{\partial z}+\rho v \frac{\partial h}{\partial r}=\frac{1}{r} \frac{\partial}{\partial r}\left(r \kappa \frac{\partial T}{\partial r}\right)+\sigma E_{z}^{2} & -P_{\mathrm{r}}+ \\
& +\frac{5}{2} \mathbf{j} \cdot \operatorname{grad} T .
\end{aligned}
$$

Dans ces équations $\rho$ est la densité massique du plasma, $u$ et $v$ les composantes axiales et radiales de la vitesse, $p$ la pression, $E_{z}$ le champ électrique axial, $j$ la densité du courant, $h, \mu, \kappa, \sigma, P_{\mathrm{r}}$ respectivement l'enthalpie, la viscosité, la conductivité thermique, la conductivité électrique et le rayonnement du plasma.

Les deux premières équations sont essentiellement celles de Navier Stokes (on a négligé les termes supplémentaires dus à la présence des particules chargées et du champ magnétique propre), le terme incluant la viscosité correspondant au tenseur des contraintes.

Dans l'équation d'énergie, le terme de gauche représente le transport convectif et les termes de droite respectivement les pertes conductives, l'énergie gagnée par les particules chargées dans le champ, les pertes radiatives et le flux enthalpique des électrons (si la colonne d'arc est complètement développée $\mathbf{j}$ et grad $T$ sont orthogonaux et ce terme disparaît).

A ces équations s'ajoute la loi d'Ohm

$$
E_{z}=\frac{I_{z}}{2 \Pi} \frac{1}{\int_{0}^{R} r . \sigma . \mathrm{d} r}
$$

où $R$ est le rayon de l'arc et $I_{z}$ le courant électrique.

Avec les conditions aux limites appropriées pour les variations du champ : $u, v, p, T, I$ et $\mathbf{j}$ ces équations peuvent être résolues si les propriétés thermodynamiques et de transport du plasma sont connues (dans l'hypothèse de l'ETL elles ne dépendent que de la température pour une pression donnée, supposée généralement constante dans ce type de calcul).

Les méthodes de calcul de différences finies développées par Patankar [138] ont considérablement amélioré les calculs qui peuvent facilement diverger $\mathrm{du}$ fait des erreurs numériques introduites par les variations brutales de densité devenant alors très sensibles au choix du pas de la grille [139].

Des calculs ont également été développés pour prendre en compte les phénomènes de turbulence. On écrit par exemple la viscosité comme la somme de deux termes : $\mu_{\text {eff }}=\mu_{\mathrm{t}}+\mu_{1}$ où $\mu_{\mathrm{t}}$ est la viscosité calculée dans le cadre du modèle de turbulence $K-\varepsilon$ exprimant la conservation de l'énergie cinétique de turbulence $K$ et celle du taux de dissipation $\varepsilon$ de l'énergie de turbulence où l'on résout les équations de conservation de la turbulence [140 à 142]. 
b) Résultats obtenus et comparaisons aux mesures. - Les premières études dans les années 60 furent celles de Stine et Watson [143] qui, supposant négligeable le champ magnétique self induit et que $\frac{\partial T}{\partial z}=v=0$, ont ainsi découplé les équations de quantité de mouvement et d'énergie ce qui conduit à la célèbre équation d'Ellenbaas Eller :

$$
\frac{1}{r} \frac{\partial}{\partial r}\left(r \kappa \frac{\partial T}{\partial r}\right)+\sigma E_{z}^{2}-P_{\mathrm{r}}=0 .
$$

En 1967 Watson et Pegot [144] établissaient les solutions complètes pour un arc à l'azote stabilisé par parois (Fig. 16). Ces solutions montrent que l'enthalpie croît fortement dans la région d'entrée, atteint un pic puis décroît progressivement jusqu'à la région où l'arc est complètement développé (Fig. 16b). $\mathrm{Ce}$ qu'il convient de souligner tout particulièrement est que, en dépit de vitesses axiales élevées ( $1000 \mathrm{~m} / \mathrm{s})$ (Fig. 16a), l'écoulement du gaz à l'intérieur du constricteur (Fig. 16c) est essentiellement confiné dans une couche relativement froide (moins de $3000 \mathrm{~K}$ ) au voisinage des parois ce qui signifie que sur le débit total de gaz plasma en régime laminaire $50 \%$ à $70 \%$ du débit total n'est pas chauffé par le plasma. Ceci explique que les générateurs à

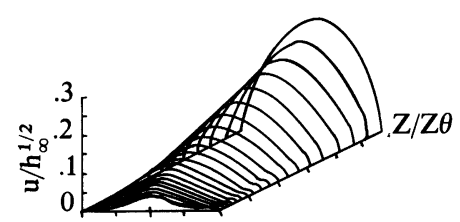

(a) Vitesse

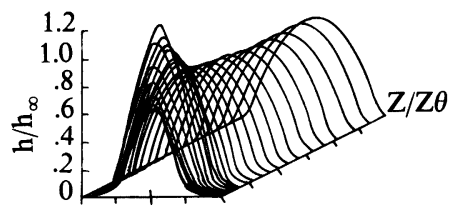

(b) Enthalpie

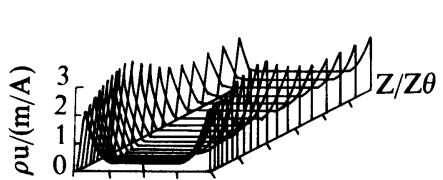

(c) Débit de gaz

$$
\begin{aligned}
\mathrm{I} & =580 \mathrm{~A} \quad \mathrm{Z} / \mathrm{Z} \theta \\
\mathrm{m} & =.00353 \mathrm{~kg} / \mathrm{s} \\
\mathrm{R} & =.00635 \mathrm{~m} \\
\mathrm{z}_{0} & =3.50 \mathrm{~m} \\
\mathrm{~h}_{\infty} & =1.89 \times 10^{8} \mathrm{~J} / \mathrm{kg} \\
\mathrm{m} / \mathrm{A} & =27.8 \mathrm{~kg} / \mathrm{sm}^{2} \\
\mathrm{~h}_{\infty} \mathrm{m} / \mathrm{A} & =5.29 \times 10^{9} \mathrm{~W} / \mathrm{m}^{2} \\
\mathrm{P}_{0} & =117 \mathrm{kPa}
\end{aligned}
$$

Fig. 16. - Modélisation par Waston et Pegot d'un écoulement plasma dans une tuyère [144] : a) Evolution des vitesses le long de l'axe. b) Evolution de l'enthalpie le long de l'axe. c) Evolution du débit de gaz le long de l'axe.

[Modelization by Waston and Pegot of a plasma flow inside a cooled walled nozzle : a) Evolution of the velocity distribution along the axis. b) Evolution of the enthalpy distribution along the axis. c) Evolution of the gas mass flowrate distribution along the axis.] injection tourbillonnaire aient été développés au plan industriel car il semble qu'ils permettent d'homogénéiser la température des jets et d'augmenter la masse de gaz réellement chauffée, à une température dans l'ensemble plus faible, mais largement suffisante pour la majorité des opérations recherchées.

Ces modèles laminaires sont cependant encore utilisés pour calculer les générateurs à plasma de puissance [145].

Naturellement de tels modèles n'ont de signification que si les résultats calculés peuvent être comparés aux mesures. Celles-ci sont cependant difficiles tant au plan fondamental que du fait des gradients élevés rencontrés et nécessitant un traitement mathématique complexe (inversion d'Abel des signaux obtenus). En ce qui concerne les températures, quelques dispositifs automatisés ont été mis au point, tant pour les températures d'excitation en balayant le jet, soit par déplacement avec des moteurs pas à pas [146], soit avec un miroir tournant [147], soit avec un diaphragme mobile [148], que pour les températures de rotation en utilisant notamment un tube amplificateur d'image permettant l'enregistrement simultanée de plusieurs spectres le long d'un rayon [149]. Pour les vitesses, malheureusement dans les générateurs atmosphériques $(v<1000 \mathrm{~m} / \mathrm{s})$ les méthodes de déplacement Doppler des raies d'émission ne sont pas utilisables et seule l'injection dans la chambre d'arc de particules fines dont la vitesse est alors mesurée par anémométrie laser permet d'accéder, avec difficulté et une précision douteuse dans le dard, à la vitesse du jet $[146,150]$. La comparaison de telles mesures effectuées à Limoges dans un générateur à plasma de projection [146] avec un modèle d'écoulement turbulent $[142,101]$ montre un accord raisonnable (cf. Figs. 17a et b tirées de [142]) et démontre notamment que de tels jets ne sont pas encore totalement turbulents mais en régime transitoire. Nous pouvons également souligner pour un arc avec une cathode de type FCC (Fluid Convective Cathode, cf. [112]) un assez bon accord entre les températures calculées en régime laminaire et celles mesurées (cf. Fig. 18 tirée de [136]).

3.1.2 Calculs hors équilibre. - Tous les modèles développés l'ont été pour les plasmas d'argon [37 à 42] en faisant apparaître une équation de l'énergie pour les particules lourdes et une pour les électrons. Connaissant la densité des électrons, les termes d'échange d'énergie en fonction de $T_{\mathrm{e}}$ et de $\theta$ $\left(\theta=T_{\mathrm{e}} / T_{\mathrm{h}}\right)$ soit

$$
E_{\mathrm{eh}}^{0}=\sum_{h} \frac{3}{2} k\left(T_{\mathrm{e}}-T_{\mathrm{h}}\right) n_{\mathrm{e}}\left(\frac{2 m_{\mathrm{e}}}{m_{\mathrm{h}}}\right) \bar{v}_{\mathrm{eh}}
$$

où $k$ est la constante de Boltzmann, $\bar{v}_{\text {eh }}$ la fréquence de collision moyenne entre électrons et particules lourdes (le rapport $2 m_{\mathrm{e}} / m_{\mathrm{h}}$ traduisant l'échange d'énergie d'une collision élastique), il est possible de résoudre les équations de conservations et de déterminer les champs de température $\left(T_{\mathrm{e}}, T_{\mathrm{h}}\right)$ et d'écou- 

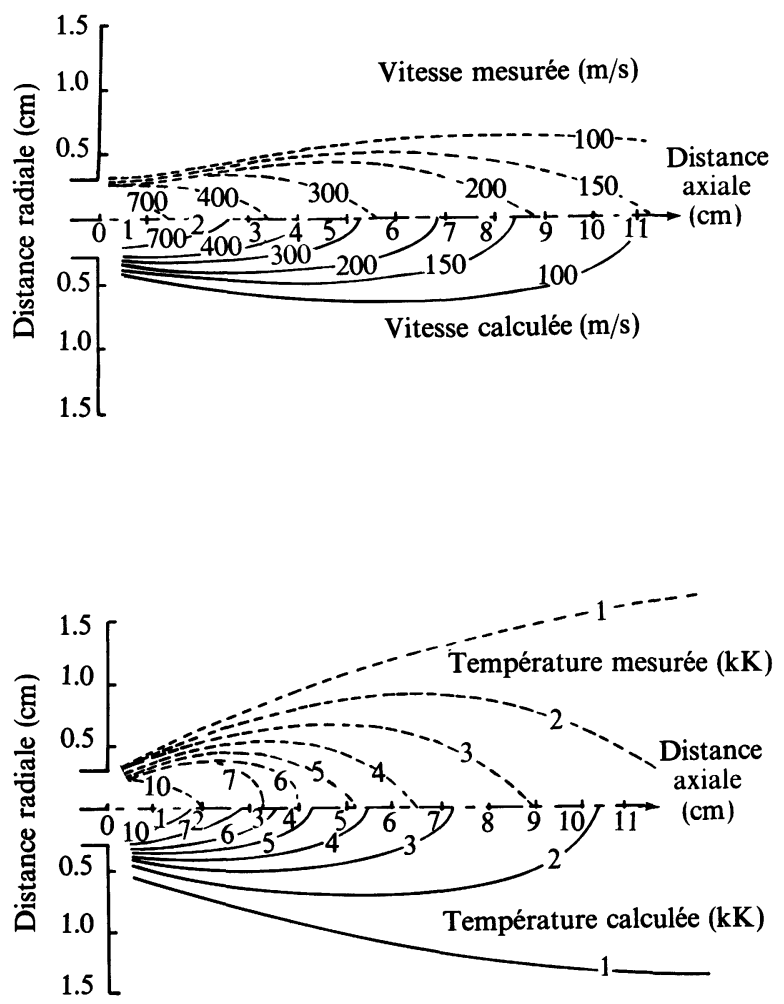

Fig. 17. - Comparaison des vitesses et des températures calculées et mesurées à la sortie de la tuyère du générateur représenté figure 6 [142].

[Comparison of the calculated and measured velocity and temperature distributions after the nozzle exit of the plasma generator represented figure $6\left(\mathrm{Ar}-\mathrm{H}_{2}\right.$ plasma $\left.29 \mathrm{~kW}\right)[142]$.]

lement. Ces calculs montrent notamment que les plasmas d'arc sont hors d'équilibre $(1<\theta<2)$ au voisinage des parois, sur les bords des jets ou lors de l'injection d'un gaz froid.

3.2 LES RÉACTIONS CHIMIQUES. - Un écoulement plasma est très complexe, fortement inhomogène avec des gradients considérables et enfin très visqueux. Un tel milieu sans réactions chimiques est donc déjà extrêmement difficile à modéliser. Si l'on veut effectuer des réactions chimiques, en plasma, il convient, si le gaz lui-même est déjà réactif (mélange homogène des réactifs effectué avant la génération du plasma) d'introduire les effets cinétiques et si le réactif froid doit être introduit dans le plasma (par exemple après les électrodes dans un arc à cathode chaude) d'étudier simultanément le mélange des gaz froids avec le plasma et les réactions chimiques induites lors de ce mélange. A notre connaissance, un tel problème n'a encore jamais été résolu et la situation actuelle est la suivante :

- des études d'écoulement lors de l'injection d'un gaz froid non réactif dans le plasma (argon dans argon) ont juste débuté,

- des études des réactions cinétiques en fonction de la température et avec des hypothèses très simplificatrices (par exemple la diffusion est négligée, le plasma est supposé homogène radialement...) en sont également à leurs premiers balbutiements.

Nous allons donc sommairement résumer ces deux situations.

3.2.1 Les études cinétiques. - L'équilibre dépend du paramètre de Damköhler [151] $D_{i}=t_{\mathrm{p}} / t_{\mathrm{m}}$ où $t_{\mathrm{p}}$ est un temps de relaxation caractéristique des réactions mises en jeu et $t_{\mathrm{m}}$ le temps de résidence des réactifs dans une zone déterminée à $T$ donnée : les calculs sont simples, si $D_{i} \ll 1$, car l'écoulement est à l'équilibre ou si $D_{i} \gg 1$, car l'écoulement est figé. Malheureusement dans le cas des plasmas thermiques $D_{i} \sim 1$ et il faut donc prendre en compte les équations cinétiques.

Outre les problèmes de résolution de ces systèmes d'équations différentielles non linéaires couplées, la difficulté principale est la détermination des constantes de réaction $k_{j}$ pour lesquelles on utilise à la fois des calculs théoriques [153 à 157] et des valeurs expérimentales. Une telle approche ne conduit pas à des valeurs de $k$ très précises [158] et les valeurs de $k$ de la littérature sont parfois différentes pour une même réaction suivant les hypothèses de calculs ou les dispositifs expérimentaux utilisés. Enfin, au-delà de $3000 \mathrm{~K}$ il n'y a pratiquement pas de résultats expérimentaux. De nombreuses études fondamentales couplées à des expériences (par exemple via la fluorescence laser) sont donc encore nécessaires pour résoudre ce problème des données.

Les principaux calculs utilisant l'équation donnant l'évolution temporelle de la composition ont été effectués pour les plasmas basse pression dans des gaz purs dont on suit l'évolution des espèces excitées

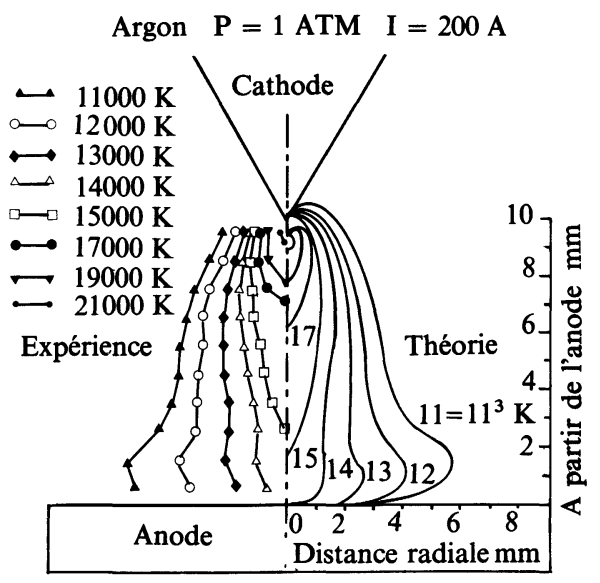

Fig. 18. - Comparaison des températures calculées et mesurées dans un arc à cathode convective $(I=200 \mathrm{~A}$, distance anode - cathode $=10 \mathrm{~mm}$ gas plasma argon) [136].

[Comparison of the calculated and measured temperature distribution of an arc with a fluid convective cathode $(I=200 \mathrm{~A}$, distance between the cathode tip and the anode, $10 \mathrm{~mm}$, plasma gas : argon) [136].] 
avec le temps lorsque l'on coupe la décharge (flowing afterglow) comme par exemple dans une décharge d'oxygène [159] ou pour les phénomènes de dissociation [160]. Toutefois, peu de développements ont été faits jusqu'alors dans les plasmas thermiques si ce n'est pour évaluer les effets de trempe : Polak [161], Zel'Dovich [162], Duff [163], Amman [164]. Les plus récents travaux dans le domaine sont ceux du Laboratoire successivement sur la cinétique de production des oxydes d'azote en plasma d'arc [165 à 170] et sur la réduction du tétrachlorure de silicium [171].

Quoique simplifiés, en utilisant par exemple une température enthalpique moyenne pour le plasma, ces calculs ont permis d'expliquer pourquoi le taux de conversion de l'azote en oxyde, dans un plasma d'arc fonctionnant à l'azote et où on injecte de l'oxygène froid, est supérieur $(12 \%)$ à celui prévu par l'équilibre $(6,5 \%)$ du fait des réactions moléculaires entre états vibrationnellement excités [169-170]. Il en va de même pour la production de silicium à partir de $\mathrm{SiCl}_{4}$ où l'hydrogène joue le rôle de piège à chlore [171] comme le montre la figure 19 représentant l'évolution des différentes espèces lors du chauffage et de la trempe (suivant une loi $T=f(t)$ du mélange $\mathrm{SiCl}_{4}+\mathrm{H}_{2}$ ), dans un générateur -à plasma d'arc $\left(\mathrm{SiCl}_{4}\right.$ injecté à la sortie de la tuyère du générateur fonctionnant à l'hydrogène).

Tout ceci correspond bien entendu aux réactions en phase homogène et pour être complet, il conviendra de parler de l'influence des parois sur les réactions, ce qui rejoint la catalyse en plasma [172 à 188].

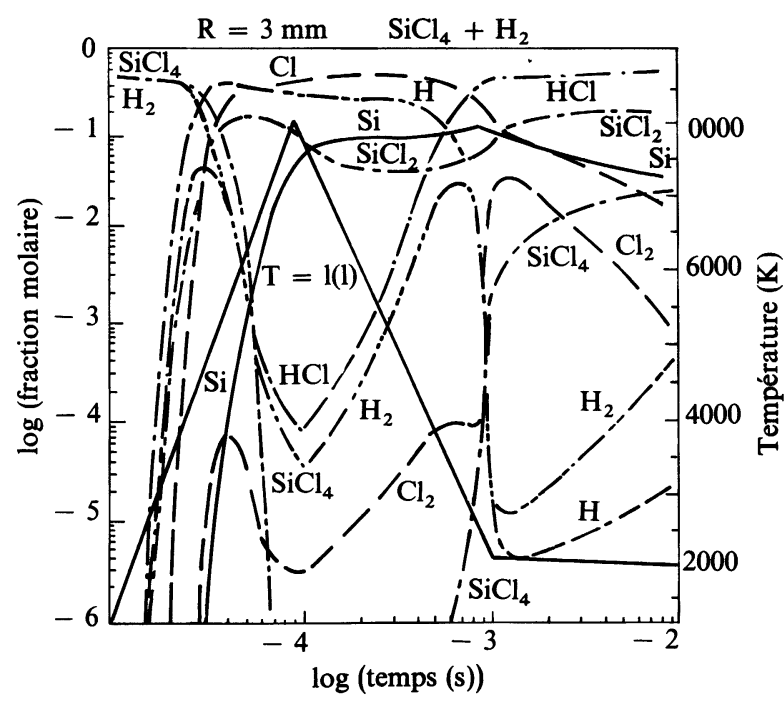

Fig. 19. - Cinétique de la réaction $\mathrm{SiCl}_{4}+\mathrm{H}_{2}$ lorsque ce mélange est soumis à une loi de chauffage et de trempe $T=f(t)$ avec une température maximale de $6000 \mathrm{~K}$ : évolution en fonction du temps du logarithme des fractions molaires des différentes espèces formées [171].

[Kinetic of the reaction $\mathrm{SiCl}_{4}+\mathrm{H}_{2}$ when this mixture is heated up to $6000 \mathrm{~K}$ and quenched following a law $T=f(t)$ : evolution with time of the logarithm of the molar fraction of the different species formed [171].]
Les travaux de l'équipe Amouroux [173, 182 à 185, 189] tant sur les synthèses de NO et HCN que sur la décomposition de $\mathrm{NH}_{3}$ dans des plasmas $\mathrm{HF}$ à basse pression (quelques torrs) ont permis de dégager un phénomène de catalyse commun : à savoir un processus de chimisorption à partir des états de faible énergie. Tous ces travaux ont montré qu'en présence de catalyseurs on pouvait obtenir des taux de conversion très supérieurs à ceux obtenus en leur absence, comme par exemple : $17 \%$ d'azote fixé sous forme de $\mathrm{NO}$ avec $\mathrm{WO}_{3}$ comme catalyseur contre $8 \%$ sans catalyseur, $4,6 \%$ d'azote fixé sous forme de $\mathrm{HCN}$ dans un plasma d'azote où l'on injecte du méthane contre $0,9 \%$ sans catalyseur. De récentes études au laboratoire et chez Amouroux [189] montrent d'ailleurs que de tels travaux peuvent être étendus à la pression atmosphérique.

3.2.2 Problème du mélange d'un gaz froid avec le plasma. - Dans le cas d'un système de symétrie cylindrique, l'injection, en symétrie axiale, d'un gaz froid dans un plasma se traduit simplement par l'écriture des conditions aux limites appropriées. Les rares calculs développés sont ceux de Pfender et ses collaborateurs $[103,102]$ pour les arcs avec injection perpendiculaire de gaz froid et ceux de Boulos et ses collaborateurs [190,191] pour les plasmas HF avec soit injection parallèle (protection des parois) [190], soit perpendiculaire [191].

Par exemple, Pfender [102] par l'injection en aval de l'anode, d'argon froid dans un plasma d'argon produit par arc électrique montre à l'ETL que cette injection a tendance à constricter les isothermes et que le gaz froid ne pénètre pas au coeur du jet de plasma mais passe en périphérie du jet, limitant le transfert de chaleur vers les parois (le mélange ne commence vraiment à se faire qu'après une distance d'environ 5 à 6 fois le diamètre du canal après la fente d'injection). On conçoit donc que de tels effets puissent sérieusement affecter les réactions chimiques en plasma et qu'il convient de développer ces études.

Le modèle à deux températures de Pfender [103] dans les mêmes conditions montre que dans la région voisine de la fente d'injection, la température des électrons est nettement plus élevée que celle des particules lourdes (Fig. 20).

3.3 LeS TRANSFERTS DE CHALEUR ET DE QUANTITÉ DE MOUVEMENT PLASMA-PARTICULES. - Le traitement des matériaux dans les plasmas thermiques est conditionné par le transfert thermique plasma-particules comme cela a été montré pour la projection d'alumine [192] ou pour l'obtention de particules sphériques [193]. Malheureusement, du fait des gradients et des valeurs des températures et des vitesses rencontrées, l'étude de ces transferts est très difficile, tant au plan théorique qu'expérimental [194] et il n'existe pas de solution générale, même approximative à ces problèmes. En effet, la présence des particules modifie l'écoulement plasma [195], elles peuvent 


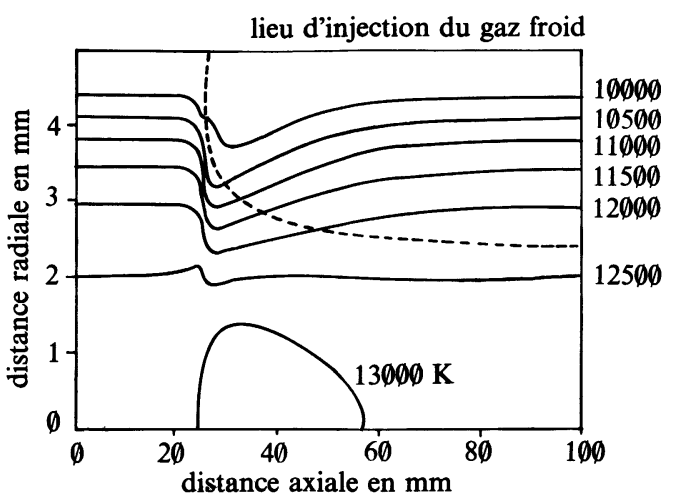

Fig. 20a

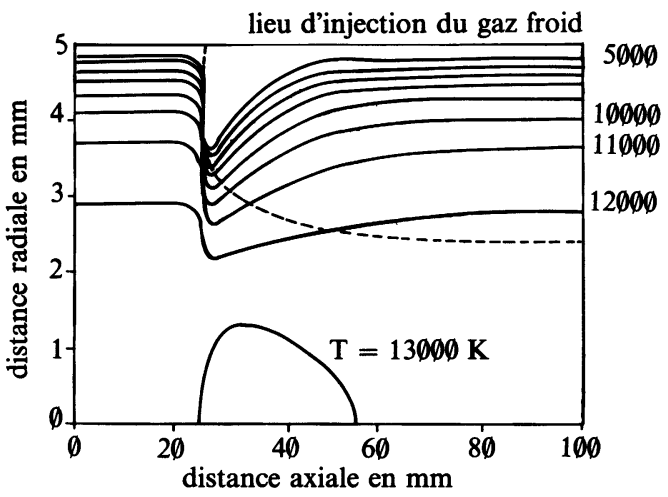

Fig. 20b

Fig. 20. - a) Distribution de la température des électrons dans un générateur à arc continu à l'argon $(p=1 \mathrm{~atm}$, $\left.I=200 \mathrm{~A}, \quad m_{\mathrm{Ar}}^{\circ}=0,357 \mathrm{~g} / \mathrm{s}\right)$ dans lequel on injecte de l'argon froid $\left(m_{\mathrm{Ar}}^{\circ}=0,165 \mathrm{~g} / \mathrm{s}\right)$; la ligne en pointillé correspond à la pénétration maximale de l'écoulement froid [103]. b) Distribution de la température des lourds.

[a) Electron temperature distribution inside the nozzle of a de plasma torch (argon at $1 \mathrm{~atm}, I=200 \mathrm{~A}, m_{\mathrm{Ar}}^{\circ}=0.357 \mathrm{~g} / \mathrm{s}$ ) when a cold argon gas is symetrically injected $\left(m_{\mathrm{Ar}}^{\circ}=\right.$ $0.165 \mathrm{~g} / \mathrm{s}$ ), the dotted line corresponds to the maximum penetration of the cold flow. b) Heavy particles temperature distribution under the same conditions [103].]

avoir entre elles et avec les parois une interaction non négligeable, les propriétés thermodynamiques et thermiques du plasma varient considérablement avec la température et les gradients de température et de vitesse du plasma sont élevés. Il y a donc couplage entre les distributions de température et de vitesse pour le plasma et les particules. Deux approches sont alors possibles :

- considérer les particules comme isolées [196] et suivre exactement leur trajectoire dans le plasma,

- aborder l'évolution globale des particules dans la direction de l'écoulement $[197,198]$ au détriment de la description locale du système.

Bonet [194] a montré que dans la majorité des cas d'utilisation industrielle des plasmas, du fait des temps de résidence très brefs des particules, on pouvait en fait considérer l'ensemble plasma-particules comme un milieu très dilué. Dans ces conditions, les interactions entre les particules sont généralement négligeables comme l'a montré Mathieu [199] pourvu qu'une certaine inégalité soit satisfaite, ce qui est d'ailleurs le cas de la majorité des applications actuelles. Cependant lors de la vaporisation des particules, la modification de la température du plasma (chauffage de la vapeur) n'est pas négligeable, comme l'ont montré récemment Proulx et al. [195].

3.3.1 Les transferts de quantité de mouvement. Lorsque l'on connaît la distribution des vitesses et des températures de l'écoulement, si possible modifiée pour tenir compte de l'injection du gaz porteur froid (cf. §3.2.2), il est possible de déterminer la trajectoire d'une particule en utilisant l'équation de Basset-Boussinesq-Oseen donnant la force agissant sur la particule [200 à 203]. Ces équations sont raisonnablement simples à résoudre tant qu'il n'y a pas de couplage avec les équations de l'énergie (absence de vaporisation).

Un des points essentiels est la détermination du coefficient de traînée. Ce coefficient est calculé en fonction du nombre de Reynolds [196, 202] pour des particules de forme quelconque ou sphérique, formules déduites de données expérimentales de Pruppacher [204]. Cependant, la difficulté qui se pose est de savoir quelles valeurs utiliser pour les propriétés de transport : celles du plasma loin de la particule, celles de la température de surface de la particule, des valeurs intermédiaires ? Même dans le cas simple d'un plasma d'argon Lewis et Gauvin [205], ou Pfender et al. [102] suggèrent des solutions relativement très différentes pour tenir compte des gradients thermiques considérables rencontrés dans la couche limite entourant la particule.

De plus lorsque la taille des particules devient comparable devant le libre parcours moyen (ce qui est le cas lorsque leur diamètre est inférieur à $10 \mu \mathrm{m}$ ), il convient de tenir compte des effets de raréfaction et de multiplier le coefficient de traînée par un terme correctif [206]. La prise en compte d'un tel terme peut alors modifier assez sensiblement la trajectoire des particules [206].

Un terme éventuellement susceptible d'avoir de l'importance est le terme des forces de thermophorèse qui s'exercent sur les particules (principalement celles dont le diamètre est inférieur à $20 \mu \mathrm{m}$ ) lors de leur pénétration dans le plasma du fait de l'existence d'importants gradients thermiques [203]. Le problème là aussi est la valeur à donner aux coefficients qui ont généralement été évalués pour des gradients inférieurs à $200 \mathrm{~K} / \mathrm{mm}$ alors que l'on rencontre jusqu'à $4000 \mathrm{~K} / \mathrm{mm}$ dans les plasmas ! Certains calculs [207] indiquent que ces forces sont négligeables pour des particules dont le diamètre est supérieur à $10 \mu \mathrm{m}$, résultat qui est cependant sujet à caution [104] dans la mesure par exemple où des particules d'alu- 
mine de $20 \mu \mathrm{m}$ de diamètre ayant le double de la vitesse théorique nécessaire pour avoir une trajectoire axiale après leur injection dans un générateur de projection n'atteignent cet axe que pratiquement hors du dard du jet de plasma. Toutefois l'influence du gaz porteur sur la modification des propriétés du plasma (phénomènes de striction) n'est pas clairement établie et il est difficile d'estimer ces forces de thermophorèse.

Des calculs de transfert de quantité de mouvement plasma-particules ont été effectués aussi bien dans les plasmas HF [190, 208] que dans les arcs [102, 209 à 212]. Ils mettent en évidence la diversité des trajectoires suivies selon le lieu et la vitesse d'injection, cependant sauf les calculs de Pfender [102], aucun ne prend en compte la perturbation de l'écoulement par le gaz porteur. Les comparaisons avec les mesures sont rares $[210,206,213]$, mais elles semblent cependant indiquer que les valeurs utilisées des coefficients de traînée sont une assez bonne approximation comme le montre par exemple la figure 21 où est représentée l'évolution des vitesses le long de l'axe d'un jet de plasma de projection de particules d'alumine de différents diamètres. On constate bien sûr que les particules suivent d'autant mieux la vitesse du gaz qu'elles sont petites. Cependant même les particules de $50 \mu \mathrm{m}$ de diamètre atteignent des vitesses de plus de $100 \mathrm{~m} / \mathrm{s}$ ce qui signifie des temps de séjour, dans des jets de moins de $100 \mathrm{~mm}$ de long,

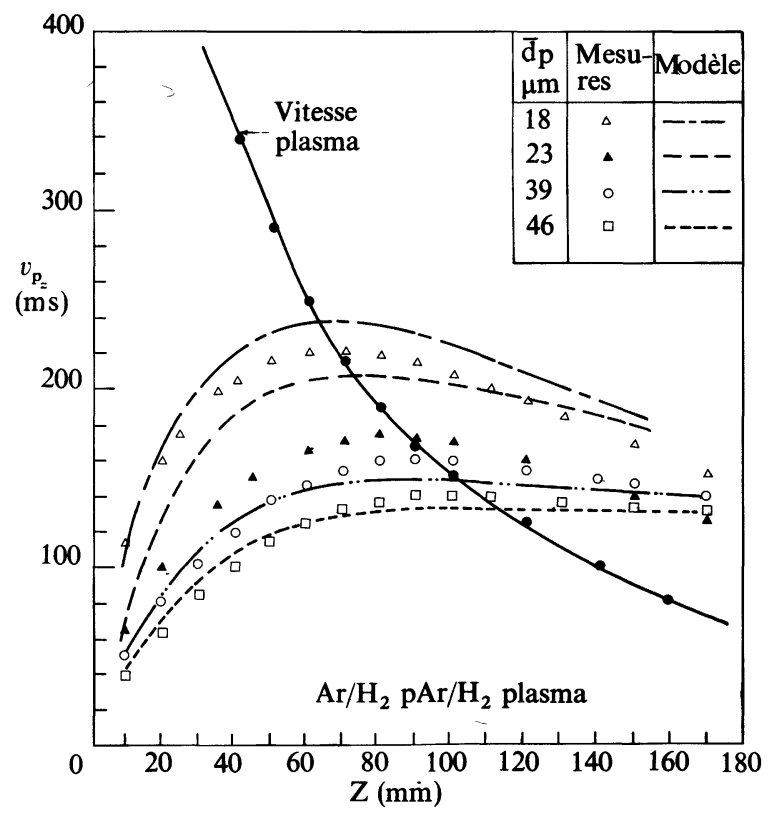

Fig. 21. - Evolution le long de l'axe d'un jet de plasma $\left(\mathrm{Ar}-\mathrm{H}_{2}\right)$ utilisé en projection (cf. Fig. 6) de la vitesse de particules d'alumine de différents diamètres : courbes calculées, points mesurés [210].

[Evolution, along the axis of a plasma jet $\mathrm{Ar}-\mathrm{H}_{2}$ used for spraying (see Fig. 6) of the velocity of alumina particles with different diameters : lines : calculated curves, points : measured values [210].] inférieurs à la milliseconde. Des temps un peu supérieurs (de l'ordre de 10 à $100 \mathrm{~ms}$ ) ont été calculés pour des générateurs du type de calcul de Westinghouse [214] utilisés industriellement.

3.3.2 Les transferts de chaleur plasma-particules. Au cours des dernières annees, de tres nombreux modèles mathématiques ont été développés pour le calcul des transferts de chaleur plasma-particules. La quasitotalité sont des modèles ne prenant pas en compte l'interaction des particules entre elles et les modifications entraînées par leur introduction dans le jet de plasma, ce sont, soit des modèles à une dimension, plus ou moins simples : Bonet [194] (1974), Yoshida [208] (1977) et Gal-Or [215] (1980), soit des modèles à deux dimensions : Boulos [196] (1974) et [190] (1978), Battacharyya et Gauvin [202] (1975), Fiszdon [211] (1975) et [212] (1979). Un seul modèle ultra simplifié tente de prendre en compte les interactions entre les particules ou plus précisément de déterminer dans quelles conditions on peut les négliger, c'est celui de Mathieu [199] (1979). Enfin comme nous l'avons déjà indiqué, le modèle de Proulx et al. [195] tente de prendre en compte les effets de vaporisation sur le plasma.

a) Les coefficients d'échange. - Dans le cas de la conduction, les travaux de Sayegh et Gauvin [216] ont permis d'établir des relations complexes pour le coefficient de Nusselt conductif $(\mathrm{Nu})$. Cependant, Bourdin [217] a démontré que l'on pouvait utiliser $N u=2 \mathrm{si}$ l'on utilise la valeur intégrée moyenne de la conductivité thermique $\kappa$ entre la température de surface de la particule et la température du plasma non perturbé. Il est alors important de noter la variation brutale de $\bar{\kappa}$ avec la température lors de la dissociation qui est due à la conductivité thermique réactionnelle qui varie brutalement à la température de dissociation $T_{D}$. Le meilleur transfert thermique sera donc obtenu pour des gaz à faible température de dissociation comme l'hydrogène ainsi que le montre la figure 22 représentant l'évolution de $\bar{\kappa}$ avec la température pour un plasma à la pression atmosphérique en ETL. Ceci explique l'utilisation de l'hydrogène ou de gaz diatomiques tels que $\mathrm{CO}$, l'air, voire polyatomiques comme la vapeur d'eau dans tous les plasmas ou les transferts thermiques sur des particules ou des solides sont importants (projection ou chauffage).

En l'absence de réactions chimiques, le terme convectif du coefficient de Nusselt est généralement exprimé en fonction des nombres de Reynolds et Prandlt [201, 202, 216], suivant la corrélation de Ranz et Marshall [218] établie pour des gouttelettes. Dans tous les cas le terme convectif du coefficient de Nusselt évolue entre 0,1 et 0,7 contre 2 pour le conductif.

Comme pour le coefficient de traînée, Pfender [102] suggère également de corriger le coefficient de Nusselt comme le coefficient de traînée en faisant intervenir des expressions complexes avec la masse spécifique, la viscosité, la chaleur spécifique. 


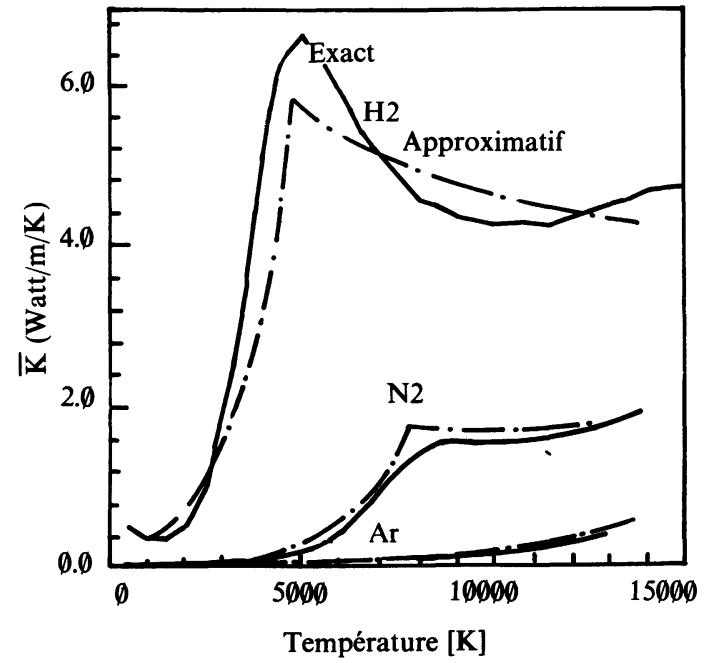

Fig. 22. - Evolution avec la température de la conductivité thermique moyenne intégrée $\bar{\kappa}$ de différents gaz plasma à la pression atmosphérique [217] :

$$
\bar{\kappa}=\frac{1}{T-300} \int_{300}^{T} \kappa(t) \cdot \mathrm{d} t .
$$

[Evolution with temperature of the mean integrated thermal conductivity for various plasma gases at atmospheric pressure [217].]

Enfin, pour une particule isolée, Bourdin [217] a montré que les pertes par rayonnement doivent être prises en compte uniquement pour un plasma d'argon ou d'azote à $T<4000 \mathrm{~K}$ avec des particules dont la surface dépasse $2000 \mathrm{~K}$.

Pour l'étude du rayonnement, des modèles multiparticulaires unidimensionnels ont également été développés [199, 219] qui montrent que pour la majorité des applications du plasma les interactions entre particules sont généralement négligeables.

b) Quelques phénomènes à prendre en compte. Pour la modification des propriétés de la couche lors de l'évaporation de la particule, l'hypothèse la plus simple consiste à supposer que le flux de vapeur issu de la particule se mélange au plasma qui l'entraîne modifiant ainsi les propriétés de transport et Yoshida [208] suggère de les calculer à partir d'équations simplifiées. Ces nouvelles propriétés de transport sont alors utilisées pour calculer les coefficients de Nusselt et de traînée. De tels calculs ont par exemple été développés récemment par Dinulescu [220] pour des plasmas d'argon en présence de vapeurs métalliques.

De plus ce flux d'évaporation, opposé au flux de chaleur venant du plasma peut réduire notablement le coefficient Nusselt comme l'ont montré Proulx et al. [195] puisque dans un plasma d'argon au voisinage de $15000 \mathrm{~K}$ (température pour laquelle le transfert est maximal dans l'argon) cette réduction peut atteindre $40 \%$ pour des particules d'alumine. Il en va de même de l'effet de raréfaction pour les particules de diamètre inférieur à $10 \mu \mathrm{m}[221,222,195]$, où toujours pour des particules d'alumine, il a été montré [195] que la diminution du transfert de chaleur était de plus de $50 \%$ pour des particules de $2 \mu \mathrm{m}$ de diamètre. Ces effets, conjugués avec les phénomènes de propagation de la chaleur (dans un plasma $\mathrm{Ar}-\mathrm{H}_{2}$ une particule commence à se vaporiser en surface alors que son centre n'a pas dépassé $500 \mathrm{~K}$ [217]) expliquent qu'il est possible d'injecter des particules d'alumine de 2 à $3 \mu \mathrm{m}$ de diamètre dans la chambre d'arc et de les suivre par anémométrie laser à la sortie de la tuyère pour mesurer la vitesse du plasma (sans la prise en compte de ces effets, le calcul indique que de telles particules sont vaporisées en quelques millimètres).

Bonet [194] a tenu compte de l'effet Soret, de la diffusion Fick, de la thermodiffusion (effet Dufour) et de la diffusion convective. Ses calculs, pour des particules de $\mathrm{ZrO}_{2}$, montrent que la correction due à la thermodiffusion est négligeable et que la diffusion enthalpique couplée à l'effet Soret est faible mais non négligeable au-delà de $3200 \mathrm{~K}$ pour les particules.

Le phénomène de propagation de la chaleur dans la particule doit être pris en compte [217] dès lors que le rapport : $\bar{\kappa} / \kappa_{\mathrm{p}}>0,03$ (où $\bar{\kappa}$ est la conductivité thermique intégrée et $\kappa_{\mathrm{p}}$ celle de la particule) comme on peut le voir sur la figure 23, représentant l'évolution de la température de surface et de la température du centre d'une particule d'alumine immergée dans un plasma d'azote à $10000 \mathrm{~K}$.

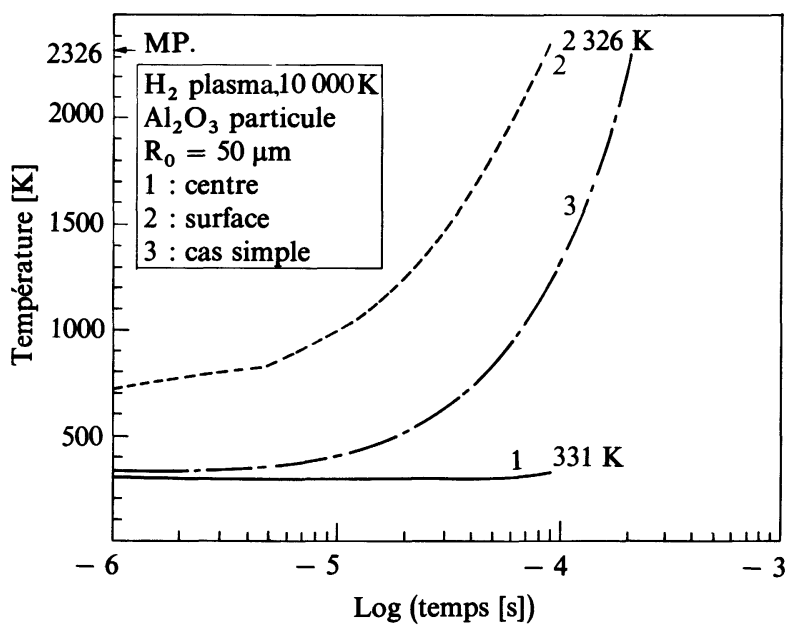

Fig. 23. - Evolution de la température de la surface (courbe 2) et de la température du centre (courbe 1) d'une particule d'alumine de $100 \mu \mathrm{m}$ de diamètre immergée dans un plasma d'azote à $10000 \mathrm{~K}$ (le rapport de la conductivité thermique moyenne intégrée du plasma à la conductivité thermique de la particule est égal à 0,3 ). La courbe 3 représente l'évolution de la température de la particule lorsque les phénomènes de propagation ne sont pas pris en compte [217].

[Evolution with time of the surface temperature (curve 2) and of the centre temperature (curve 1) of a $100 \mu \mathrm{m}$ diameter alumina particle immersed in an infinite nitrogen atmospheric plasma at $10000 \mathrm{~K}\left(\kappa / \kappa_{\mathrm{p}} \sim 0.3, \kappa_{\mathrm{p}}\right.$ being the thermal conductivity of the particle). Curve 3 represents the evolution of the particle temperature when its thermal conductivity is supposed to be infinite (no heat propagation inside the particle) [217].] 
Ce qu'il convient alors de noter, est que, même en utilisant un plasma d'hydrogène pour lequel le transfert thermique est excellent, il faut [217, 223] près d'une milliseconde pour que la température de surface d'une particule d'alumine de $200 \mu \mathrm{m}$ de diamètre atteigne la température de fusion (alors que le centre de la particule est toujours froid) et plus d'une milliseconde pour qu'elle commence à se vaporiser en surface, cette vaporisation sans réactions chimiques étant achevée en plus d'une seconde.

c) Comparaison des calculs aux expériences. Très peu de mesures, du fait des difficultés expérimentales [146], ont été effectuées à notre connaissance sinon celles de Fizdon [211] et celles de Vardelle [210] sur des particules d'alumine. Elles mettent en évidence un écart important entre la température de surface calculée et celle mesurée (près de $500 \mathrm{~K}$ ). Les raisons de cet écart, d'ailleurs confirmé lors de la projection d'alumine [224] par les quelques pourcentages d'alumine $\alpha$ restant dans le dépôt en phase $\gamma$ et qui montrent bien que la fusion, contrairement aux calculs, n'est pas complète, peuvent être les suivantes :

- perturbation et refroidissement du plasma par le gaz porteur (négligés par les calculs),

- importance des forces de thermophorèse dont les coefficients, mesurés dans des gaz chauds, ne sont certainement pas transposables au plasma. Ce dernier point est peut-être corroboré par de récentes mesures $[210,225]$ qui montrent que les particules ne pénètrent pas vraiment dans les zones chaudes du jet, même avec une vitesse d'injection double de celle calculée théoriquement,

- l'équilibre thermodynamique local est-il bien réalisé au voisinage de la particule,

- les effets d'évaporation et raréfaction n'ont pas été pris en compte...

3.3.3 Les réactions chimiques en phase hétérogène. Depuis les travaux de Bonet [226] et ceux de Munz [227], pratiquement rien n'a été fait. Les réactions sont en fait contrôlées par les phénomènes de transfert thermique d'une part et les phénomènes de diffusion d'autre part. Naturellement, la réaction est alors contrôlée par le plus lent des deux processus. Compte tenu de la complexité des phénomènes mis en jeu et du manque de données, il est peu probable que des résultats autres que qualitatifs, comme par exemple pour la décomposition de la molybdénite [227], puissent être obtenus. Toutefois il faut souligner que ces résultats, ainsi que des calculs menés au laboratoire sur la réduction des oxydes de fer, montrent que les temps moyens de réaction se situent au voisinage de la seconde pour des particules d'une cinquantaine de microns de diamètre.

3.4 LES POINTS ESSENTIELS QUI SE DÉGAGENT DE CETTE MODÉLISATION. - Certes ces modélisations des écoulements plasma, des réactions chimiques et des traitements des particules solides en plasma n'en sont encore qu'à leur tout début, cependant, elles soulignent plu- sieurs points dont il doit être tenu compte pour la réalisation technologique des réacteurs à plasma :

- seule une fraction des gaz plasmas (parfois inférieure à $50 \%$ ) est effectivement portée à haute température, le reste du gaz plasmagène passant en périphérie et restant à une température inférieure à $3000 \mathrm{~K}$, l'utilisation de générateurs à injection tourbillonnaire augmentant la fraction de gaz chauffée,

- l'injection d'un gaz froid dans le plasma est relativement difficile et le gaz froid pénètre mal et reste en périphérie des gaz chauds sur des longueurs importantes. De plus dans la zone d'injection le plasma devient hors équilibre avec des températures électroniques de l'ordre du double des températures des particules lourdes. Enfin dans ces zones d'injection le jet est constricté rendant la pénétration encore plus difficile,

- du fait de cette pénétration difficile des gaz froids réactifs (il n'est pas toujours possible de mettre des gaz réactifs tels l'oxygène ou les chlorures en contact avec les électrodes), la majorité des réactions s'effectue dans des zones relativement froides et on a essentiellement une chimie entre états moléculaires excités, chimie encore extrêmement mal connue (avec des réactions ayant lieu en moins d'une microseconde) mais très prometteuse comme le montrent par exemple les expériences sur les oxydes d'azote (taux de réactions supérieurs de deux fois à ceux de l'équilibre). Il semblerait donc utile de développer des générateurs ayant des températures moyennes nettement plus faibles (inférieures à 6-7000 $\mathrm{K}$ par exemple) où le mélange se ferait plus facilement et où les réactions entre états moléculaires excités auraient toujours lieu. Enfin étant donné les vitesses de passage dans l'arc et les vitesses d'expansion des jets de plasma en extinction, les vitesses de chauffage peuvent atteindre $10^{9} \mathrm{~K} / \mathrm{s}$ et celles de trempe $10^{8} \mathrm{~K} / \mathrm{s}$, ce qui signifie que l'on est en présence d'une chimie totalement hors équilibre et encore très mal connue,

- la pénétration des particules solides dans un plasma est généralement difficile et conditionnée par des phénomènes encore mal connus comme l'effet du gaz porteur sur la striction des jets où l'influence de certaines forces (thermophorèses). De plus pour les petites particules (inférieures à $10 \mu \mathrm{m}$ ) les effets de vaporisation et de raréfaction peuvent être importants,

- les transferts de chaleur plasma-particules sont d'autant meilleurs que le gaz plasma est diatomique ou polyatomique, l'hydrogène et la vapeur d'eau donnant les transferts les plus efficients si la température du plasma est supérieure à la température de dissociation. Ceci implique également de rechercher de nouvelles technologies de générateur donnant des jets plus homogènes à des températures légèrement supérieures aux températures de dissociation, l'utilisation de températures plus élevées n'améliorant pratiquement pas le transfert de chaleur,

- enfin, même avec des plasmas très efficients comme l'hydrogène, il convient de retenir les temps 
caractéristiques suivants pour une particule céramique de $50 \mu \mathrm{m}$ de diamètre : fusion $\sim 1 \mathrm{~ms}$ - vaporisation $\sim 100 \mathrm{~ms}$ - réaction chimique $\sim 1 \mathrm{~s}$ (dans l'hypothèse où la réaction a lieu à une température telle que la vaporisation est faible). De telles valeurs impliquent donc que les générateurs à cathodes thermoémissives où les vitesses d'écoulement sont élevées soient essentiellement utilisées pour la projection (il suffit de la fusion des particules), que les générateurs à électrodes cuivre tubulaires (générateurs à plasma de puissance) puissent à la limite être utilisés pour la vaporisation de métaux et que seuls les fours à plasma permettent les réactions chimiques en phase hétérogène.

\section{Applications actuelles et potentielles.}

4.1 InTRODUCTION GÉNÉRALE. - Après de longues années de recherche et de développement, la technologie plasma pénètre dans l'ère d'industrialisation pour des domaines d'application encore très largement ouverts, en chimie notamment. L'on dispose maintenant de torches de production de plasma industrielles, dont le fonctionnement est fiable, comme l'a démontré leur utilisation dans des processus industriels dépassant largement le stade du prototype en métallurgie.

Le développement et les recherches s'orientent notamment vers l'étude des procédés et non plus seulement vers celle du plasma seul. Les avantages de ces procédés :

- température élevée des gaz,

- concentration de la chaleur,

- séparation de la source de chaleur (électricité) et des gaz plasma,

- génération d'une atmosphère à la demande, quasi indépendante des réactions en cours (par exemple indépendance du potentiel d'oxygène),

- facilité de fonctionnement, de mise en route et d'arrêt,

ne peuvent être exploités pleinement que dans le cadre d'une génération du plasma adaptée à un processus de traitement donné. Les premières études technicoéconomiques publiées comme celles de la production du ferro-molybdène [228], montrent que si le réacteur à plasma peut être le cour du procédé, il reste généralement une petite partie de l'ensemble et qu'un programme de développement industriel doit nécessairement prendre en compte les coûts totaux d'investissement et de fonctionnement de l'unité de production complète. Dans de telles évaluations, le coût du plasma proprement dit apparaît souvent comme une part modeste.

Sans vouloir reprendre de récentes études générales $[229,230]$ présentant quelques applications actuelles et potentielles des plasmas thermiques, nous allons tenter d'indiquer ici quelles sont les grandes lignes de développement des fours plasmas dans l'industrie en nous limitant aux applications susceptibles de développements à une grande échelle de puissance comme la fusion ou la refusion, la sphéroïdisation, le séchage, la métallurgie extractive, la production de gaz réducteurs et la chimie des petites molécules (NO, $\mathrm{C}_{2} \mathrm{H}_{2}$, $\mathrm{HCN}$ ) en tentant de bien situer les délais de pénétration (actuel, court, moyen ou long terme).

4. 2 Les traitements en PhaSe GazeUSE. - Plusieurs types d'utilisation sont possibles :

- le chauffage dès lors que le gaz plasma est bon marché comme l'air ou la vapeur d'eau,

- la production de gaz réducteurs pour des opérations de métallurgie,

- les réactions chimiques en phase gazeuse ou à partir de produits assez facilement volatilisables comme le charbon ou les hydrocarbures lourds.

4.2.1 Chauffage. - Cette application n'est encore que prospective car jusqu'alors peu de fabricants étaient susceptibles de fournir des réacteurs à air fiables avec des durées de vie d'électrode compatibles avec un usage industriel. Cependant dès maintenant, avec des réacteurs du type de ceux de Westinghouse [58, 231, 232], de la SNIAS [233], de Tech. Appl. Service [234], le chauffage avec des plasmas d'air est possible. Comme l'a démontré Westinghouse (cf. Fig. 2) avec de tels plasmas les rendements de chauffage ainsi que la réduction du temps de chauffage peuvent être excellents. Notons également la possibilité, en utilisant des chambres de chauffage-réaction du type de celles mises au point par S.K.F. pour son procédé « Plasma-red » $[133,134]$, d'avoir une recirculation de l'air chauffé par plasma, seule une petite fraction de l'écoulement passant au travers des torches à plasma pour réchauffer la majeure partie de l'écoulement à des températures pouvant atteindre $2500^{\circ} \mathrm{C}$.

L'utilisation de la vapeur d'eau comme gaz plasma semble quant à elle très prometteuse [228] car :

- c'est un gaz peu coûteux,

- son enthalpie est considérable $\left(7,36 \times 10^{4} \mathrm{~kJ} / \mathrm{kg}\right.$ à $6000 \mathrm{~K}$, soit 25 fois l'enthalpie à $100^{\circ} \mathrm{C}$ à la pression atmosphérique),

- sa réactivité chimique une fois dissociée est importante,

- la séparation gaz-solide est simple par condensation,

- les applications peuvent se développer à pression élevée.

Si les générateurs du type Westinghouse ont déjà fonctionné avec de la vapeur d'eau, la seule application connue où le générateur fonctionne directement avec de l'eau est celle mentionnée par Alu-Suisse [89] avec un générateur de $500 \mathrm{~kW}$ utilisé pour la projection ou la vaporisation (cf. Fig. 9).

Des applications de ce type de générateur semblent se dessiner dans deux domaines très prometteurs :

- le traitement des hydrocarbures lourds ("char" et «tar ») par vapo-craquage pour lequel des compagnies pétrolières en Californie (U.S.A.) et en Alberta 
(Canada) ont débuté des recherches de faisabilité dont les premiers résultats semblent d'autant plus prometteurs qu'il s'agit là, semble-t-il, de la seule possibilité de transformer de tels produits qui sont en même temps désulfurés. Les études développées dans des plasmas RF d'argon [235, 236] pour le traitement des hydrocarbures ont démontré la pyrolise des huiles lourdes avec cependant des dépôts de suie préjudiciables au fonctionnement et qu'il convient d'éliminer en utilisant par exemple des plasmas d'hydrogène. Par contre les premières expériences développées à l'Université Mc Gill de Montréal [228] avec des huiles lourdes (5300 centistokes de viscosité à $40^{\circ} \mathrm{C}$ ) traitées dans un plasma de vapeur d'eau ont montré que $39,2 \%$ de l'huile était gazéifiée et que, en phase liquide, on retrouvait des huiles lourdes et des huiles légères;

- les sécheurs par atomisation fonctionnant avec de la vapeur "surchauffée » par plasma, c'est-à-dire avec des plasmas d'eau. Une telle substitution du plasma au chauffage par paroi permettrait [228, 237 et 238], en utilisant un générateur à plasma de $2,5 \mathrm{MW}$ à $6000 \mathrm{~K}$ opérant à $10 \mathrm{~atm}$, avec un débit d'eau de $0,027 \mathrm{~kg} / \mathrm{s}$ dans une chambre de $0,65 \mathrm{~m}$ de diamètre sur 4,84 m de hauteur, de remplacer un sécheur classique de même capacité de 5 à $6 \mathrm{~m}$ de diamètre et de 7,5 à $9 \mathrm{~m}$ de hauteur (dimensions fonction des conditions d'opération). Le coût d'investissement serait alors réduit de $43 \%$ et le coût de fonctionnement d'environ $50 \%$ [228]. Notons également que des générateurs à plasma à eau pourraient remplacer avantageusement les brûleurs des dispositifs d'évaporation par brûleurs immergés.

4.2.2 La production de gaz réducteur. - L'idée de base développée par S.K.F. en Suède [133, 134, 239], la société Cokerill en Belgique et Westinghouse [240, 231, 232] aux U.S.A., a consisté à adapter des dispositifs plasmas sur des systèmes classiques pour la production d'éponges de fer ou de fonte. Cette application conjugue l'action des gaz à haute température avec la réduction, ce qui conduit aux notions de : recirculation des gaz, régénération des propriétés chimiques, diminution du volume gazeux nécessaire, diminution de la teneur en réducteur nécessaire, amélioration du rendement énergétique. Le dispositif utilisé consiste à réformer un mélange gazeux partiellement oxydé $\left(\mathrm{CO}, \mathrm{CO}_{2}\right.$, $\mathrm{H}_{2}, \mathrm{H}_{2} \mathrm{O}$ ) par utilisation d'un vecteur électrique plasma et d'un combustible fossile (charbon ou fuel). Dans le procédé "Plasma-red» de S.K.F., maintenant à l'échelle industrielle (70 $000 \mathrm{~T} / \mathrm{an})$ pour la production d'éponge de fer destinée aux aciers spéciaux de roulement à bille, une partie des gaz extraits d'une tour classique de réduction d'éponge de fer (ancien procédé Wiberg-Soderfors) est chauffée et régénérée par apport de combustible fossile en utilisant des générateurs à plasma (Fig. 24). Il faut souligner que l'utilisation de 6 unités plasma de $1330 \mathrm{~kW}$ chacune alimentées avec $350 \mathrm{Nm}^{3} / \mathrm{h} /$ unité de gaz issus de la tour de réduction et ayant un rendement thermique de $85 \%$ a permis de remplacer l'ancien reformeur à coke chauffé par

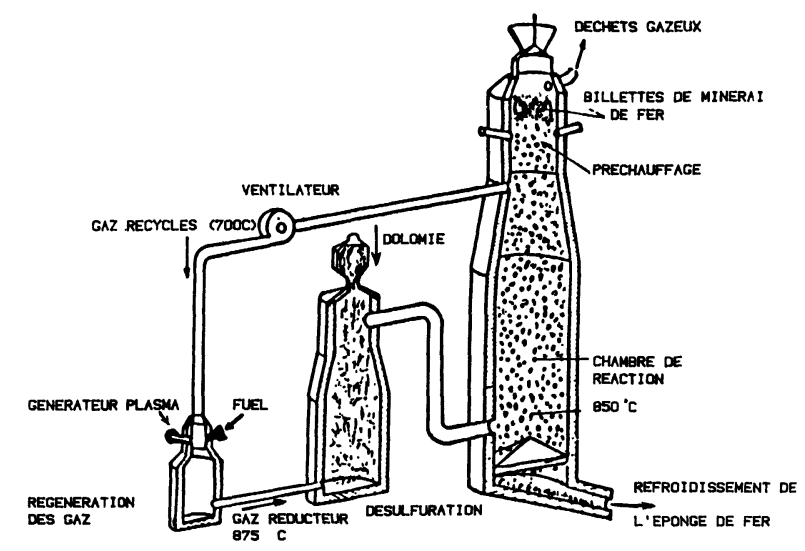

Fig. 24. - Procédé plasmared de S.K.F. pour la production d'éponge de fer à partir des gaz de sortie de la tour de réduction, gaz reformés avec du charbon ou du fuel).

[Plasmared process of S.K.F. for the production of sponge iron using reducing gases obtained from a plasma furnace (exhaust gases from the reduction tower reformed with coal fuel).]

résistance et permettant seulement une production de $25000 \mathrm{~T} / \mathrm{an}$. Le remplacement des électrodes toutes les 200 heures environ est parfaitement compatible avec l'utilisation industrielle normale du procédé. Le système de production de gaz réducteur (cf. Fig. 24) a d'abord été alimenté avec du gaz de pétrole liquéfié et il est maintenant opérationnel avec du charbon pulvérisé.

Soulignons que l'adaptation aux hauts fourneaux de ces dispositifs plasmas de production de gaz réducteurs à partir du charbon, peut permettre aussi de pallier au manque de coke dans l'industrie métallurgique (manque estimé à 4 MT en 1979 et prévu de 12 MT en 1985), puisqu'on éconotmise plus de $60 \%$ de la consommation en coke du dispositif classique [240, 241]. L'adaptation d'une telle solution aux hauts fourneaux a été démontrée par la société Cokerill à Seraing sur un haut fourneau de $500 \mathrm{~T} /$ jour. Cette solution utilise un générateur dont la puissance peut varier de 1500 à $3500 \mathrm{~kW}$ alimenté avec du gaz naturel sous des débits de 600 à $800 \mathrm{Nm}^{3} / \mathrm{h}$ et installé sur la tuyère du haut fourneau. Les débits de gaz et la puissance sont calculés pour fournir un rapport air/gaz en volume variant de 2,4 à 3,2 et entrant dans le haut fourneau à une température variant de 2000 à $2400^{\circ} \mathrm{C}$. Les premiers travaux indiquent une réduction de la consommation de coke à $180 \mathrm{~kg} /$ tonne de métal chaud contre 400 à $600 \mathrm{~kg} /$ tonne avec les HF classiques. L'avantage du procédé plasma dans ce cas comme dans le précédent est de s'adapter directement sur un procédé existant.

Les hauts fourneaux ont été la principale source de production du fer jusqu'alors, les plus modernes sont extrêmement sophistiqués et tendent à des productions très élevées de l'ordre de 2 millions de tonnes de métal chaud/an. Cependant il existe un besoin de procédés 
à des échelles plus réduites de 250000 à 500000 tonnes/an, soit pour les productions d'aciers spéciaux, soit pour les pays en voie de développement et c'est là que peut intervenir le procédé S.K.F. Plasmasmelt $[133,134,239]$. Dans la chambre d'un haut fourneau rempli de coke sont introduits le gaz plasma réducteur et les particules d'oxyde préréduites à réduire (cf. $\S$ II.3.3). Le coke sert alors de surface de réaction et de milieu filtrant à haute température pour la récupération de fer liquide. Le coke est alors consommé (50 kg/tonne) uniquement pour la carburation du métal liquide. Les gaz de sortie sont utilisés d'une part pour la préréduction en lit fluidisé et une partie, dépoussiérée, recompressée est utilisée comme gaz plasma dont le pouvoir réducteur est régénéré avec du charbon. Par rapport à un four traditionnel de $2 \mathrm{MT} /$ an le coût de production d'une tonne de fer plasma est évalué à 34,6 US (base 82) de moins.

- Une autre application potentielle mais certainement, à terme de 5 à 10 ans, est la gazéification des charbons pulvérulents avec des plasmas d'eau, traitement qui permet également leur désulfurisation et dont la potentialité a été mise en évidence par de récentes expériences à l'échelle de $80 \mathrm{~kW}$ [114]. Dans le même état d'esprit, des expériences de gazéification de la tourbe $[228,247]$ ont été développées à l'Université Mc Gill de Montréal en utilisant la technique de la suspension atomisée dans un réacteur chauffé par plasma d'eau. On obtient ainsi des gaz contenant 30 à $35 \%$ d'hydrogène et 35 à $40 \%$ de CO dont la conversion en méthanol est envisagée.

- Enfin, toujours dans ce cadre, il n'est pas impensable d'envisager l'utilisation de charbons pulvérulents dans des brûleurs assistés par plasma pour lesquels des expériences préliminaires ont montré que l'assistance plasma ne représenterait que $100 \mathrm{~kW}$ pour un brûleur de $60 \mathrm{MW}$ (à comparer au $2 \mathrm{MW}$ du brûleur d'assistance à fuel).

4.2.3 Les réactions chimiques. - C'est certainement là un des problèmes les plus complexes de l'utilisation des plasmas mais aussi un des plus passionnants. La complexité tient essentiellement à la rapidité des réactions, aux gradients considérables, à la difficulté d'introduire les réactifs, à la nécessité d'effectuer les opérations de trempe suivant des lois bien définies en fonction du temps, en les commençant ni trop tôt, ni trop tard. Les progrès de la métrologie et de la modélisation en ce domaine sont encore trop récents [146, $149,243,244]$ pour permettre une maitrise de ces phénomènes avant 5 ou 10 ans. Cependant, en contrepartie, ces réactions hors équilibre offrent des possibilités de rendement très intéressant (cf. § III.2.1). De plus, de récents travaux [182 à 185,189$]$ sur la catalyse hétérogène en plasma basse pression ont permis de montrer qu'il était possible, dans un plasma H.F., avec la même consommation énergétique de doubler certains taux de production comme celui des oxydes d'azote en présence d'une grille de $\mathrm{WO}_{3}$ ou $\mathrm{MoO}_{3}$ ou celui de $\mathrm{HCN}$ avec une grille de molybdène. Ces phénomènes encore très mal connus sont cependant extrêmement prometteurs surtout s'ils peuvent être étendus à la pression atmosphérique comme les premiers travaux le laissent entrevoir [189].

On comprend donc facilement pourquoi l'utilisation industrielle des réacteurs chimiques en plasma est encore limitée à quelques productions.

a) Les synthèses de petites molécules. - L'acétylène est un excellent exemple du passage, puis de l'abandon, puis peut-être à nouveau du passage d'un procédé plasma au stade industriel, avec les grands fours à plasma à l'échelle de $10 \mathrm{MW}$ développés par Hüls [92, 93], Hoechst, Dupont de Nemours [245] vers les années 1965 pour la production de l'acétylène à partir des hydrocarbures. De ces fours, abandonnés vers les années 1970, avec le développement de la voie éthylène meilleur marché, il ne reste actuellement en service que ceux de Hüls, encore qu'il soit envisagé par A.V.C.O. à la suite des premiers résultats obtenus $[108,109]$, de redévelopper le procédé mais cette fois-ci à partir du charbon. On utilise alors un arc à courant continu de $1 \mathrm{MW}$ dont la rotation rapide est assurée par un champ magnétique. L'acétylène formé dans l'arc doit être rapidement et efficacement trempé et le propane a été utilisé pour cette opération. L'énergie nécessaire est de l'ordre de $6,1 \mathrm{kWh} / \mathrm{kg}$ d'acétylène et d'éthylène produits. Il en va de même pour les travaux du Hüls [93, 246] qui a testé trois types de réacteurs avec une puissance de $400 \mathrm{~kW}$ chacun. En fonction de la granulométrie des poudres, du lieu d'injection, de la longueur du réacteur, du dispositif de trempe, un taux de conversion de $25 \%$ a été obtenu pour l'acétylène avec une consommation énergétique de $14 \mathrm{kWh} / \mathrm{kg}$. Les gaz sortant à $1800 \mathrm{~K}$ peuvent permettre la formation d'éthylène ou de propylène par trempe avec des hydrocarbures et enfin ils peuvent, étant encore à 500-1 $000 \mathrm{~K}$, chauffer de la vapeur. Pour le passage à $8 \mathrm{MW}$ par unité, le réacteur utilisant un vortex semble préférable car il fonctionne à une tension deux fois plus élevée que les autres configurations (1 $600 \mathrm{~V}$ contre 800). Des dispositifs similaires à ceux utilisés pour la production de l'acétylène peuvent également permettre la fabrication de l'acide cyanhydrique ou du noir de carbone comme cela a été démontré en laboratoire [247] ou par A.V.C.O. [108, 109].

L'étude de la production des oxydes d'azote par plasmas en est encore au stade du laboratoire. Mais, comme nous l'avons déjà indiqué, les expériences indiquent des taux de conversion supérieurs à ceux de l'équilibre $(12 \%$ contre $6,5 \%)[169,170]$ et les effets catalytiques semblent pouvoir améliorer ce taux de conversion $[184,185]$. Cependant, des études économiques détaillées sont encore nécessaires avant de passer à un prototype industriel.

b) Fabrication des réfractaires ou des pigments. Avec le développement des générateurs à plasma d'arc, a été démontrée [248] la possibilité de produire des poudres ultra-fines ayant une surface spécifique très 
élevée et des propriétés particulières. De telles poudres sont obtenues soit par vaporisation du solide correspondant suivie d'une trempe rapide des produits en phase gazeuse (comme par exemple la vaporisation au four à plasma de la silice en atmosphère réductrice produisant un gaz de $\mathrm{SiO}$ trempé à l'air [249, 250]), soit par réaction de l'halogénure en plasma d'hydrogène (comme la production du pigment de titane $\left(\mathrm{TiO}_{2}\right)$ à partir de $\mathrm{TiCl}_{4}$ en plasma d'oxygène [61]).

L'industrialisation de ces procédés a été réalisée pour les applications suivantes :

- réalisation de pigments de titane à partir de la réaction du chlorure de titane avec un plasma d'oxygène soit à haute fréquence [61] (à l'échelle de $400 \mathrm{~kW}$ en U.R.S.S.), soit à arc [251] par préchauffage de l'oxygène pour l'oxydation de $\mathrm{TiCl}_{4}$ par la tioxide à l'échelle de $1 \mathrm{MW}$;

- réalisation des poudres de silice ultrafines [249] obtenues à partir de la silice vaporisée en plasma avec des consommations énergétiques de l'ordre de 10 à $20 \mathrm{kWh} / \mathrm{kg}$ dans des fours à arc à l'échelle de 200 à $700 \mathrm{~kW}$. Les propriétés de ces poudres (submicroniques et sphériques) sous forme de chaînes avec des radicaux $\mathrm{OH}$, dépendent en fait de l'atmosphère et de lá vítesse de trempe au cours de la phase de condensationnucléation dont le mécanisme est très mal connu [252].

$\mathrm{Si}$ des métaux réfractaires (poudres de tungstène) ont été obtenus en plasma à des échelles pilotes, en laboratoire ont été étudiés un grand nombre de composés métalliques (nitrures, carbures, oxydes) avec souvent des phases cristallines particulières $(\beta$-WC, $\alpha \mathrm{Si}_{3} \mathrm{~N}_{4}$ ) [253 à 258], de telles poudres sont généralement submicroniques et mis à part peut-être $\mathrm{SiC}$ et $\mathrm{TiB}_{2}$, elles n'avaient pas jusqu'à présent d'utilisations technologiques à une échelle industrielle. Cependant, depuis un an, il semble bien qu'il y ait une recrudescence des travaux dans ce domaine avec des perspectives d'application.

Citons enfin la production de silicium par réduction de $\mathrm{SiCl}_{4}$ avec du sodium dans une installation de quelque $3 \mathrm{MW}$ chez Westinghouse [259, 260]. Toutefois ce programme n'a pas permis d'arriver à la qualité silicium solaire initialement recherchée.

\subsection{LES TRAITEMENTS EN PHASE HÉTÉROGÈNE.}

4.3.1 L'importance du domaine et les contraintes rencontrées. - Tous les pays dépendent plus ou moins des sources extérieures d'approvisionnement en matériaux bruts et doivent importer ceux-ci. Depuis quelques années, la possibilité d'une soudaine rupture d'approvisionnement est un scénario plausible : par exemple, les plus grandes réserves de nickel se trouvent à $\mathrm{Cuba}$, celles de chrome (près de $90 \%$ ) en U.R.S.S. et en Afrique Centrale et du Sud... De plus, la production d'un grand nombre de métaux est coûteuse en énergie généralement d'origine électrique. Il y a donc là une possibilité de développement des techniques plasmas, soit parce qu'elles conduisent à des rende- ments de conversion équivalents ou meilleurs, mais avec une souplesse infiniment plus grande que les techniques classiques de four à arc, soit parce qu'elles permettent le recyclage de nombreux métaux avec des caractéristiques de purification équivalentes à celles des traitements sous vide, mais avec un coût énergétique moindre. Ceci comprend le traitement :

- des déchets de fabrication,

- la récupération de déchets de mine ou de fines qui jusqu'alors n'étaient pas traités,

- la réalisation de nouveaux alliages avec substitution (par exemple, pour réduire l'utilisation du cobalt et du chrome),

- la diminution de certaines impuretés qui résultent actuellement d'une utilisation accrue de matériaux recyclés,

- la réalisation d'objets ayant une forme voisine de la forme finale, ce qui réduit les parties d'usinage.

Compte tenu de l'expérience sur les réacteurs plasmas, développés depuis maintenant près de dix ans, afin de remplir les différentes missions que nous venons d'énumérer, on peut affirmer d'un point de vue industriel qu'un dispositif plasma doit être capable de répondre aux contraintes techniques et à la viabilité économique suivantes [228] :

a) le système doit avoir un excellent rendement énergétique, ce qui est d'ailleurs probablement la contrainte la plus sévère du fait du coût élevé de l'énergie électrique,

b) les spécifications de pureté du produit doivent être tenues : dans certains cas, comme pour le molybdène, le taux de réaction doit dépasser 99,5\% [121],

c) la forme physique du produit final doit être acceptable, par exemple, dans de nombreux cas, on doit préférer un produit fondu à un produit pulvérulent submicromique,

d) les coûts d'investissement et d'opération doivent être raisonnables. Généralement, d'ailleurs, ils résident moins dans le générateur à plasma lui-même que dans son environnement, notamment pour l'alimentation en fluide et en poudres et dans l'automatisation du contrôle des paramètres,

e) les conditions de travail et d'environnement doivent respecter les standards classiques, ce qui est d'ailleurs souvent un des points forts des dispositifs plasma où le recyclage des effluents est souvent facile.

Pour illustrer notre propos, nous allons maintenant présenter quelques applications déjà industrialisées ou en passe de le devenir.

4.3.2 Fusion et purification en plasma. - La fusion de déchets métalliques a un potentiel de développement important avec l'enchérissement du prix des matières premières et les aléas de leur approvisionnement. De plus, avec le plasma, le raffinage du métal produit s'effectue simultanément avec la fusion. Les pertes par évaporation de composants intéressants comme le manganèse et le magnésium peuvent être très réduites, 
l'opération s'effectuant au voisinage de la pression atmosphérique. Enfin, l'atmosphère contrôlée permet le traitement d'alliages contenant des espèces facilement oxydables et chimiquement actives à haute température. Outre le fait que la puissance électrique, contrairement aux fours à électrodes, est indépendante de l'état d'avancement de l'opération et qu'il y a très peu de fluctuations, l'utilisation des espèces actives du plasma permet diverses transformations chimiques que l'on ne pourrait obtenir autrement [61] comme la surnitruration $(1,8 \%)$ dans la masse des aciers permettant peut-être de remplacer des aciers au nickel.

Pour les traitements des matériaux à forte valeur ajoutée, des fours couplant le chauffage inductif au chauffage plasma sont en service au Japon [261] depuis 1971, ainsi que des fours de refusion du titane (0,5 MW) au plasma chez Daido [262]. Leur installation comporte un creuset de $440 \mathrm{~mm}$ de diamètre avec 3 torches à plasma permettant d'obtenir une zone de chauffe assez large (puissance $540 \mathrm{~kW}$ ). La rotation du creuset ainsi qu'un champ magnétique entraînant la rotation du bain en fusion à la surface du lingot assure une excellente homogénéité chimique et thermique. Le dispositif appelé PPC présente de nombreux avantages : lable,

- plasma d'argon très propre et facilement contrô-

- fusion à la pression atmosphérique,

- traitement de matériaux variés : éponge de titane, déchets de titane, alimentés en continu au centre des trois jets de plasma.

On obtient ainsi en continu (le lingot au fur et à mesure de sa formation étant tiré vers le bas) des lingots de titane ou d'alliages de $3000 \mathrm{~mm}$ de long avec des consommations énergétiques de 1,6 à $2 \mathrm{kWh} / \mathrm{kg}$ et des densités apparentes de $3,2 \mathrm{~g} / \mathrm{cm}^{3}$ qui de plus satisfont aux normes de puretés JIS ou AMS. La Société belge Electrotherm [263] commercialise des fours à plasmas destinés à l'affinage du tantale, niobium, molybdène, à la fusion des alliages réfractaires au cobalt ou au nickel et des alliages de cuivre (puissance $250 \mathrm{~kW}$, consommation $5 \mathrm{kWh} / \mathrm{kg}$, production en lingots). Des études de faisabilité à grande échelle, notamment sur la qualité et l'homogénéité des lingots obtenus par refusion plasma pour des matériaux tels que le nickel, le cobalt... sont activement menées aux U.S.A. au centre de recherche de United Technologies à Hartford dans le Connecticut [264], refusion en arc continu transféré directement sur la surface du lingot. Le retraitement, par ce procédé, des déchets de titane ou d'alliages du type M.CrAlY (avec $\mathrm{M}=\mathrm{Ni}$, Co ou $\mathrm{NiCo}$ ) permet de retrouver un produit (sous forme de lingot) ayant toutes les caractéristiques requises par les normes, ce qui est très prometteur quand on sait que 50 à $70 \%$ en poids de ces métaux ou alliages à forte valeur ajoutée sont perdus dans les opérations de dépôt sous vide ou assistés par plasma ou de projection plasma.

Pour l'acier produit à partir des déchets métalliques, depuis le premier four à sole fonctionnant avec des torches plasmas [265], des unités industrielles susceptibles de traiter entre 10 et 30 tonnes en une seule opération ont été développées en U.R.S.S. et R.D.A. $[123,124]$, fours qui sont d'ailleurs commercialisés dans les pays occidentaux par la société autrichienne Voest Alpine [125]. La consommation énergétique varie de $3 \mathrm{kWh} / \mathrm{kg}$ au début de l'opération à $0,7 \mathrm{kWh} / \mathrm{kg}$ à la fin. Le gaz plasmagène est généralement l'argon (dont le débit est quasi indépendant de la puissance) et les durées de vie moyennes des électrodes sont de 300 à $400 \mathrm{~h}$, l'arc étant transféré entre 3 ou 4 torches et le bain en fusion. Les avantages principaux de ces fours plasma par rapport aux fours à arc classiques sont les suivants [125] :

- récupération maximale des alliages à partir des déchets

- réduction des pertes en fer,

- élimination d'une carburation indésirable durant la fusion,

- production d'aciers alliés de qualité comparable à celle des aciers au four électrique,

- élimination du bruit et amélioration des conditions de travail,

- élimination des transmissions d'énergie intermittentes.

Dans le même type d'opérations, on peut citer le réacteur de $1 \mathrm{MW}$ de Tetronics-Foster Wheeler [122] utilisé pour la fusion et la réduction : des fines de chromite, des laitiers, des matériaux contenant du zinc, du plomb et de l'argent. Il faut souligner à ce propos que les technologies plasmas sont les seules susceptibles actuellement de traiter les fines de minerais. Leur générateur a d'ailleurs été vendu à General Motors pour la fusion des copeaux d'acier et à Middelburg en Afrique du Sud à l'échelle de $8 \mathrm{MW}$ pour la production de ferrochrome.

La fusion-réduction d'un oxyde de fer préréduit à $65 \%$ pour produire une fonte à $3,5 \%$ de carbone est également développée à une échelle pilote de $10 \mathrm{MW}$ [266].

4.3.3 Vaporisation en plasma. - Ce travail a été développé à l'Université de Limoges [267] dans un four à plasma à arc transféré et au centre de recherche de Westinghouse [268] pour obtenir un aérosol de vapeur métallique destiné à la simulation d'un accident majeur dans une centrale nucléaire (Marviken Aerosol Transport Test Project). Il faut également noter que c'est là le biais de la production de poudres submicroniques d'acier dont l'usage, par exemple pour les aimants liquides, est susceptible de grands développements. Que ce soit les essais de Limoges jusqu'à $300 \mathrm{~kW}$ avec un arc transféré à l'hydrogène où les poudres d'acier sont injectées au voisinage de la pointe de cathode par effet Maecker ou ceux de Westinghouse avec une torche de $1 \mathrm{MW}$ alimentée en azote et où les poudres sont injectées en sortie du générateur qui est suivi d'un tube de carbone de $190 \mathrm{~mm}$ de diamètre 
interne et de $1500 \mathrm{~m}$ de long, tube isolé à la laine de carbone, des densités d'aérosol de $700 \mathrm{~g} / \mathrm{m}^{3}$ ont été atteintes avec des consommations énergétiques de 12 à $20 \mathrm{kWh} / \mathrm{kg}$ démontrant aussi la faisabilité d'une excellente vaporisation en plasma.

\subsubsection{Métallurgie extractive. - Nous ne parlerons} pas de l'adaptation des torches à plasma sur les hauts fourneaux (cf. § 4.2.2) qui semble être une application très prometteuse, et nous nous contenterons de décrire les opérations de métallurgie directe en plasma, opérations encore potentielles mais dont on peut estimer l'industrialisation à terme de 2 à 10 ans. En effet, l'utilisation des plasmas pour la production des métaux à forte valeur ajoutée comme les ferro-alliages ou le molybdène est étudiée depuis près de dix ans. Les travaux les plus récents dans ce domaine [228, 122, 123, $126,120,269$ ] semblent confirmer que la meilleure configuration possible est celle d'un arc transféré sur le bain en fusion servant d'anode. Les particules sont injectées au voisinage de la cathode pour arriver fondues sur le bain et le rayonnement vers les parois (ce type de plasma correspond à des arcs de 10 à $50 \mathrm{~cm}$ de long) est limité par l'injection d'une partie des matériaux pulvérulents en pluie autour de la colonne d'arc. Les études, menées à des puissances comprises entre 100 et $1000 \mathrm{~kW}$ ont essentiellement porté sur :

- le ferrovanadium [228, 270] pour lequel des consommations énergétiques de $5,5 \mathrm{kWh} / \mathrm{kg}$ ont été obtenues pour des produits contenant $5 \%$ de carbone ou de $12 \mathrm{kWh} / \mathrm{kg}$ avec moins de $0,6 \%$ de carbone, les deux produits contenant de 76 à $79,5 \%$ de vanadium,

- le ferrochrome $[271,272,122,268]$ avec une consommation énergétique comprise entre 6 et $12 \mathrm{kWh} / \mathrm{kg}$,

- le ferroniobium à partir du pyrochlore [228] le produit obtenu contenant $43 \% \mathrm{Nb}, 46 \% \mathrm{Fe}$ et $2,6 \% \mathrm{C}$,

- le molybdène $[228,121]$ avec un produit contenant moins de $0,085 \%$ de soufre et dont beaucoup d'impuretés $(\mathrm{Pb}, \mathrm{Bi}, \mathrm{Co}, \mathrm{P}, \mathrm{Mg}, \mathrm{Na}, \mathrm{K} . .$.$) sont prati-$ quement totalement éliminées. Signalons à ce propos que Noranda a cédé une licence de son four à la Société Davy Mc Kee (UK) qui le développe pour la métallurgie extractive des ferroalliages.

D'autres études sont également prometteuses au plan industriel mais cependant, à une échéance de 5 à 15 ans, comme celles de la valorisation de certains minerais par évaporation, comme la décomposition des silicates et de béryl [ 273 à 275], la récupération d'étain dans les laitiers [276, 277], la récupération de plomb [278] dans les bains à base de silicate, la récupération du fer à partir de la bauxite [279], la récupération de zinc et de plomb dans les fines des fours à arc avec le procédé plasmasdust de S.K.F. [280].

4.3.5 Autres applications. - Le traitement en plasma de tout matériau susceptible de fondre sans se vaporiser ou se décomposer permet d'obtenir, sous l'action des forces de tension superficielles, des particules sphériques. Ceci est utilisé industriellement pour produire :

- des sphères de zircone (1 000 t/an) [281], à partir de particules de zircon, sphères utilisées pour le frittage de céramiques ou la fabrication de certains verres ou de zirconium,

- des sphères de magnétite chez Westinghouse [282] et Alu Suisse [283] ou de ferrosilicium chez Rank Xerox [284] pour la photocopie.

Au plan de la recherche prédéveloppement, la faisabilité de la production de chamotte sphérique a été démontrée $[285,286]$ en utilisant un arc triphasé de $100 \mathrm{~kW}$ avec des électrodes en cuivre avec injection du gaz plasma (air) au voisinage des électrodes et l'étude du traitement des phosphates apatitiques par plasma [287] est intensivement menée à Minsk en U.R.S.S., d'autant plus que ce traitement semble conférer aux phosphates produits des propriétés de dissociation contrôlée dans le temps avec la formation d'une couche vitreuse, faiblement poreuse en surface.

Signalons également la possibilité de détruire divers déchets organiques comme la dioxine, l'arochlore, l'ascarèle... avec un four à plasma de $1 \mathrm{MW}$ à l'air où l'arc éclate entre une cathode tubulaire en cuivre et une anode en cuivre. La température dans le four n'est jamais inférieure à $1500 \mathrm{~K}$ et le rayonnement avec un arc de près de $1 \mathrm{~m}$ de long atteint $50 \%$ de la puissance dissipée. Les produits à traiter, sous forme liquide, sont introduits au voisinage de la cathode et le rendement de destruction atteint $99,99974 \%$ avec un coût énergétique variant de 0,54 à $2,4 \mathrm{kWh} / \mathrm{kg}$. Cette installation développée par la Compagnie Plasma Research de Kingston au Canada semble très intéressante.

4.3.6 Projection plasma. - La projection plasma [288 à 295] existe depuis vingt-cinq ans maintenant et son développement au cours de ces dernières années est quasi exponentiel puisque depuis 3 ans aux U.S.A. le chiffre d'affaires double presque tous les ans. Cet intérêt croissant pour cette technique de dépôt est dû au fait qu'avec la solidification rapide des particules projetées et des dépôts obtenus on combine en une seule opération la fusion, la trempe et la consolidation des matériaux, ce qui confère aux dépôts des propriétés uniques :

- très grande variété de matériaux déposés et versatilité des dispositifs de projection,

- dépôts dont les propriétés sont indépendantes des contraintes thermodynamiques de composition,

- possibilité de faire varier les concentrations de défauts dans de larges limites,

- possibilité, du fait des vitesses de trempe (jusqu'à $10^{6} \mathrm{~K} / \mathrm{s}$ ), d'obtenir des phases métastables ou des matériaux amorphes,

- génération de microstructures différentes de celles obtenues avec les procédés conventionnels, en particulier des tailles de grains submicroniques, 
— possibilité de fabriquer entièrement des pièces par projection y compris avec des matériaux très cassants.

La projection est effectuée avec des torches du type de celles décrites au $\S 2.2 .1 a$ ) et représentées sur la figure 6 et fonctionnant à des puissances comprises entre 20 et $100 \mathrm{~kW}$ avec des gaz (généralement $\mathrm{Ar}, \mathrm{N}_{2}$ ) contenant un pourcentage important (jusqu'à $50 \%$ ) d'hydrogène ou d'hélium afin d'améliorer le transfert thermique ( $c f . \S 3.3 .2$ ). Les poudres dont la granulométrie, suivant leur aptitude à fondre, varie entre 10 et $20 \mu \mathrm{m}, 20$ et $40 \mu \mathrm{m}, 30$ et $60 \mu \mathrm{m}$ en moyenne sont injectées dans ou à la sortie de la tuyère (au-delà du point d'accrochage de l'arc), soit perpendiculairement à l'axe du jet, soit avec une légère inclinaison à contre courant (la vitesse d'injection est un des paramètres les plus importants [291]). Les générateurs fonctionnant soit à la pression atmosphérique à l'air libre (projection d'oxydes essentiellement), soit, pour éviter l'oxydation, sous vide partiel (autour de 50 torr) dans une chambre refroidie pour la projection des métaux, des alliages, des carbures et des nitrures. Les principales applications [291, 290, 293 à 295] sont essentiellement pour des dépôts contre l'usure, la corrosion, l'oxydation, pour des barrières thermiques ou pour des dispositifs anti-frottement. Parmi les matériaux les plus utilisés, il faut citer les oxydes $\left(\mathrm{Al}_{2} \mathrm{O}_{3}, \mathrm{ZrO}_{2}, \mathrm{Cr}_{2} \mathrm{O}_{3}\right.$ notamment) et les alliages du type MCrAlY où $\mathrm{M}$ est soit $\mathrm{Ni}$, soit $\mathrm{Co}$, soit $\mathrm{NiCo}$ utilisés pour les aubes de turbine.

\section{Conclusion.}

Nous ne prétendons pas avoir présenté l'ensemble des applications actuelles et potentielles des plasmas thermiques de puissance, mais nous avons tenté d'en souligner les avantages et les inconvénients ainsi que les développements potentiels. Outre la projection plasma utilisée industriellement depuis plus de 20 ans et actuellement encore en plein développement, un nombre réduit d'applications est maintenant passé au stade industriel pour des applications spécifiques où la technique plasma est sans concurrence. Cependant, depuis deux ou trois ans, on voit se développer des applications comme la production de certains aciers où la technique plasma est venue non pas se substituer à l'ensemble d'un procédé, mais s'insérer dans le procédé lui-même. Ce développement industriel des techniques plasma est dû, d'une part, aux avantages propres du plasma :
- utilisation rationnelle de l'énergie,

- fiabilité et simplicité,

- mise en route et arrêts rapides,

- compatibilité avec les processus existants,

- traitement direct avec des matériaux bruts même sous des formes inutilisables dans les procédés classiques comme les fines,

- possibilité de produire par projection des dépôts épais (jusqu'à plusieurs $\mathrm{mm}$ ) céramiques ou métalliques ayant des propriétés très spécifiques avec parfois même des qualités supérieures à celles des pièces réalisées par des méthodes classiques,

et, d'autre part, à la crise énergétique qui fait que, pour la majorité des pays industrialisés, l'énergie électrique devient en termes de coût et de disponibilité une des énergies essentielles que les plasmas utilisent au mieux.

Ces développements industriels passent par l'étude, la mise au point et la réalisation de générateurs à plasma d'une puissance comprise entre 3 et $20 \mathrm{MW}$, de nouveaux types de réacteurs avec des températures et vitesses plus uniformes, de distributeurs de poudres adaptés à la technologie plasma (débits réguliers pouvant varier dans des limites importantes et en atmosphère contrôlée), de dispositifs de trempe.

Cependant, il ne faut pas oublier que ces développements sont aussi conditionnés par une meilleure compréhension des phénomènes, afin de contrôler et d'améliorer les rendements de conversion et les consommations énergétiques. En effet, beaucoup de recherches fondamentales ont été court-circuitées par l'espoir d'exploiter rapidement une technologie nouvelle et de nombreux développements prometteurs n'ont pu atteindre l'étape d'une technologie viable. Une recherche de base intensive est donc nécessaire en parallèle avec le développement industriel de certains processus pour compter le vide entre les phénomènes de base dans l'arc et/ou les phénomènes plasma et la physique de la chimie des processus plasmas. Ceci est particulièrement vrai pour les points suivants :

- les transferts de chaleur et de quantité de mouvement plasma-particules,

- l'introduction des solides et des gaz froids dans les plasmas thermiques,

- les écarts à l'équilibre, la cinétique des réactions vibrationnelles et la catalyse en plasma,

- la modélisation des écoulements et des cinétiques de réaction,

- les propriétés de transport des mélanges complexes, tels C-H-O-M... (où $\mathrm{M}$ est un métal). 


\section{Bibliographie}

[1] Von ENGEL, A., Electron plasmas : their nature and uses (ed.) International Publications Services (Taylor and Francis Inc. NY) 1983.

[2] PFender, E., Plasmas chemistry fundamental concepts, Course on fundamentals and applications of plasma chemistry (ed.) University of Minnesota Minneapolis USA (1979).

[3] Baronnet, J. M., Contribution à l'étude spectroscopique des plasmas d'azote produits par un générateur à arc soufflé ; application à la chimie des plasmas : synthèse des oxydes d'azote. Thèse d'Etat Université de Limoges, nov. 1978.

[4] Chen, D. M. and PFender, E., IEEE Trans. Plasma Sc. PS 9 (4) (1981) 265.

[5] Chen, D. M., Hsu, K. C., Pfender, E., Plasma Chem. Plasma Process. 1 (3) (1981) 295.

[6] Storey, S. H., VAN Zegeren, F., The computation of chemical equilibria (ed.) (Cambridge University Press) 1970.

[7] Brinckley, S. R., J. Chem. Phys. 34 (1947) 2.

[8] Huff, Gordon, Morrel, NACA, Report (1951).

[9] White, S. M., Johnson, S. M., Dantzig, G. R., J. Chem. 28 (1958) 5.

[10] Glouchko, V. L., Propriétés thermodynamiques des corps purs (ed.) Mir, Moscou (1962).

[11] JANAF, Thermodynamics Tables, US Dept. of Commerce, NBS, Washington (1971).

[12] ZelenicK, F. J., Gordon, S., Simultaneous least squares approximation of a function and its first integrals with applications to thermodynamic data, NASA-TN, p. 767

[13] Fauchats, P., Baronnet, J. M., Bayard, S., Rev. Int. Htes Temp. Réfract. 12 (1975) 221.

[14] Drellishak, K. S., Ph. D. Thesis, Northwestern Univ. (1963).

[15] Capitelli, M., Ficocelli, E., Molinari, M., Equilibrium compositions and Thermodynamic properties of mixed plasma (ed.) Instituto di Chimical General e Inorganica, Universita degli Studi, Bari, Italy (1969).

[16] HiRsChFeldeR, J. D., Molecular Theory of Gases and Liquid (John Wiley) 1954.

[17] Jeans, J. G., The Dynamical Theory of Gases, 4th Edition (ed.) (Cambridge University Press, Cambridge) 1925.

[18] Chapman, S. and Cowling, T. G., The Mathematical Theory of Non-Uniform Gases (ed.) (Cambridge University Press, Cambridge) 1958, 2nd edition 1970.

[19] Cambel, A. L. and Fenn, J. B., Transport Properties in Gases (ed.) (Northwestern University Press, Evanston III) 1958.

[20] Mason, E. A., Kinetic processes in gases and plasmas (ed.) A. R. Hochstim (Academic Press, N. Y. and London) 1969.

[21] Devoto, R. S., Phys. Fluids 10 (1967) 2105.

[22] Dalgarno, A., Philos. Trans R. Soc. London A250 (1958) 425.

[23] Athye, W. F., A critical evaluation of methods for calculating transport coefficients of partially and fully ionized gases, NASA TN, ND-2611 (1965).
[24] Devoto, R. S., Phys. Fluids 9 (1966), 1230

[25] Devoto, R. S., LI, C. P., J. Plasma Physics 2 (1968) 17.

[26] Devoto, D. S., A.I.A.A. J. 7 (1969) 2.

[27] Devoto, R. S., Phys. Fluids 16 (1973) 616.

[28] BonNefor, C., Thèse 3e cycle, Université de Limoges, 1975.

[29] Butler, J. N., Brokaw, R. S., J. Chem. Physics 26 (1957) 1636.

[30] Vanderslice, J. T., Weisman, S., Mason, E. A., Fallon, R. J., Phys. Fluids 5 (1962), 155.

[31] Emmons, H. W., Modern Developments in heat transfer (ed.) W. Ibelec (Academic Press New York) 1963.

[32] Report of the IUPAC sub-commission on plasma chemistry : Thermodynamic and transport properties of pure and mixed thermal plasmas at LTE, Pure Appl. Chem. 54 (1982) 1221.

[33] Aubreton, J., Fauchais, P., Influence des potentiels d'interactions sur le calcul des propriétés de transport, Revue Phys. Appl. 18 (1983) 51.

[34] FEY, G., Electric arc heater for the process industries, National Industrial Electric Heating Conference, Cincinnati, Ohio, USA feb. 9-12 (1976).

[35] Capitelli, M., Gorse, C., Fauchais, P., J. Chimie Physique 78 (1976), 655.

[36] Capitelli, M., Gorse, C., Fauchais, P., J. Physique 38 (1977) 653.

[37] Potapof, A. V., High Temperature 4 (1) (1966) 48.

[38] Daybelge, U., Transport properties of tw\%-temperature partially ionized plasmas. Ph. D. Thesis, Stanford University (1968).

[39] Donskoi, A. V., Goldfarb, V. M. and Flubnikin, V. S., Physics and technology of low-temperature plasmas (ed.) S. V. Dresvin (1972) English edition translated by $\mathrm{T}$. Cheron and edited by $\mathrm{H}$. U. Eckert. (Iowa State University Press) 1977.

[40] Miller, E. J. and SAndler, S. I., Phys. Fluids 16 (4) (1973) 497.

[41] Kannapan, D. and Bose, T. K., Physics Fluids 16 (5) (1973) 616

[42] Ksu, K. C. and PFender, E., Calculation of thermodynamic and transport properties of a low-temperature argon plasma 5 th International Symposium on Plasma Chemistry, Heriot Watt University, Edinburgh, 10-14 august (1981). Proceedings, p. 144.

[43] BoNNEFOI, C., Contribution au calcul des propriétés de transport d'un plasma à deux températures. Thèse d'Etat Université de Limoges, mars 1983.

[44] Mexmain, J. M., Coefficients de transport dans un plasma à deux températures, Application du mélange argon-oxygène. Thèse $3^{\mathrm{e}}$ cycle. Université de Limoges (31 oct. 1983).

[45] Bonnefoi, C., Aubreton, J., Mexmain, J. M., FauCHAIS, P., The transport properties of a two temperature $\mathrm{Ar}-\mathrm{H}_{2}$ plasma. Soumis à Plasma Proc. Plasma Chem.

[46] Devoto, R. S., The transport properties of a partially ionized monoatomic gas $\mathrm{Ph}$. D. Thesis, Stanford University (1967).

[47] Pfender, E., Arcs and plasmas torches in Plasma 
Technology and Application Continuing Education, 2nd World Congress of Chemical Engineering and World Chemical Exposition, Montreal, Canada (1981), oct. 4-9.

[48] Pfender, E., Electric Arcs and Arc Gas Heaters in Gaseous Electronics 1, p. 291, (ed.) M. J. Hirsch, H. J. Oskam (Academic Press N.Y.) (1978).

[49] Fauchais, P., Rev. Int. Hautes Temp. Réfract. 5 (1968) 71.

[50] Leontiev, A. J. et Voltshrov E. P., Caractéristiques électriques et thermiques d'un plasmatron de haute enthalpie in Investigations expérimentales des plasmatrons. M. F. Joukov Ed. Nauka Novossibirsk (1977)

[51] Joukov, M. F., Kourotchkine et Poustagarov, A. V., Hydrodynamique appliquée des plasmas thermiques. Rédacteur : Koutatieladze S. S. ed. Nauka Novossibirsk (1975).

[52] Joukov, M. F., Kourotchkine et Poustagarov, A. V., Etude des plasmatrons utilisant des gaz plasmagènes soufflés à travers un étage rapporté entre les électrodes, p. 82, in Investigation expérimentale des plasmatrons, M. F. Joukov Ed. : Nauka Novossibirsk (1977).

[53] Valbona, G., Bonet, C. et Foex, M., J. Phys. D. Appl. Phys. 8 (1975) 1185.

[54] Fey, M. G. et Kemeny, G. E. A., Method of direct ore reduction, a short gas arc heater, U.S. Patent 3, 765,870 , oct. 16 (1973).

[55] Shakov, M. F., Smolyakov. V. Ya. et Urgurov, B. A., Electric arc heaters of gases ed. : Nauka Moscow (1973).

[56] Schnell, C. R. et al. (Lonza L.T.D.), The Industrial application of plasma technology for the production of fumed silica, Symposium on Commercial potential for arc and plasma process, Atlanta City, N.J., 8-11 sept. (1974).

[57] Joukov, M. F., Calcul de base des générateurs à plasma, ed. : Nauka Novossibirsk (1979) (en russe).

[58] Fey, M. G., Electric Arc Plasma Heater for the Process Industries, Ind. Heat June (1976).

[59] Kassabji, F., Fauchais, P., Revue Phys. Appl. 16 (1981) 549.

[60] SAYCE, I. G., Plasma processes in extractive metallurgy, in Advances in Extractive Metallurgy and Refining, London oct. 4-6 (1977).

[61] Rykalin, N. N., Pure Appl. Chem. 48 (1976) 179 et $52(1980) 1801$.

[62] Hamblyn, S. M., Minerals Sci. Eng. 9 (1977) 151.

[63] Gold, D., Revue de la technologie des plasmas thermiques, Nouvelles applications de l'électricité, Colloque International, Toulouse 7-11 mars (1977), contribution VI-3 (ed. CFE Paris).

[64] Fauchais, P., Bourdin, E., Ceramics and Plasma, proceedings of the III International Symposium on Plasma Chemistry, Limoges 13-19 july (1977), invited paper. (ed.) P. Fauchais, Université de Limoges.

[65] Bonet, C., Pure Appl. Chem. 52 (1980) 1707.

[66] Aubreton, J., Fauchais, P., Rev. Gen. Therm. 200 (1978) 681.

[67] Fauchais, P., Bourdin, E., Coudert, J. F., Actualité Chimique 10 (1981) 15.

[68] Aubreton, J., Pateyron, B., Fauchais, P., Rev. Gen. Therm. 18 (1981) 293.

[69] FAUCHAIs, P., Les réacteurs et fours à plasma, J. Four
Electrique 7 (1982) 9, 9 (1982) 7, 10 (1982) 29, 11 (1983).

[70] Guile, A. E., Arc Electrode Phenomena Proc. I.E.E., I.E.E. Rev. 118 (1971) 1131-1154.

[71] GuILE, A. E., Erosion on non-refractory cathodes in arc plasma devices Proc. 4th Int. Symp. on Plasma Chem. Zurich (1979).

[72] Santen, S., Plasma smelting ISPC 6, Montreal, july 83, ed. Prof, Boulos University of Sherbrooke, Canada.

[73] George, A. P., Harel, A. L., Arc Heater operating characteristics in oxygen and nitrogen, an interim report, ISPC 6 Montreal july 83 ed. : Prof. Boulos University of Sherbrooke Canada.

[74] Fey, M. G., Melilli, W. J., The Application of thermal plasma systems to economical scale ironmaking ISPC 5, Edinburgh, aug. 1981 (ed.) B. Waldie, Univ. of Edinburgh, G. B.

[75] Kanzawa, A., Pfender, E., Numerical Analysis of the joule heating effect on plasma heat transfer, I.E.E.E. Trans. Plasma Sci. PS-6 (1978) 33-42.

[76] Johnson, D., Pfender, E., Modeling and Measurement of the initial anode heat fluxes in pulsed highcurrents arcs, I.E.E.E. Trans. Plasma Sci. PS-7 (1979) 44-48.

[77] SMITH, J. L., PFENDER, E., Determination of local anode heat fluxes in high intensity, thermal arcs, I.E.E.E. Trans. Power Appar. and Syst. PAS-95 (1976) 704-710.

[78] Dinulescu, H. A., Analysis of the anode boundary layer of a high pressure high current arc, $\mathrm{Ph} . \mathrm{D}$. Thesis, Univ. of Minnesota (1979).

[79] Heberlein, J. V. and PFender, D., Investigation of the anode boundary layer of an atmospheric pressure argon, I.E.E.E. Trans. Plasma Sci. PS-5 (1977) 171-180.

[80] Dinulescu, H. A. and Pfender, E., Analysis of the anode boundary layer of high intensity arcs, $J$. Appl. Phys. 51 (1980) 3149-3157.

[81] Pfender, E., Energy Transport in Thermal Plasmas, Pure Appl. Chem. 52 (1980) 1773-1800.

[82] Vardelle, A., Fauchais, P., Vardelle, M., Projection de revêtements protecteurs par plasma. Actualité Chimique 10 (1981) 69.

[83] Bourdin, E., Calcul simplifié de l'adaptation d'une tuyère de générateur à plasma de projection. Rapport Interne au Laboratoire de Thermodynamique 2 (1980) Université de Limoges.

[84] Joukov, M. F., Basical calculations of plasmatrons (en russe) ed. Nauka Novossibirsk (1979).

[85] Joukov, M. F., Production de plasma à basse température (en russe) Séminaire franco-soviétique à l'IVTAN, Moscou (1979) publié par l'académie des Sciences d'URSS.

[86] Hartnau, J., Acurex Corp. Mountain View, CA, USA, Acurex, arc plasma heater technology, Workshop on industrial Plasma Developments, Sherbrooke Quebec Canada, paper to be asked to the author.

[87] MikLossy, K., CSSR patent application $n^{0} 7083-63$ and 7084-63.

[88] Gross, B., Gricz, B. and Miklossy, F., Plasma Technology (Iliffe Bokks Ltd, London) 1968.

[89] Schnell, C. F., Hamblyn, S. M. L., Hengartner, K. and Wissler, M., Powder Technol. 20 (1978) 15-20. 
[90] Zhidovich, A. I., KravchenKo, S. K., Yasko, O. I., Plasma generator with bilateral outflow and electrodes with variable diameter, Inzh. Fiz. Zh. 15 (1968) 204.

[91] ShashKov, A. G., YaSko, O. I., Application of approximate similarity for correlarity arc characteristic, IEEE Trans. Plasma Sci. PS 1 (1973) 21-37.

[92] Gladish, H., Hydrocarbon Process. Pet. Refines. 41 (1962) 159.

Gladish, H., Chimie Ingenieur Technick 41 (1969) 204.

[93] MülLER, R., Recent developments for the production of acetylene from coal by the Hüls arc process, ISPC 6, Montréal, july 83 (ed.) Prof. Boulos, Univ. of Sherbrooke.

MülleR, R., Chemische Werke Hüls, Marl, West Germany The Hüls arc Process, Workshop on industrial plasma developments, Sherbrooke, july 21-23 (1983) paper to be asked to the author.

[94] Bonet, C. et al., J. Phys. D. Appl. Phys. 9 (1976) L 141.

[95] Shakow, M. F., Smolyakov, V. Ya., Urgukov, B. A., Electric-Arc Heaters (ed.) Nauka, Moscow (1973).

[96] Bonet, C., LAmos, J., FoeX, M., Ecoulements permanents de plasma de grande puissance, Entropie 34-35 (1970) 36-48.

[97] BONET, C., Les générateurs de plasmas à arc gainé, High Temperatures - High Pressures 3 (1971) 333-343.

[98] Gold, D., Bonet, C., Chauvin, G., Mathieu, A. C., Spheroidisation of aluminosilicate particles in a three-phase A.C. plasma furnace, Proc. of 4th Int. Symp. on Plasma Chemistry. Zurich, september (1979).

[99] Baronnet, J. M., Coudert, J. F., Rakowitz, J., Bourdin, E. et Fauchais, P., Nitrogen oxides synthesis in a DC plasma jet; 4th ISPC, Zurich august 27, sept. 1st (1979) ed. S. Veprek, University of Zurich.

[100] Coudert, J. F., Bourdin, E., Fauchais, P., Plasma Chem. Plasma Proc. 2 (1982) 399.

[101] Mc Kelliget, J., Szekely, S., Vardelle, M., FauChais, P., Plasma Chem. Plasma Proc. 2 (1982) 317.

[102] Lee, Y. C., Hsu, K. C., Pfender, E., Modelling of particles injected into a d.c. plasma jet, in the proceedings (p. 795) of the 5th ISPC, Edinburgh 10-14 aug. (1981) ed. B. Waldie Heriot Watt University.

[103] Chen, D. M., Hsu, K. C., Pfender, E., Plasma Chem. Plasma Proc. 3 (1981) 295.

[104] Vardelle, A., Vardelle, M., Fauchais, P., Plasma Chem. Plasma Proc. 2 (1982) 255.

[105] Pickles, C. A., W'ang, S. S., Mclean, A., Alcock, C. B. et Segworth, R. S., Transactions ISIJ 18 (1978) 369

[106] WhymaN, D., J. Scient. Instrum. 44 (1967) 525

[107] TyLKo, J. K., High Temperature Treatment of Materials, Can. Pat. no 957773 Granted to Tetronics Ltd. (1974).

[108] Arc-Coal Process Dev. Final Report May (1966), april (1972), AVCO Corporation System Division, Towell, Massachussets, U.S.A. 91851.

[109] Patrick, Jr A. J., AVCO Corp. Wilmington Mass,
USA, Workshop on Industrial Plasma Development Univ. of Sherbrooke, july 21-23 (1983). Paper to be asked to the author.

[110] TyLko, J. K., Reszhe, E., Studies in the SSP systems, modifications of the arc discharges at high orbital speeds, ISPC 6, july 83, Montreal (ed.) Prof. Boulosj Univ. of Sherbrocke Canada.

[111] MaeCKer, H., Z. Physik 135 (1953) 119.

[112] Wilks, P. H. et al., Chem. Eng. Proc. 68 (1972) 82.

[113] SheER, C., Korman, S. et Kang, S. F., Investigation of convective arcs for the simulation of re-entry aerodynamic heating. AFORSR-TR-74-1505, Contrat F-44 620-69-C-0104, 1974.

[114] Sheer, C., Korman, S., Dougherty, T. J., Arc gasification of coal, 4th International Symposium on Plasma Chemistry, Zurich Sept. (1979) Proceedings, p. 277.

[115] Bayliss, R. K., Bryant, J. W. et SAYCE, I. G., Plasma dissociation of zircon sands in the proceedings (S-5-2) of the III International Symposium on Plasmas Chemistry, Limoges France 12-19 juillet 1977.

[116] Tsantrizos, P., Gauvin, W. H., Can. J. Chem. Eng. 60 (1982) 822.

[117] Yerouchalmi, D. et al., High Temperatures, High Pressures 3 (1971) 271.

[118] HowIE, F. H. et SAYCE, I. G., Rev. Htes Temp. Réfract. 11 (1974) 169.

[119] Golf, R. G., Sandall, W. R., Chepilick, P. G. et MAC REA, D. R., Plasma reduction of iron oxide with hydrogen and natural gaz at $100 \mathrm{~kW}$ and $1 \mathrm{MW}$, International Round Table on Study and Application of Transport Phenomena in Thermal Plasmas. IUPAC Odeillo, 12-16 sept. 1975, ed. Labo Ultra Réfractaires.

[120] Pateyron, B., Aubreton, J., Kassabji, F., Fauchais, $P$., New design of reduction plasma furnace including electrical transfer to the bath and falling films, IVth International Symposium on Plasma Chemistry (ed.). B. Waldie Univ. Edinburgh (1981) p. 167.

[121] Gauvin, H. W., Kubanec, G. R., Irons, C. A., J. Metals 33 (1981) 42.

[122] Monk, J. R., Application of plasma to metallurgical processes. Vth International Symposium on Plasma Chemistry (Ed.) B. Waldie Edinburgh (1981) p. 162.

[123] Dembovsky, V., Possibilités du chauffage plasma pour la production de métal dans les fours à creusets horizontaux, 9e Congrès International de l'UIE Cannes 20-24 oct. (1980) (session $n^{\circ} 25$ ).

[124] Barodachyov, A. S. et al., Design of ceramic crucible plasma furnaces and operating results, $9 \mathrm{e}$ Congrès International d'Electrothermie, Session 81, 23/10/ 81, Cannes, ed. C.F.E. 79 rue de Miromesnil, Paris.

[125] SCHARF, G., Comparison of the plasma primary melting process with the conventional electric arc furnace, Proc. of the 7th ICVM 1982. Tokyo Japon.

[126] Carr, T., Barcza, N. A., Maske, K. U., Mooney, J. F., The design and operation of transferred-arc plasma systems for pyrometallurgical applications, ISPC 6 Montréal, july 83 (ed.) Prof. Boulos University of Sherbrooke CN.

[127] Harry, J. E., Knight, R., Power Supply design for 
multiple discharge arc processes. ISPC 6, Montréal, july 83 (ed.) Prof. Boulos, Univer. of Sherbrooke.

[128] FIEDLER, H. et al., Results of plasma melting of steel. Fifth International Symposium on Electroslag Remelting and Other Special Melting Process, Pittsburg 1974.

[129] SCHOUMAKER, H. R. P., Fours à plasma, in proceedings of International Round Table on Study and Application of Transport Phenomena in Thermal Plasmas, IUPAC Odeillo 12-16 sept. 1975. Ed. Laboratoire des Ultra Réfractaires.

[130] Kinoshito, T., Shinka 17 (1975) 61.

[131] Yamaguchi, K., Int. Indus. Seminar on Pilot Plant Experiences Melting and Processing Technology. Seabrook Island, South Carolina oct. 80. Possibilité de se procurer le rapport en écrivant à Daido Steel Co, R and D Division Nagoya, Japon.

[132] Roman, W. C., United Technologies Research Center, East Hartford, Conn, USA Plasma Processing for materials reclamation Workshop on Industrial Plasma Development, Univ. of Sherbrooke, july 21-23, paper to be asked to the author.

[133] Santen, S., Plasma technology gives new lease on life Swedish D. R. Plant Iron and Steel International (december 1979).

[134] AdESSON, J., Development of Plasma, Real process from pilot plant to production, Paper presented at International Seminar on Pilot Plant Experiences, Melting and Processing Technology, Seabrook Island South Carolina USA (sept. 1980) et H. Herlitz, "Plasmared and Plasmasmelt processes " Rapport interne S. K. F. Hofors, Suède mars (1980).

[135] Young, R. M., Chyon, Y. P., Fleck, E., Pfender, E., An experimental arc plasma reactor for the synthesis of refractory materials, ISPC 6, Montreal july 83 (ed.) Prof. Boulos, Univ. of Sherbrooke $\mathrm{CN}$.

[136] Hsu, K. C., Etemadi, K., Pfender, E., J. Appl. Phys. 54 (1983) 1293.

[137] Mazza, A., Pfender, E., Modelling of an arc plasma reactor for thermal plasma synthesis, ISPC 6 , Montreal july 83 (ed.) Prof. Boulos Univ. of Sherbrooke, CN.

[138] Patankar, S. V., Numerical heat transfer and fluid flow (New York, Mac Graw-Hill) 1980.

[139] LeOnard, P. B., Comput. Meth. Appl. Mech. Eng. 19 (1979) 59.

[140] Ushio, M., Szekely, J., Chang, C. W., Mathematical modelling of flow field and heat transfer of high current arc discharge, to be published, Department of Material Science and Engineering MIT, Cambridge, Ma 02139 .

[141] Jones, W. P., Whitelaw, J. W., Combustion Flame 48 (1982) 1.

[142] Correa, S. M., Translational Plasma Jet Modelling ISPC 6, Montreal july 83 (ed.) Prof. Boulos, Univ. de Sherbrooke CN.

[143] Stine, H. A., Watson, V. R., The theoretical enthalpy distribution of air in a steady flow along the axis of a d.c. electric arc NASA, TN, D-1331 (1962).

[144] Watson, V. R., Pegot, E. B., Numerical calculations for the characteristics of a gas flowing axially through a constricted arc, NASA, TN, D-4042 (1967).
[145] Liu, G. N., Chu, F. Y., Simpson, C. J., Design and laboratory utilization of a thermal plasma generator, ISPC 6, Montreal july 83 (ed.) Prof M. Boulos Univ. de Sherbrooke, CN.

[146] Vardelle, A., Baronnet, J. M., Vardelle, M., FAuchais, P., IEEE Trans. Plasma Sci. PS 8 (1981) 417.

[147] Etemadi, K., Pfender, E., Rev. Sci. Instrum. 53 (2) (1982) 255.

[148] Mehmetoglu, M. T., Kitzinger, F., Gauvin, W. H., Rev. Sci. Instrum. 53 (2) (1982) 285.

[149] Coudert, J. F., Baronnet, J. M., Fauchais, P., Diagnostic on a d.c. plasma jet using an $O M A$, ISPC 6, Montreal, july 83 (ed.) Prof. M. Boulos, Univ. de Sherbrooke CN.

[150] Lesinski, J., Misera-Lesinska, B., Jurewicz, J., Boulos, M., Particle and gas velocity measurements in a D.C. plasma jet, 88th A.I.Ch.E., Nat. Meeting, Philadelphia, Pen. USA, june 8-12 (1980).

[151] Barrière, M., Prud'homme, R., Equations fondamentales de l'aérothermochimie (Masson et Cie Editeurs Paris) 1973.

[152] Frank-KameneStKII, D., Diffusion and heat transfer in chemical kinetics (Plenum Press, New York, London) 1969.

[153] Emanuel, N., Knorre, D., Cinétique chimique (Edition Mir. Moscou) 1975.

[154] Johston, H., Gas phase reaction rate theory (Ronald Press Company New York) 1966.

[155] Gladstone, S., Laidler, J. K., Eyring, H., The theory of rate processes (Mc Graw-Hill Book Company, Inc. New York and London) 1941.

[156] Gelefand, I. M. and Tsetlin, M. L., Diklady Akad. Nauk SSSR 137 (1961) 2.

[157] Gagarin, S. G. and PolaK, L. S., Kinetika i Kataliz 6 (1969) 181.

[158] Polyanyi, J. C., Acc. Chemical Research 5 (5) (1972) 1961.

[159] CaCciatore, M., CaPitelli, M., J.Q.S.R.T. 16 (1976) 325.

[160] Capitelli, M., Molinari, E., Kinetics of dissociation processes in plasmas in the low and intermediate pressure range, in Topics in Current Chemistry Plasma Chemistry II (ed.) (Springer Verlag) 1982.

[161] PolaK, L. S. et al., Kinetics and Thermodynamics of Chemical Reaction in low Temperature Plasma (Moscou Nauka) 1965.

[162] Zel'Dovich, Ya. B., Rayser, Y., Physics of shocks waves and high temperature hydrodynamic phenomena (Academic Press) 1966.

[163] Duff, R. E. et al., J. Chem. Phys. 31 (1959) 1018.

[164] Ammann, P. R., Timmins, R. S., A.I.Ch.E.J. 12 (1966) 956.

[165] CouderT, J. F. et al. 3e Symposium International de Chimie des Plasmas IUPAC Limoges (1977) G-1-7.

[166] Coudert, J. F., Contribution à l'étude de la synthèse des oxydes d'azote par chalumeau à plasma. Thèse 3e cycle Université de Limoges (1978).

[167] Baronnet, J. M., Contribution à l'étude spectroscopique des plasmas d'azote produits par un générateur à arc soufflé : application de la chimie des plasmas : synthèse des oxydes d'azote. Thèse Doctorat d'Etat. Université de Limoges (1978). 
[168] Baronnet, J. M. et al., J. Chimie Physique 75 (1978) 949.

[169] Coudert, J. F. et al., J. Physique 40C7 (1979) 355.

[170] Fauchais, P., Baronnet, J. M., Pure Applied Chemistry 52 (1980) 1669.

[171] Bourdin, E., Coudert, J. F., Fauchais, P., Plasma Chem. Plasma Proc. 2 (1982) 399.

[172] Rapakoulias, D., Etude des Processus de fixation de l'azote dans un réacteur chimique à plasma hors équilibre. Thèse Université $\mathrm{P}$. et $\mathrm{M}$. Curie Paris (1979).

[173] Amouroux, J., Cavadias, S., Rapakoulias, D., Revue Phys. Appl. 14 (1979) 969.

[174] Botchway, G., Venugopalan, M., Phys. Chimie (Wiesbaden) 121 (1), (1980) 103.

[175] Brooks, B. W., Seafort, R. M., J. Appl. Chem. Biotech. 24 (1974) 621.

[176] Eremin, E. N., Rubatsova, V., Russ. J. Phys. Chem. 74(1) (1973) 356.

[177] Amouroux, J., Bergougnan, M. P., Gicquel, A., Annales de Chimie 8 (1983) 319.

[178] Meubus, P., J. Electroch. Soc. 122(2) (1975) 298.

[179] Revoll, M. F., Thèse, Université Paris VI, Paris 1981.

[180] LeCuILler, M., Thèse Université Paris Sud, 1980.

[181] Polyanyi, J. C., Acc. Chem. Rech. 5 (1972) 161.

[182] Rapakoulias, D., Domange, M., Amouroux, J., Revue Phys. Appl. 16 (1981).

[183] Rapakoulias, D., Amouroux, J., Revue Phys. Appl. 15 (1980) 1251-1259.

[184] Rapakoulias, D., Cavadias, S., Amouroux, J., Revue Phys. Appl. 15 (1980), 1261-1265.

[185] Rapakoulias, D., Amouroux, J., Revue Phys. Appl. 17 (1982) 95.

[186] Mc Creery, J., Wolken, G., J. Chem. Phys. 65 (1976) 1310.

[187] Mc Creery, J., Wolken, G., J. Chem. Phys. 67 (1977) 2551.

[188] Venugopalan, M., Veprek, S., Kinetics and Catalysis in Plasma Chemistry in Topics in Current Chemistry : Plasma Chemistry IV (ed.) (Springer Verlag Berlin) 1983.

[189] Amouroux, J., Cavadias, S., Heating of a fluidized bed by the injection of a thermal plasma. Application to the synthesis of nitrogen oxides. ISPC 6 Montreal july 83 (ed.) Prof. M. Boulos, Univ. de Sherbrooke $\mathrm{CN}$.

[190] Boulos, M. I., IEEE Trans. on Plasma Sc. PS 6 (1978) 93.

[191] Boulos, M. I., Gagne, R. and Barnes, R. M., C.J.Ch. E 58 (1980) 367.

[192] Vardelle, M., Vardelle, A., Besson, J. L., FaUChaIs, P., Revue Phys. App. 16 (1981) 425.

[193] Wilks, P. H., Thorpe, M. L., The heating of solids in high temperature plasma, Paper presented at American Chemical Society, Symposium on High Temperature Chicago (1970).

[194] BonEt, C., Int. J. of Heat Mass Trans. 17 (1974) 743.

[195] Proulx, P., Mostaghimi, J., Boulos, M., Plasma particle interaction effects in induction plasma modelling under dense loading conditions, ISPC 6 , Montreal, july 83 (ed.) Prof. M. Boulos, Univ. de Sherbrooke, CN.

[196] Boulos, M. I., Gauvin, W. H., Can. J. Chem. Eng. 52 (1974) 335.
[197] HaRvey, F. J. et al., A model of particle heat transfer in arc heated gas streamer, in Int. Round Table on Study and Applications of Transport Phenomena in Thermal Plasma, Odeillo, France 12-18 sept. 1975.

[198] Mathieu, Ph., Lebon, G., J. Non-Equilib. Therm. 7 (1982) 129.

[199] Mathieu, M. C., Contribution à l'étude expérimentale et théorique de la sphéroĩdisation de particule d'argile réfractaire dans un four à plasma, Thèse Docteur-Ingénieur, Université de Limoges, 17 juillet (1979).

[200] Borgianni, C., Capitelli, M., Cramarossa, F., Triolo, L., Molinari, E., Combust. Flame 13 (1969) 181.

[201] Capitelli, M., Cramarossa, F., Triolo, L., MoliNARI, E., Combust. Flame 15 (1970) 23.

[202] Bhattacharyya, D., Gauvin, W. H., A.I.Ch.E.J. 21 (1975) 879.

[203] Philips, W. F., Phys. Fluids 18 (1975) 144.

[204] Pruppacher, H. R., Leclair, B. P., Hamielec, A. E., J. Fluid. Mech. 44 (1970) 781.

[205] Lewis, J. A., Gauvin, W. H., A.I.Ch.E. 19 (1973) 982.

[206] Chen, Xi, LeE, Y. C., Pfender, E., The importance of knudsen and evaporation effects on modelling in plasma processing, ISPC 6 , Montreal july 83 (ed.) Prof. M. Boulos, Univ. de Sherbrooke, CN.

[207] Sheer, C., Korman, S., Angier, D. J., CahN, R. P., Arc Vaporization of Refractory powders, 2nd Int. Symp. Electrochem. Soc. Boston (1973).

[208] Yoshida, T., Akashi, K., J. Appl. Phys. 48 (1977) 2252.

[209] Bourdin, E., Vardelle, A., Vardelle, M., Boulos, M. I., FauchaIs, P., Computer modelisation of heat and momentum transfer between a particle and $a$ D. C. Plasma jet, 5th Int. Symp. on Plasma Chemistry, Edinburgh, 8-14 aug. (1981).

[210] Vardelle, M., Vardelle, A., Fauchais, P., BouLOS, M. I., A.I.Ch.E. 29 (1983) 236.

[211] Fiszdon, J. K., Lesinski, J., Accélération et fusion de grains dans un jet de plasma d'argon-hydrogène, International Round Table on Study and Applications of Transport Phenomena in Thermal Plasmas, Odeillo, France, sept. 12-16 (1975).

[212] Fiszdon, J. K., Int. J. Heat Mass Transf. 22 (1979) 749.

[213] Vardelle, M., Vardelle, A., Fauchais, P., Study of the trajectories and temperatures of powders in a d.c. plasma jet-Correlation with alumina sprayed coatings ITSC 83 Thermal Spraying, Essen, BRD, 2nd-6th may (1983) (ed.) German Welding Society, Düssel.

[214] Meyer, T. H., Wolf, C. B., TAYlor, R. F., Ciliberti, D. F., Metal powder vaporization using a plasma torch, ISPC 6, Montreal july 83 (ed.) Prof. M. Boulos, Univ. de Sherbrooke, CN.

[215] Gal-Or, B., J. of Eng. Power 102 (1980) 589.

[216] Sayegh, N. N., Gauvin, W. H., A.I.Ch.E. 25 (1979) 522.

[217] Bourdin, E., Fauchais, P., Boulos, M. I., Int. J. Heat Mass Transfer 26 (4) (1983) 567.

[218] Ranz, W. E., Marshall, W. R., Chem. Eng. Prog. 48 (1952) 141.

[219] Dundas, P. H., Paper 1.2 in Round Table on study and applications of Transport Phenomena in thermal 
plasmas, Odeillo, sept. (1975) éd. Laboratoire des Ultra Réfractaires.

[220] Dinulescu, H., Pfender, E., Wilhelmi, H., Calculation of thermodynamic and transport properties of arc furnace plasmas, in the proceedings (p. 138) of the 5th International Symposium on Plasma Chemistry, Heriot Watt University, Edinburgh 10-14 august (1981).

[221] Chen, Xi, Pfender, E., Plasma Chem. Plasma Proc. 3 (1983) 97.

[222] Chen, Xi, Pfender, E., Plasma Chem. Plasma Proc. 2 (1982) 185.

[223] Bourdin, E., Fauchais, P., Vardelle, A., Rev. Gen. Thermique 253 (1983) 47.

[224] Vardelle, M., Vardelle, A., Besson, J. L., FauChaIs, P., Revue Phys. Appl. 16 (1981) 425.

[225] Vardelle, A., Vardelle, M., Fauchais, P., Plasma Chem. Plasma Process. 2 (3) (1982) 255.

[226] Bonet, C., Thermal plasma processing, Chemical Eng. Prog. USA 7212 (1976) 63

[227] Munz, R. J., The decomposition of molybdenum disulphide in an induction plasma tail flame $\mathrm{Ph} . \mathrm{D}$. Dissertation, Mc Gill Univ. (1974).

[228] Gauvin, W. H., Applications of plasma technology to metallurgical application and chemical processing. Continuing education course. ISPC 6 (ed.) Prof. M. Boulos, Univ. of Sherbrooke, CN.

[229] FauchaIs, P., Revue Phys. Appl. 15 (1980) 1281.

[230] Fauchais, P., J. Four Electrique 7 (1982) 9 ; 9 (1982) $7 ; 10$ (1982) 29.

[231] Fey, M. G., Westinghouse Applied Plasma Systems, Pittsburgh, PA, USA, Plasma torch for industry, Workshop on Industrial Plasma Developments, Sherbrooke july 21-23 1983, paper to be asked the author.

[232] Meyer, T. N., Westinghouse Applied Plasma Systems, Pittsburgh, PA, USA, Design considerations for large plasma systems, Workshop on Industrial Plasma Developments, Sherbrooke july 21231983 , paper to be asked the author.

[233] LABrot, M., Aérospatiale, Saint-Médard-en-Jalles, France " High power plasma torch development ", Workshop on Industrial Plasma Developments, Sherbrooke july 21-23 1983, paper to be asked the author.

[234] Camacho, S. L., Technology Application Services Corp., Raleigh, N. C., USA Plasma arc torches for industrial applications, Workshop on Industrial Plasma Developments, Sherbrooke july 21-23 1983, paper to be asked the author.

[235] Christofides, C., IbBerson, V. J., Processing of hydrocarbons in a thermal RF plasma reactor, ISPC 6 , Montreal july 83 (ed.) Prof. M. Boulos, Univ. of Sherbrooke, CN.

[236] KoVENER, G. S., Use of R. F. plasma for thermal pyrolisis of $\mathrm{CH}_{4}$ and heavy oils, ISPC 6, Montreal july 83 (ed.) Prof. M. Boulos, Univ. of Sherbrooke, CN.

[237] GaUvin, W. H., Spray drying with superheated stream, a novel approach, Rapport interne, Centre de Recherche de Noranda, Pointe-Claire, Québec, Canada (1980).

[238] Амelot, M. P., Spray arying with plasma-heated water vapour., M. Eng. Thesis Dpt of Chemical Engineering, Mc Gill Univ. Montreal (1983).
[239] SANTEN, S., SKF Steel Engineering AB, Hofors, Sweden, Plasma Smelting, Workshop on Industrial Plasma Developments, Sherbrooke july 21231983 , paper to be asked the author.

[240] Fey, M. G. and Mililli, W. J., The application of thermal plasma systems to economical scale ironmaking, 5th International Symposium on Plasma Chemistry, august (1981), Heriot Watt Univ. Edinburgh, Scotland.

[241] Poughis, N. et al., AIME Ironmaking proceedings 38 (1979) 224.

[242] Grosdidier, P., Peat gasification in a plasma-heated cocurrent-reactor, M. Eng. Thesis, Dpt. of Chemical Engineering, Mc Gill Univ., Montreal (1982).

[243] Fauchais, P., Baronnet, J. M., Pure Appl. Chem. 52 (1980) 1669.

[244] Coudert, J. F., Catherinot, A., Baronnet, J. M., FAUCHAIS, P., Laser induced fluorescence measurements in non-equilibrium plasma jets, at atmospheric pressure : $\mathrm{NO}$ synthesis in $\mathrm{Ar}-\mathrm{H}_{2}, \mathrm{O}_{2} \mathrm{Gaz}$ mixtures, ISPEC 6, Montreal july 83 (ed.) M. Boulos, Univ. de Sherbrooke, CN

[245] SChylze, R. A., Chem. Ind. 9 (1968) 1539.

[246] Müller, R., PruCKert, C., Recent developments for the production of acetylene from coal by the Hüls arc process, ISPC 6, Montreal, july 83 (ed.) Prof. M. Boulos, Univ. de Sherbrooke, CN.

[247] Shakourzadeh, K., Amouroux, J., Acetylene black production in a plasma pilot plant, ISPC 6, Montreal, july 83 (ed.) Prof. M. Boulos, Univ. de Sherbrooke, CN.

[248] NeunsChwander, E., J. Less Common Metals 11 (1966) 365.

[249] SAyce, I. G., Pure Appl. Chem. 48 (1976) 215.

[250] SAYCE, I. G., Thermal Syndicate, Tyne and Wear, UK, Ultra-pure glasses via plasma processing, Workshop on Industrial Plasma Developments, Sherbrooke july 21-23 1983, paper to be asked the author.

[251] B.P. 1035191 et B.P. 1112444

[252] Harvey, F. J. et Meyer, T. N., Metallurgical Transactions B 9B (1978) 615.

[253] Matsumoto, O., Miyzzaki, T., High Temp. Sci. 5 (1973) 40

[254] Excell, S. F., Kunn, W. E., Wilks, P. H., Preparation of ultrafine powders of refractory carbides in an arc plasma, Proceedings of the Fine Particles Symposium, Electrochemical Soc. (1973).

[255] Third International Symposium on Plasma Chemistry, University of Limoges, France, july 1977, see Akashi, K. et al. Bourdin E. et al. Matsumoto O. et al.

[256] Perugini, G., Arc Plasma Reactions for Special Ceramics : V. - TIL Production from $\mathrm{TiCl}_{4}$ and $\mathrm{CH}_{4}$ by an Argon-Plasma Furnace in the Presence and Absence of Hydrogen, Proceedings of Second C.E.R.P. International Ceramic Merting on Research Production, oct. 77, Firenze.

[257] Ronsheim, P., Toth, L. E., Mazza, A., Pfender, E., Mitrofanov, B., Arc Plasma Synthesis of Tungsten Carbides, 88th National AICHE Meeting, Philadelphia P.A. june 1980.

[258] Fauchais, P., Bourdin, E., Coudert, J. F., Mc PHerson, R., High Pressure Plasma and their application to Ceramic Technology published in 
Plasma Chemistry-Topics in Current Chemistry (ed.) Venugopalan and S. Veprêk (Springer Verlag, Berlin) 1983.

[259] Heberlin, J. V. R., Lowry, J. F., Meyer, T. N., CiliberTI, D. F., The reduction of tetrachlorosilane by sodium at high temperalure in a laboratory scale experiment, Proc. 4th Int. Symp. on Plasma Chemistry, Univ. of Zurich, Switzerland, aug. (1979), p. 716.

[260] FEY, M., Development of a process for high capacity arc heater production of Si for solar arrays, Contract 954589. Final Technical Report, Westinghouse Electric Corporation, Power Circuit Breaker Div., Trafford Pa, USA.

[261] AsadA, C. et al., Plasma Induction Heating in Third Int. Symp. on Electroslag Melting Process, Pittsburgh USA (1971).

[262] Yamada, H., Yoshida, K., Shimiza, T., Development of plasma arc melting of titanium, Proc. 7th ICVM (1982), Tokyo, Japan.

[263] SChOumakeR, H. R. P., Fours à chauffage plasma dans Int. Round Table on Study and Application of Transport Phenomena in Thermal Plasmas Odeillo CNRS sept. (1975).

[264] Roman, W. C., United Technologies Research Center, East Hartford, Conn, USA Plasma Processing for materials reclamation. Workshop on Industrial Plasma Developments, Sherbrooke, july 21-23 1983, paper to be asked the author.

[265] Magnolo, G., Can. Min. Metall. Bull. 57 (1964) 57.

[266] Collin, P. et Stickler, H., Stahl u Eisen 5 (1980) 220.

[267] Pateyron, B., F.iuchais, P., Experimental study of steel vaporization and aerosol formation, ISPC 6, Montreal july 83 (ed.) Prof. M. Boulos, Univ. de Sherbrooke, $\mathrm{CN}$.

[268] Meyer, T. N., Wolf, C. B., TAYlor, R. F., Ciliberti, D. F., Metal powder vaporisation using a plasma torch, cf. [267].

[269] Barcza, N. A., Mini, K., Randburg, South Africa, Application of Transformed arc plasma to the melting of metal fines. Workshop on Industrial Plasma Developments, Sherbrooke, july 21-23 1982, paper to be asked the author.

[270] MAC RAE, D. R. et al., Ferrovanadium production by plasma carbothermic reduction of vanadium oxide, 3e Sym. Int. de Chimie des Plasmas, Univ. de Limoges, juillet 1977.

[271] Fey, M. G., Harvey, F. J., Plasma heating devices in the electric energy economy, Meetings Eng. Q. May (1976), pp. 27-30.

[272] Pickles, C. A., I.E.E.E.E. Trans 18 (1978) 369.

[273] HARris, V. et al., Arc decomposition of throdonite, J. Electrochem. Soc. 106 (1959) 874-6.

[274] BAYLiss, R. K. and DeRRY, R., High temperature studies in the extraction of beryllium, J. Appl. Chem. 16 (1966) 114-21.

[275] Everest, D. A., Napier, E. and Wells, R. A., Processes for the extraction of beryllium from flotation concentrates of beryl. In High Temperature Refactory Metals, Krinwsky, W.A. ed. (New York : Gordon and Breach) 1978, 113-28 (Metall. Soc. Conf. Proc. 34 pt 1).
[276] Shelley, T. R. and Charles, J. A., Arc electrolysis of complex tin-containing oxide melts, Trans. Inst. Metall. sect. C : Mineral Process, Extr. Metall. 79 (1970) C 259.

[277] BaretT, M. F. et al., Trans. Inst. Min. Metall. 84 (1975) C-231.

[278] Shelley, T. R. and Charles, J. A., Arc electrolysis of lead silicate melts, Trans. Inst. Min. Metall. (Sect. C : Mineral Process. Extr. Metall.) 78 (1969) C-177-80.

[279] Recasans, J., Bortaud, P. and Bonnier, E., Fusion et électrolyse d'oxydes métalliques réfractaires, Rev. Hautes Temp. Réfract. 4 (1967) 281.

[280] S.K.F. Steel Engineering A.D., The Plasma zinc process for recovery of metals from iron oxides and low-grade materials. Rapport interne S.K.F. 14 sept. 1981.

[281] WILKS, P. H. and THORPE, M. L., The heating of solids in high temperature plasma. Paper presented at American Chemical Society, Symposium on high temperature, Chicago 1970, $13 \mathrm{p}$.

[282] FEY, M. et al., Spheroidisation of magnetite using and A.C. arc heater, International Round Table on Study and application of transport phenomena in thermal plasmas, Proceedings, Odeillo sept. 1975, ed. Labo. Ultra Réfractaire.

[283] SChNell, C. R. et al., The industrial application of plasma technology for the production of fumed silica, Symposium on Commercial Potential for Arcs and Plasma Processes, Atlantic City, N.J., 8-11 sept. 1974.

[284] LANDT, U. et al., Production of smouth and spheroidal ferrosilicon particles S.A. Pat. 720850, appl. 1971.

[285] MathieU, M. C., Contribution à l'étude expérimentale et théorique de la sphéroidisation de particules d'argile réfractaire dans un four à plasma, Thèse de Doc. Ing. Université de Limoges, 17 juil. (1979).

[286] Gold, D., Bonet, C., Chauvin, G., Mathieu, A. C., Geirnaert, G., Millet, J., Plasma Chem. Plasma Proc. 1 (1981) 161.

[287] Burox, I. S. et al., Rev. Int. Hautes Temp. Refract. 15 (1978) 201.

[288] ZAAT, J. H., Plasma Spraying, Proceedings of the 9th Int. Thermal Spraying Conf. La Haye 23-29 may 1980.

[289] Rykalin, N. N., Kudinov, V. V., Pure Appl. Chem. 48 (1976) 229.

[290] Besson, J. L., Boch, P., Plasma spraying of ceramics. Int. Round Table Discussion on Special Ceramics for Electronics and Electrical Engineering, Varsovie 8-11 oct. (1978).

[291] Vardelle, A., Fauchais, P., Vardelle, M., Actualité Chimique 10 (1981) 69.

[292] ZAAT, J. H., Ann. Rev. Mater. Sci. 13 (1983) 9.

[293] Apelian, D., Palciwal, M., Smith, R. W., Schilling, W. F., Melting and solidification in plasma spray deposition, Internal Report General Electric R. and D. Schenectady (N.Y.) (1983).

[294] Siemens, P., General Electrical Company, Schenectady, NY, USA, " Rapid Solidification plasma deposition at G.E. " see 231.

[295] Chagnon, P., Fauchais, P., Thermal spraying of ceramics, Ceramics International, accepted for publication. 